\title{
Power, control and coalition formation
}

\author{
Citation for published version (APA):
}

Karos, D. (2013). Power, control and coalition formation. [Doctoral Thesis, Maastricht University]. Maastricht University. https://doi.org/10.26481/dis.20130926dk

Document status and date:

Published: 01/01/2013

DOI:

$10.26481 /$ dis.20130926dk

Document Version:

Publisher's PDF, also known as Version of record

\section{Please check the document version of this publication:}

- A submitted manuscript is the version of the article upon submission and before peer-review. There can be important differences between the submitted version and the official published version of record.

People interested in the research are advised to contact the author for the final version of the publication, or visit the DOI to the publisher's website.

- The final author version and the galley proof are versions of the publication after peer review.

- The final published version features the final layout of the paper including the volume, issue and page numbers.

Link to publication

\footnotetext{
General rights rights.

- You may freely distribute the URL identifying the publication in the public portal. please follow below link for the End User Agreement:

www.umlib.nl/taverne-license

Take down policy

If you believe that this document breaches copyright please contact us at:

repository@maastrichtuniversity.nl

providing details and we will investigate your claim.
}

Copyright and moral rights for the publications made accessible in the public portal are retained by the authors and/or other copyright owners and it is a condition of accessing publications that users recognise and abide by the legal requirements associated with these

- Users may download and print one copy of any publication from the public portal for the purpose of private study or research.

- You may not further distribute the material or use it for any profit-making activity or commercial gain

If the publication is distributed under the terms of Article $25 \mathrm{fa}$ of the Dutch Copyright Act, indicated by the "Taverne" license above, 


\section{Power, Control, and Coalition Formation}

\section{Dissertation}

to obtain the degree of Doctor at Maastricht University, on the authority of the Rector Magnificus Prof. dr. L.L.G. Soete, in accordance with the decision of the Board of Deans, to be defended in public on

Thursday, September 26, 2013, at 12:00 hours

$$
\text { by }
$$

Dominik Karos 


\section{Supervisors:}

Prof. Dr. H.J.M. Peters

Prof. Dr. D. Dimitrov (Saarland University)

\section{Assessment Committee:}

Prof. Dr. A.J. Vermeulen (chairman)

Prof. Dr. F. Bloch (École Polytechnique, ParisTech)

Dr. A.J.A. Storcken 


\section{Acknowledgements}

This thesis is the result of three years' research at Maastricht University and Saarland University. I have to thank both my supervisors at both universities for their great support. Dinko Dimitrov offered weekly feedback on my work and fruitful discussions on a variety of topics. He did not only propose interesting topics to work on, he also encouraged me to present my work outside of my office and provided funds so that I could participate in many conferences from Stony Brook to New Delhi.

The meetings with Hans Peters were always a highlight of the month. Intense discussions, detailed feedback, and the emerging of new ideas and projects were usual results. Often it took me a month - until the next meeting - to work through all these thoughts.

Of course I also thank the three members of the assessment committee, Francis Bloch, Ton Storcken, and Dries Vermeulen, for the time they spent with my work.

The number of people who supported me over the last three years, no matter whether with topic related discussions or a recreating afternoon coffee, is uncountable. I will therefore only group them into two coalitions, namely my colleagues, the guys and girls from the second and third floor of building C3.1 at Saarland University, as well as the people from the Center for the Study of Rationality at the Hebrew University, Jerusalem, where I had a great time, both intellectually and personally.

Last but not least I thank my family not only for keeping my back free but also for their patience in times of deadlines and unfinished proofs.

Dominik Karos

Saarbrücken, July 2013 

to P.P. 



\section{Contents}

1 Introduction $\quad \mathbf{9}$

1.1 Simple Games, Power, and Coalitions . . . . . . . . . . . . . . 9

1.2 Notation and Preliminaries . . . . . . . . . . . . . . . 13

2 Coalition Formation in Apex Games

2.1 Introduction . . . . . . . . . . . . . . . . . . 15

2.2 The Model . . . . . . . . . . . . . . . . . . . . 17

2.2.1 Subgames of Simple Games . . . . . . . . . . . . . . . 17

2.2 .2 Apex Games . . . . . . . . . . . . . . . . . . . . 18

2.2 .3 Power Indices . . . . . . . . . . . . . . . . . . . . . . . . . . . . . . . . . . . . .

2.2.4 Hedonic Coalition Formation Games . . . . . . . . . . . . . 21

2.3 General Results . . . . . . . . . . . . . . . . . . . 22

2.4 Applications . . . . . . . . . . . . . . . . 26

2.4.1 The Shapley-Shubik Index . . . . . . . . . . . . . . . . 26

2.4 .2 The Banzhaf Power Index . . . . . . . . . . . . . . . 28

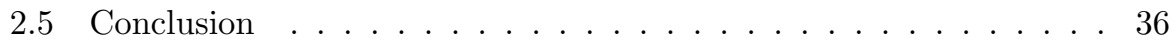

3 Bargaining and Power $\quad 37$

3.1 Introduction . . . . . . . . . . . . . . . . . 37

3.2 The Model . . . . . . . . . . . . . . . . . . . 40

3.3 Coalition Formation . . . . . . . . . . . . . . . . 50

3.4 Infeasible Coalitions . . . . . . . . . . . . . . . . . 60

3.5 Conclusion .......................... 62

4 Mutual Control Structures $\quad 63$

4.1 Introduction . . . . . . . . . . . . . . . 63

4.2 Mutual Control Structures . . . . . . . . . . . . . . . . . 65

4.3 Control Structures and Simple Games . . . . . . . . . . . . . 70

4.4 Power Indices . . . . . . . . . . . . . . . . . . . . . . . . . 74

4.4.1 Independence of the Axioms . . . . . . . . . . . . 84 
4.4.2 Variable number of players . . . . . . . . . . . 86

4.5 Conclusion ........................ 87

$\begin{array}{lr}\text { Bibliography } & 89\end{array}$

$\begin{array}{ll}\text { Index } & 95\end{array}$

$\begin{array}{lr}\text { Summary } & 99\end{array}$

$\begin{array}{ll}\text { About the Author } & 100\end{array}$ 


\section{Chapter 1}

\section{Introduction}

\subsection{Simple Games, Power, and Coalitions}

A committee is a group of players which has to make one or more decisions; think about town councils, parliaments or the UN Security Council. The common characteristic of all committees is that some groups of players, coalitions, can impose their will if they work together. There are not many restrictions how these winning coalitions look like. In a parliament it can be a coalition of parties with the absolute majority of seats, in case of the UN Council a winning coalition must contain 9 votes, including those of all permanent members. In any case, we can describe the situation within a committee by the set of winning coalitions. This structure has already been introduced by von Neumann and Morgenstern (1944) under the name simple game.

Since then mainly two directions of research emerged. One considered simple games as abstract objects, the other placed them into the the context of social choice: A committee has to choose one out of several possible alternatives. Players have preferences over these alternatives and form coalitions according to their interests. Such models as well as conditions on preferences which guarantee a stable outcome of the game, appeared around the same time (see for instance Black, 1948; Arrow, 1951).

We will come back to those ideas later and concentrate first on the consideration of simple games as abstract objects. Neither a set of alternatives nor preferences of players over such alternatives are part of a simple game, that is simple games are independent of any exogenous influences and completely described by the collection of winning coalitions. A game theoretical question is, how power should be allocated to players. An early approach to this question was made by Penrose (1946), who analysed the chances of a player to be part of a win- 
ning coalition or even to be pivotal, i.e., necessary for a coalition to be winning. His ideas have been further developed by Banzhaf (1965) and Coleman (1971). An important result in cooperative game theory in general, and in the theory of simple games in particular, was the introduction of the Shapley value (Shapley, 1953) and the Shapley-Shubik index (Shapley and Shubik, 1954), respectively. Motivated by the question whether a 'consistent criterion for fair representation [of power] can be found', Shapley and Shubik (1954) used the axioms of Shapley (1953) to provide a unique power index.

In a memorandum Shapley (1954) distinguished between three types of solutions of simple games

1. values, where 'the relative power inherent in the different player positions can be defined and measured',

2. coalition structures 'to provide insight into the question of what coalitions structures and what distribution of spoils are most likely to occur', and

3. stable sets, providing "in the simplest cases the "asking price" for each player's participation in a victorious coalition; in other cases "bargaining curves" along which such prices are allowed to vary simultaneously, "discriminatory solutions" in which one or more players are excluded from bargaining, although not necessarily from the payoff, and many other phenomena'.

The variety of solutions has considerably increased since then; not even ten years later Shapley (1962) - which heavily relied on Shapley (1954) - was published and stated the following.

The multiplicity of solution concepts reflects the intrinsic indeterminacy of essential $n$-person games [...] The actual outcomes, in a specific, concrete realization of an abstract game, depend so heavily on exogenous variables that a given solution theory is useful only to the extent that its underlying assumptions represent the conditions under which play takes place.

We will not describe all solution concepts which have been developed between and after those two articles appeared and rather concentrate on those which are related to the theory in this thesis. One important development was the definition of the core of a TU game by Gillies (1959) which is related to the stable set of von Neumann and Morgenstern (1944). Bondareva (1963) and Shapley (1967) characterized those TU games which have a nonempty core. Applied to simple games their result says that the core of a simple game is nonempty if and only if there is at least one player with veto power (as for instance the permanent members of the UN Security Council). The idea of the core is that no group 
of players could improve by splitting from the grand coalition. Aumann and Maschler (1964) argued that players could use their options to bargain about the allocation. The resulting Aumann-Maschler bargaining set is, in contrast to the core, always nonempty. Similar concepts, as the Mas-Colell bargaining set (MasColell, 1989) or the individual stable bargaining set (Greenberg, 1992), appeared later. However, Einy and Wettstein (1996) showed that in case of simple games all three of them coincide with the core if the core is nonempty.

The models mentioned thus far assumed either that the player set would not split into coalitions or that the partition of the player set into coalitions is fixed ex ante. But there have also been models which focused on the formation of coalitions. Both Riker (1962) and de Swaan (1973) give comprehensive overviews over the developments of coalition theory at the respective time. However, most of these models consider simple games in the context of political elections and propose additional assumptions, for instance about the preferences of players (Black, 1948) or their political positions (Axelrod, 1970). Again, we postpone their discussion.

A model of coalition formation in simple games which is independent of exogenous variables was introduced by Peleg (1981). Einy (1985) related Peleg's work to the theory built by Axelrod (1970). Shenoy (1979) used various solutions on simple games to define an abstract (coalition formation) game, again without introducing a set of alternatives or exogenous preferences of players. For a given game he applied a fixed rule on the subgames on winning coalitions (Aumann and Drèze, 1974), which defined payoffs for all players in all winning coalitions. For each player he derived a preference relation over coalitions based on these payoffs, that is coalitions with high payoffs are preferred over coalitions with low payoffs. Based on these preferences a domination relation over partitions of the player set can be derived, which - unfortunately - does not need to be transitive. Shenoy (1979) gave sufficient conditions for the existence of maximal elements with respect to this domination relation, so called core stable partitions; Dimitrov and Haake $(2006,2008 \mathrm{a}, \mathrm{b})$ extended this idea and weakened his condition. Although solutions have previously been defined for cooperative games with coalition structures (see for instance Aumann and Drèze, 1974; Owen, 1977), Shenoy (1979) was the first to consider an endogenous process of coalition formation depending on a fixed rule. Similar approaches for different values have been developed for instance by Hart and Kurz $(1983,1984)$.

Shenoy's abstract games are a subclass of the hedonic coalition formation games introduced by Drèze and Greenberg (1980). In the words of Banerjee et al. (2001), the 'model can be regarded as a reduced two-stage game, where players first form coalitions and next negotiate their payoffs inside coalitions. Once the second step is solved, we are left with the situation described by our hedonic model'. Shenoy (1979) solved 'the second step' by using various solutions 
which have been introduced earlier.

This is where Chapter 2 of this thesis starts. Shenoy (1979) showed that for the hedonic game which is derived from a four player apex game (chief game in von Neumann and Morgenstern, 1944) and the Shapley-Shubik index (Shapley and Shubik, 1954) a core stable partition does not exist. We generalize the class of apex games and apply Shenoy's model on a variety of values with a monotonicity property introduced in Sagonti (1991) and closely related to Young (1985). We give necessary and sufficient conditions on these games for the existence of core stable partitions and fully describe them. Chapter 2 is based on Karos (2012).

In the article of Shenoy (1979) the two lines of theories we mentioned before merged. On one hand the theory of abstract simple games we discussed so far; released from specific contexts, leading to a variety of solutions. On the other hand the combination of a simple game with a set of alternatives, from which players can choose, and preferences of players over these alternatives (in Shenoy's paper the set of alternatives is the set of partitions of the player set, and players' preferences are based on their payoffs in the coalition they belong to). We will now discuss the latter one, that is besides a simple game there is a set of alternatives over which players have preferences and from which the committee has to choose one element. The question in this context is not any more, how power should be allocated to players. It is rather, which coalition should form and which alternative does it choose given player's preferences?

Black (1948) introduced single peakedness and showed that if preferences satisfy this property then there is an alternative such that no winning coalition could form and improve by choosing another one; Dummet and Farquharson (1961) weakened this condition. Greenberg and Weber (1986) defined consecutive games, which are a subclass of the far more general games with non-transferable utilities. Demange (1994) introduced the concept of intermediate preferences over a set of alternatives which also guarantees a stable outcome. Finally, Bogomolnaia and Jackson (2002) worked on hedonic games, compared different properties of preferences, and introduced weaker notions of stability.

Axelrod (1970) developed a model in which political positions of parties in a parliament can be arranged in a unidimensional space and made propositions which coalitions should form. Milchtaich and Winter (2002) skip the assumption of a onedimensional space, the similarity between players is defined by their distance within a (not specified) property space. Similar players try to form coalitions, so the set of possible outcomes is again the set of possible partitions as in Shenoy (1979).

Although there are many further articles on this topic, the few papers mentioned so far, are sufficient to describe the purpose of Chapter 3 of this thesis. We will follow the line of Shenoy (1979) in which players have preferences over partitions, independently of any other set of alternatives. However, in contrast to 
Shenoy (1979) and Banerjee et al. (2001) we do not separate the negotiations inside coalitions from the formation of coalitions. The model will also be extended to situations in which certain coalitions are non-feasible (for instance because of political interests). This can be regarded as a binary version of Milchtaich and Winter (2002), i.e. the distance between two players is either very small, in which case they can form a coalition, or very large, in which case they can't. Chapter 3 is based on Karos (2013).

Another application of simple games, besides parliaments and political councils, can be found in corporate finance. For any corporation the shareholders are players in a simple game: Sufficiently large coalitions of shareholders can enforce strategic decisions the corporation has to make. Gambarelli and Owen (1994) considered the case of a fixed number of firms which are owned by the same set of investors. In Chapter 4, which is based on Karos and Peters (2013), we extend this idea. Instead of separating firms from investors we introduce a model in which companies can mutually control each other. A well known example of corporations which have mutual control over each other are Porsche and VW. Porsche SE has the absolute majority of shares of VW, but is owned (partially) by Porsche $\mathrm{GmbH}$ which in turn belongs to VW. We develop a general game theoretic model to describe these kinds of situations and develop a power index on them based on several axioms.

\section{$1.2 \quad$ Notation and Preliminaries}

Throughout the thesis let $N$ be a finite set of players. A coalition is a subset $S \subseteq N$ and the set $\mathcal{P}=\mathcal{P}(N)$ is the collection of all coalitions. For $i \in N$, let $\mathcal{P}_{i}=\mathcal{P}_{i}(N)$ be the collection of all coalitions which contain $i$. A partition is a set of nonempty coalitions $\pi=\left\{S_{1}, \ldots, S_{m}\right\}$ such that $S_{k} \cap S_{l}=\emptyset$ for all $k \neq l$ and $\bigcup_{k=1}^{m} S_{k}=N$. The collection of all partitions of $N$ is denoted by $\Pi=\Pi(N)$. For a partition $\pi \in \Pi$ and a player $i \in N$, let $\pi(i)$ denote the unique coalition in $\pi$ which contains $i$. For a coalition $S \subseteq N$, let $\Pi_{S}=\Pi_{S}(N)$ be the collection of all partitions containing $S$. A simple game is a function $v: \mathcal{P}(N) \rightarrow\{0,1\}$ with $v(\emptyset)=0$. Particularly, the zero game which assigns 0 to each coalition $S \subseteq N$ is a simple game. A simple game $v$ is called monotonic if $v(S) \leq v(T)$ for all $S, T$ with $S \subseteq T$, and proper if $v(S)+v(N \backslash S) \leq 1$ for all $S \subseteq N$. We denote the set of all monotonic simple games by $\Sigma$ and the set of all proper monotonic simple games by $\Sigma_{p} \subseteq \Sigma$. Given a monotonic simple game $v$, a coalition $S \subseteq N$ with $v(S)=1$ is called winning. A winning coalition $S$ which does not contain any proper subcoalition which is also winning is called minimal winning. If $S$ is a winning coalition and $i \in S$ is such that $v(S \backslash\{i\})=0$ then $i$ is called pivotal in $S$ with respect to $v$. If $i \in N$ is such that $v(S)-v(S \backslash\{i\})=0$ for all coalitions $S$ then $i$ is called a null player (with respect to $v$ ). If $i \in N$ is such 
that $v(S)-v(S \backslash\{i\})=1$ for all winning coalitions $S \subseteq N$ then $i$ is called a veto player (with respect to $v$ ). Two players $i, j \in N$ are called symmetric in $v$ if $v(S \backslash\{i\})=v(S \backslash\{j\})$ for all $S \in \mathcal{P}_{i} \cap \mathcal{P}_{j}$. 


\section{Chapter 2}

\section{Coalition Formation in Apex Games}

\subsection{Introduction}

This chapter considers three concepts of cooperative game theory, namely coalition formation in simple games, apex games, and strongly monotonic solutions. The aim is to find stable outcomes of a hedonic coalition formation game which is derived from a generalized apex game and a strongly monotonic solution.

Given a simple game, an important question is, how powerful various players are. There are different ways how to measure this power. Particularly, it is interesting not only to ask what the power of a player in the overall game is, but also: How powerful is a player within a certain coalition? The power of a player might be used to distribute the worth of a coalition between its members (think of the number of ministries a party gets within a political coalition). Hence, a power measure provides each player with a tool to compare the different coalitions he might belong to. Preferences over coalitions thus derived induce a coalition formation game. The subsequent question can be stated as follows: Which coalition shall form? Or: Which coalition is stable, in the sense that no members will leave it? We assume that the value a player receives in a coalition does not depend on the behaviour of players outside of the coalition, that is that there are no externalities. Such coalition formation games are called hedonic games. They have been introduced by Drèze and Greenberg (1980) and despite the absence of externalities, their analysis is quite complex. Particularly, it is not even clear under which circumstances a stable coalition exists. Although there are some general conditions which guarantee existence (see for instance Banerjee et al., 2001; Bogomolnaia and Jackson, 2002; Iehlé, 2007), an analysis of hedonic games 
which are derived from a simple game and a power measure has not led to sufficiently general results. A good basis of this topic can be found for instance in Dimitrov and Haake (2006, 2008a).

A special subclass of simple games is the class of apex games - our second concept. They have already been studied by von Neumann and Morgenstern (1944). These games with one major player (originally called chief player in von Neumann and Morgenstern (1944), later called apex player) and a set of minor players have been investigated in many articles (see for instance Hart and Kurz, 1983, 1984; Aumann and Myerson, 1988; Montero, 2002).

An apex player can form a winning coalition with each of the minor players. But the set of all minor players together is winning as well. We generalize this idea in the following sense. Let there be several coalitions $I_{k}$ in a simple game and a set $J$ with an empty intersection with each $I_{k}$. A coalition is winning if it contains either at least one coalition $I_{k}$ and at least $r$ players from $J$, or at least $q$ players from $J$, where $q>r$. We call these games general apex games with apex sets $I_{k}$, minor players $J$, apex quota $r$ and minor quota $q$.

General apex games can occur in various situations. A decision process in a company containing two boards (Example 2.2.1) might be considered, as well as the relation between a parliament and a constitutional court (Example 2.2.4), or games with symmetric players who might be bribed (Example 2.4.2).

The third concept we consider is the one of monotonic power indices. Let $u$ and $v$ be two different simple games on the same set of players such that a player is pivotal in all coalitions with respect to $v$ in which he is pivotal with respect to $u$. If there is at least one coalition in which he is pivotal with respect to $v$ but not with respect to $u$ then his power in $v$ should be strictly greater than in $u$. This monotonicity property appears for example in Sagonti (1991), where it is shown to be satisfied by the Shapley-Shubik index (see Shapley, 1953; Shapley and Shubik, 1954), and the Banzhaf index (see Banzhaf, 1965; Coleman, 1971). We show that the normalized Banzhaf index is in general not monotonic in this sense, but that its behaviour on general apex games is quite similar to that of a monotonic power index.

Shenoy (1979) considered hedonic games which are derived from proper monotonic simple games with at most four players together with the Shapley-Shubik index. He showed that the apex game is the only such game for which no stable coalitions exist.

In this chapter we will derive necessary and sufficient conditions for the existence of stable coalitions in hedonic games which are induced by a general apex game and a strongly monotonic power index. In particular, we give necessary and sufficient conditions for the stability of coalitions. We refine these results for the special cases of Shapley-Shubik index, Bazhaf index, and normalized Banzhaf index. Although the existence of a stable coalition in such a hedonic game highly 
depends on the structure of the family of apex sets, it will be shown that many insights can be derived if simple conditions hold true. Particularly, it will be shown that the players of $J$ are an important part of any core stable coalition.

Section 2.2 develops the basics of simple games and hedonic games. We introduce the class of general apex games; and we define power indices as well as some properties they might have, in particular strong monotonicity. Further, we show how to induce a hedonic coalition formation game from a simple game and a power index. In Section 2.3 we state two main results presenting necessary and sufficient conditions for the existence of stable coalitions. Section 2.4 refines the results of Section 2.3 by considering the Shapley-Shubik index, the Banzhaf index, and the normalized Banzhaf index. Section 2.5 briefly summarizes our results and gives some conclusions.

\subsection{The Model}

\subsubsection{Subgames of Simple Games}

Let $v \in \Sigma_{p}$ be a proper monotonic simple game. For each coalition $S$ it can be asked how powerful each $i \in S$ is. Therefore, it is reasonable to consider proper monotonic simple games on coalitions of $N$ (Aumann and Drèze, 1974). For $v \in \Sigma_{p}$ and a set $S \subseteq N$ the subgame $v_{S}$ is defined by

$$
v_{S}(T)=v(S \cap T)
$$

for all $T \subseteq N$. We call $v_{S}$ also the restriction of $v$ on $S$. In particular, $v_{S}$ is a game on $N$. In this new game all players from $N \backslash S$ are null players with respect to $v_{S}$. For each coalition $S$ it is clear that if $v$ is proper then $v_{S}$ is proper; and if $v$ is monotonic then $v_{S}$ is monotonic. So, for any $v \in \Sigma_{p}$ and any $S \subseteq N$ we have $v_{S} \in \Sigma_{p}$. If $S$ is not a winning coalition then $v_{S}$ is just the zero game; if $S=N$ then $v_{S}=v$. As $v_{S} \in \Sigma_{p}$ for each $S \subseteq N$, veto and null players are well defined with respect to $v_{S}$. Given $v$, a veto player in $S$ is a veto player with respect to $v_{S}$ and a null player in $S$ is a null player with respect to $v_{S}$. Particularly, a veto player (null player) $i \in N$ with respect to $v$ is also a veto player (null player) in $v_{S}$ for all winning coalitions $S \in \mathcal{P}_{i}$. We say that $S$ is a null player free coalition if there is no $i \in S$ which is a null player with respect to $v_{S} \cdot{ }^{1}$

If $S \subseteq N$ is winning with respect to $v$ and $S^{\prime} \subseteq S$ contains all $i \in S$ which are not null players with respect to $v_{S}$ then $v_{S}(T)=\bar{v}(S \cap T)=v\left(S^{\prime} \cap T\right)=v_{S^{\prime}}(T)$ for all $T \subseteq N$. Hence, $v_{S}=v_{S^{\prime}}$.

\footnotetext{
${ }^{1}$ In the literature a coalition $S$ is often called null player free if there is no null player with respect to $v$ in $S$ rather than if there is no veto player with respect to $v_{S}$ in $S$ as it is in our paper.
} 


\subsubsection{Apex Games}

Let $N$ be set of players and let $a$ be a proper monotonic simple game on $N$ such that there are $i \in N$ and $J \subseteq N \backslash\{i\}$ with

$$
a(S)= \begin{cases}1, & \text { if } J \subseteq S \text { or }(i \in S \text { and } S \cap J \neq \emptyset) \\ 0, & \text { else. }\end{cases}
$$

Then $a=a_{i J}$ is called an apex game with apex player $i$ and minor players $j \in J$. Apex games have already been introduced by von Neumann and Morgenstern (1944); a broad overview about the existing literature on apex games can be found in Montero (2002). In this subsection we generalize this class of games. The following example provides a motivation for this generalization.

Example 2.2.1. In a stock corporation under German law there is a two-tier board system. An executive board is in charge of managing the company; and a supervisory board has to ensure that the interests of the stakeholders are not violated. Particularly, there are decisions which the supervisory board has to approve. We assume that there is a proper and monotonic voting system in the executive board to make a decision, and such a decision is accepted by the supervisory board if at least $r$ members agree. On the other hand, if at least $q>r$ members of the supervisory board vote for a decision, they are able to enforce the executive board to follow this decision. The following game $v \in \Sigma_{p}$ describes this situation. Let $\left\{I_{k}\right\}$ be the set of those coalitions in the executive board which are able enforce a decision (within the executive board). Let $J$ be the supervisory board. Then

$$
v(S)= \begin{cases}1, & \text { if }(|J \cap S| \geq q) \text { or }\left(\exists I_{k} \in \mathcal{I} \text { with } I_{k} \subseteq S \text { and }|S \cap J| \geq r\right), \\ 0, & \text { else }\end{cases}
$$

The next definition formalizes the idea of Example 2.2.1.

Definition 2.2.2. Let $N$ be a set of players and let $a$ be a simple game on $N$ such that there are

1. $J \subseteq N$ with $|J| \geq 3$,

2. a nonempty collection $\mathcal{I}$ of subsets of $N \backslash J$ with pairwise nonempty intersection such that for all $I^{\prime} \subseteq N \backslash J$ with $I \subseteq I^{\prime}$ for some $I \in \mathcal{I}$ we have $I^{\prime} \in \mathcal{I}$,

3. two integers $0<r<q \leq|J|$ with $q>\frac{1}{2}|J|$ and $r+q \geq|J|+1$, 
such that

$$
a(S)= \begin{cases}1, & \text { if }(|J \cap S| \geq q) \text { or }(\exists I \in \mathcal{I} \text { with } I \subseteq S \text { and }|S \cap J| \geq r) \\ 0, & \text { else. }\end{cases}
$$

Then $a=a_{\mathcal{I} J}^{q, r}$ is a general apex game with apex sets $I \in \mathcal{I}$, minor players $j \in J$, minor quota $q$, and apex quota $r$.

Reconsider Example 2.2.1. The game $v$ is a general apex game: The supervisory board consists of the minor players, each $I_{k}$ is an apex set, $r$ is the apex quota and $q$ is the minor quota.

It can easily be verified that general apex games are proper and monotonic. Particularly, if $r \leq \frac{1}{2}|J|$, it the condition that $I_{1} \cap I_{2} \neq \emptyset$ for all $I_{1}, I_{2} \in \mathcal{I}$ becomes clear. If $r \leq \frac{1}{2}|J|$, there are $J_{1}, J_{2} \subseteq J$ with $\left|J_{1}\right|=\left|J_{2}\right|=r$ and $J_{1} \cap J_{2}=\emptyset$. If there are also $I_{1}, I_{2} \in \mathcal{I}$ such that $I_{1} \cap I_{2}=\emptyset$ then $I_{1} \cup J_{1}$ and $I_{2} \cap J_{2}$ are both winning but have a nonempty intersection. This contradicts properness of $a_{\mathcal{I} J}^{q, r}$. We can say more.

Proposition 2.2.3. Let $\mathcal{I}$ and $J$ satisfy conditions 1 and 2 in Definition 2.2.2 and $q, r$ integers with $0<r<q \leq|J|$. Then $a_{\mathcal{I} J}^{q, r}$ is proper if and only if $q>\frac{1}{2}|J|$ and $r+q \geq|J|+1$.

Proof. We prove the only if part. Assume $q \leq \frac{1}{2}|J|$. Then there is $J^{\prime} \subseteq J$ with $\left|J^{\prime}\right|=q$ and $\left|J \backslash J^{\prime}\right| \geq q$. Hence, $a_{\mathcal{I} J}^{q, r}\left(J^{\prime}\right)=a_{\mathcal{I} J}^{q, r}\left(J \backslash J^{\prime}\right)=1$. Consequently, $a_{\mathcal{I} J}^{q, r}$ is not proper. Assume $r+q \leq|J|$. Then there is $J^{\prime} \subseteq J$ with $\left|J^{\prime}\right|=q$ and $\left|J \backslash J^{\prime}\right| \geq r$. Hence, $a_{\mathcal{I} J}^{q, r}\left(J^{\prime}\right)=a_{\mathcal{I} J}^{q, r}\left(N \backslash J^{\prime}\right)=1$. Therefore, the simple game $a_{\mathcal{I} J}^{q, r}$ is not proper.

Consider the special case with $\mathcal{I}=\{\{i\}\}, r=1$, and $q=|J|$. Then the resulting game is an apex game in the classical sense. We close this section with the following example of a general apex game.

Example 2.2.4. Consider a parliament with 120 members which can pass laws with absolute majority, and which can change the constitution with a majority of two thirds. All members of the parliament have the same voting rights. The constitutional court can forbid a law which is not in line with the constitution (according to the interpretation of the constitutional court). Assume that the parliament would like to pass a law which, however, would be blocked by the constitutional court. Then the parliament could change the constitution such that the controversial law is in line with the new constitution. This situation is a general apex game: $J$ is the set of members of the parliament, $\mathcal{I}$ is the collection of coalitions of judges at the constitutional court which are able to impose a decision, and $r=61, q=81$ are the respective quotas. 


\subsubsection{Power Indices}

A power index is a mapping $\varphi: \Sigma_{p}(N) \rightarrow \mathbb{R}^{N}$. A power index $\varphi$

1. is efficient if $\sum_{i \in N} \varphi_{i}(v)=v(N)$ for all $v \in \Sigma_{p}$;

2. satisfies the null player property if $\varphi_{i}(v)=0$ for all $v \in \Sigma_{p}$ and all null players $i \in N$ with respect to $v$;

3. satisfies the equal treatment property if $\varphi_{i}(v)=\varphi_{j}(v)$ for every game $v \in$ $\Sigma_{p}$ and all $i, j \in N$ which are symmetric with respect to $v$.

These three properties of a power index are standard. All power indices under consideration in this paper satisfy both the null player and the equal treatment property. The first main theorem will not assume efficiency, the second one will strengthen the result under this additional condition.

Let $v \in \Sigma_{p}$ and let $N^{\prime} \subseteq N$ be the coalition of players which are not null players with respect to $v$. As $v_{N^{\prime}}=v$ we have in particular $\varphi_{i}\left(v_{N^{\prime}}\right)=\varphi_{i}(v)$ for all $i \in N$. For this reason we assume throughout the paper that $N$ does not contain any null players with respect to $v$.

The following definition is due to Sagonti (1991), where the monotonicity properties of several power indices are analyzed.

Definition 2.2.5. A power index $\varphi$ is called strongly monotonic if $\varphi_{i}(v)>\varphi_{i}(u)$ for all $i \in N$ and all $u, v \in \Sigma_{p}$ with

$$
\begin{array}{cl}
v(S)-v(S \backslash\{i\}) \geq u(S)-u(S \backslash\{i\}) & \text { for all } S \in \mathcal{P}_{i}, \\
\text { and } \quad v(S)-v(S \backslash\{i\})>u(S)-u(S \backslash\{i\}) & \text { for some } S \in \mathcal{P}_{i} .
\end{array}
$$

This property seems quite natural: If a player is pivotal with respect to $v$ in each coalition $S$ in which he is pivotal with respect to $u$ and in at least one more then he is more powerful in $v$ than in $u$ according to a strongly monotonic power index. A similar definition of strong monotonicity can be found in Young (1985). There, a power index $\varphi$ is called strongly monotonic if $\varphi_{i}(v) \geq \varphi_{i}(u)$ for all players $i \in N$ and all $u, v \in \Sigma_{p}$ which satisfy (2.1). There is a crucial difference between the definitions of Sagonti and Young: If a player $i$ is pivotal in exactly the same coalitions with respect to $u$ and $v$ then Definition 2.2 .5 does not make any statement about the relation between $\varphi_{i}(u)$ and $\varphi_{i}(v)$, whereas Young's notion of monotonicity claims $\varphi_{i}(v)=\varphi_{i}(u)$.

Remark 2.2.6. Let $v \in \Sigma_{p}$, let $\varphi$ be a strongly monotonic power index which satisfies the null player property, and let $i$ in $N$. If $i$ is not a null player with respect to $v$, application of the definition of strong monotonicity on $v$ and the zero game shows that $\varphi_{i}(v)>0$. 


\subsubsection{Hedonic Coalition Formation Games}

A hedonic game is a set $N$ together with a profile of preferences $\left(\succeq_{i}\right)_{i \in N}{ }^{2}$, where $\succeq_{i}$ is defined on $\mathcal{P}_{i}(N)$ for all $i \in N$. Hedonic games belong to a special class of coalition formation games and have been introduced by Drèze and Greenberg (1980). The main characteristic is that for each player $i \in N$, the preference relation $\succeq_{i}$ depends only on the coalitions to which player $i$ belongs, and not on the partiton of $N$. The crucial question is, whether there is a partition of the player set $N$ such that there is no group of players which would leave their coalitions and form a new one together (see Definition 2.2.7). There are several answers to this question; the probably best known examples are sufficient conditions as ordinal balancedness and consecutiveness in Bogomolnaia and Jackson (2002) and the weak top coalition property in Banerjee et al. (2001). Unfortunately, they are not necessary. A characterization of hedonic games for which a stable partition of the player set exists is given in Iehlé (2007). The author defines a property, called pivot balancedness, which is necessary and sufficient for the existence of core stable partitions. Unfortunately, neither can this property efficiently be verified nor is it constructive, in the sense that a core stable partition can easily be found.

Definition 2.2.7. Let $(N, \succeq)$ be a hedonic game and $\pi \in \Pi$. A deviation of $\pi$ is a coalition $S \subseteq N$ such that $S \succ_{i} \pi(i)$ for all $i \in S$. A partition $\pi$ is called core stable if it has no deviations.

A partition $\pi$ is said to be blocked by $S$ if $S$ is a deviation of $\pi$. A partition $\pi$ is said to be blocked if it is blocked by some $S \subseteq N$. The core of a hedonic game $(N, \succeq)$ is the set of all core stable partitions and is denoted by $\mathcal{C}(N, \succeq)$.

Given a proper monotonic simple game $v$ and a power index $\varphi$ we can define a preference profile $\succeq=\left(\succeq_{i}\right)_{i \in N}$ by

$$
S \succeq_{i} T \quad \text { if and only if } \quad \varphi_{i}\left(v_{S}\right) \geq \varphi_{i}\left(v_{T}\right)
$$

for each $S, T \in \mathcal{P}_{i}$. Thus, a proper monotonic simple game $v$, together with a power index $\varphi$, induces a hedonic game. The interpretation of this game is the following: Given a proper monotonic simple game and a power index, each player knows how powerful he is in any coalition $S$. Hence, he prefers a coalition $S$ over $T$ if he has more power in $S$ than in $T$. For convenience we write from here $\varphi_{i}(S)=\varphi_{i}\left(v_{S}\right)$ if the game $v$ is known.

In general, a coalition might be part of one partition which is core stable and part of another one which is not. This is different in case of hedonic games which are induced by a simple game and a power index. Let $v \in \Sigma_{p}$ and let $\varphi$ be a power index which satisfies the null player property. Let $S$ be winning and let

\footnotetext{
${ }^{2} \mathrm{~A}$ preference relation is a complete, transitive, and reflexive binary relation.
} 
$\pi, \sigma \in \Pi_{S}$. Then $\varphi_{i}(\pi(i))=\varphi_{i}(S)=\varphi_{i}(\sigma(i))$ for each $i \in S$. Further $v(T)=0$ for each $T \subseteq N \backslash S$. Hence, for each $j \in N \backslash S$ we have $\varphi_{j}(\pi(j))=\varphi_{j}(\sigma(j))=0$ as $\varphi$ satisfies the null player property. Consequently, either both $\pi$ and $\sigma$ are core stable or none of them is. Since such a family of core stable partitions can be represented by a single coalition, we call a coalition $S \subseteq N$ core stable if each partition $\pi \in \Pi_{S}$ is core stable. We denote the set of core stable coalitions by $\mathcal{C}(N, v, \varphi)$.

\subsection{General Results}

As mentioned before, the question of existence of core stable partitions is difficult to answer. Although we are working with core stable coalitions, the problem is not much easier, as previous works have shown (see for instance Dimitrov and Haake, 2006, 2008a). However, we establish necessary conditions for the existence of core stable coalitions in a hedonic game which is derived from a general apex game together with a strongly monotonic power index.

First we analyze how strong monotonicity affects the power of players within coalitions. The natural intuition is the following: The members of an apex set are more powerful in a coalition $S$ if $S$ contains many minor players, which are substitutable, but not so many that they could form a coalition on their own. For the minor players the situation is similar: As long as there are less than $q$ minor players in a coalition, they are more powerful if there are less other (competing) minor players. This is the statement of Lemma 2.3.1.

Lemma 2.3.1. Let $N$ be a set of players, let $a_{\mathcal{I} J}^{q, r}$ be a general apex game on $N$ with $\mathcal{I}=\left\{I_{k}\right\}_{k=1}^{n}$, and let $\varphi$ be a strongly monotonic power index which satisfies equal treatment. Let $J_{1}, J_{2} \subseteq J$ with $r \leq\left|J_{2}\right|<\left|J_{1}\right|<q$ and let $I \subseteq \bigcup_{k=1}^{n} I_{k}$ be such that $I \cup J_{2}$ is a null player free coalition.

1. For each $i \in I$ we have $\varphi_{i}\left(I \cup J_{1}\right)>\varphi_{i}\left(I \cup J_{2}\right)$.

2. If, in addition, $\varphi$ is efficient and satisfies the null player property then $\sum_{j \in J_{1}} \varphi_{j}\left(I \cup J_{1}\right)<\sum_{j \in J_{2}} \varphi_{j}\left(I \cup J_{2}\right)$.

Proof. Since all $j \in J$ are symmetric with respect to $a_{\mathcal{I} J}^{q, r}$ and since $\varphi$ satisfies the equal treatment property, it can be assumed without loss of generality that $J_{2} \subsetneq J_{1}$. Let $v$ be the restriction of $a_{\mathcal{I} J}^{q, r}$ on $I \cup J_{1}$ and $u$ be the restriction of $a_{\mathcal{I} J}^{q, r}$ on $I \cup J_{2}$.

1. Let $S \subseteq N$ be winning with respect to $u$ and let $i \in I \cap S$ be pivotal in $S$ with respect to $u$. Then $i$ is also pivotal in $S$ with respect to $v$. Hence, $v(S)-v(S \backslash\{i\}) \geq u(S)-u(S \backslash\{i\})$ for all $S \subseteq N$. Let $J^{\prime} \subseteq J$ such that $\left|J^{\prime} \cap J_{2}\right|<r \leq\left|J^{\prime} \cap J_{1}\right|$ and let $S^{\prime}=(S \backslash J) \cup J^{\prime}$. Then $i$ is pivotal in $S^{\prime}$ 
with respect to $v$ but $u\left(S^{\prime}\right)=0$, hence, the inequality is strict. By strong monotonicity of $\varphi$ we have $\varphi_{i}\left(I \cup J_{1}\right)=\varphi_{i}(v)>\varphi_{i}(u)=\varphi_{i}\left(I \cup J_{2}\right)$.

2. By part 1 of the proof $\varphi_{i}\left(I \cup J_{1}\right)>\varphi_{i}\left(I \cup J_{2}\right)$ for each $i \in I$. Hence,

$$
\begin{aligned}
\sum_{j \in J_{1}} \varphi_{j}\left(I \cup J_{1}\right) & =1-\sum_{i \in I} \varphi_{i}\left(I \cup J_{1}\right) \\
& <1-\sum_{i \in I} \varphi_{i}\left(I \cup J_{2}\right)=\sum_{j \in J_{2}} \varphi_{j}\left(I \cup J_{2}\right) .
\end{aligned}
$$

The equalities follow from efficiency and the null player property, and the strict inequality follows from part 1 of the proof.

The condition $\left|J_{1}\right|<q$ in Lemma 2.3.1 is necessary. In fact, if $\left|J_{2}\right|<q$ and $\left|J_{1}\right| \geq q$ then $J_{1}$ is winning in the subgame of $a_{\mathcal{I} J}^{q, r}$ on $I \cup J_{1}$ while $J_{2}$ is not winning in the subgame of $a_{\mathcal{I} J}^{q, r}$ on $I \cup J_{2}$. Therefore, we cannot expect the power of apex players in $I \cup J_{1}$ to be higher than in $I \cup J_{2}$.

We can now prove our first main theorem. This theorem gives a lower bound for the number of minor players which must be contained in a core stable coalition.

Theorem 2.3.2. Let $a_{\mathcal{I} J}^{q, r}$ be a general apex game and let $\varphi$ be a strongly monotonic power index which satisfies equal treatment and the null player property. Then $|S \cap J| \geq \frac{1}{2}|J|$ for each $S \in \mathcal{C}\left(N, a_{\mathcal{I} J}^{q, r}, \varphi\right)$.

Proof. If $r>\frac{1}{2}|J|$ there is nothing to show, so let $r \leq \frac{1}{2}|J|$. Let $S \in$ $\mathcal{C}\left(N, a_{\mathcal{I} J}^{q, r}, \varphi\right)$ and assume that $|S \cap J|<\frac{1}{2}|J|$. Since $S$ must be winning, properness of the game implies that the coalitions $\pi(j)$ are not winning for any $j \in J \backslash S$ and therefore $\varphi_{j}(\pi(j))=0$ for all $\pi \in \Pi_{S}$ and all $j \in J \backslash S$. In particular, $J \backslash S$ is not winning and therefore $|J \backslash S|<q$. Let $I=S \backslash J$. We show that $I \cup(J \backslash S)$ is a deviation of $S$. Indeed, since $I$ is not winning and since all $j \in J \backslash S$ are symmetric with respect to $a_{\mathcal{I} J}^{q, r}$, none of them is a null player in $I \cup(J \backslash S)$. Therefore, $\varphi_{j}(I \cup(J \backslash S))>0$ for all $j \in J \backslash S$ by Remark 2.2.6. Since $\varphi$ satisfies the equal treatment property, and since $|S \cap J|<|J \backslash S|<q$, part 1 of Lemma 2.3.1 applies. Hence, $\varphi_{i}(I \cup(J \backslash S))>\varphi_{i}(S)$ for all $i \in S \backslash J$. Thus, $S$ is blocked.

This result is surprising at first sight: Although the minor players may replace each other in each winning coalition and therefore seem quite weak, each core stable coalition must contain at least half of them.

In the same way as the members of apex sets are more powerful in coalitions with many minor players, the minor players are more powerful in coalitions which contain many apex sets. In particular, it is easy to see in which coalition the power of minor players is maximal. 
Lemma 2.3.3. Let $a_{\mathcal{I} J}^{q, r}$ be a general apex game on a (null player free) set $N$ with $\mathcal{I}=\left\{I_{k}\right\}_{k=1}^{n}$, and let $I^{*}=N \backslash J$. Let $\varphi$ be a strongly monotonic power index. Then $\varphi_{j}\left(I^{*} \cup J^{\prime}\right)>\varphi_{j}\left(I \cup J^{\prime}\right)$ for each $I \subsetneq I^{*}$, each $J^{\prime} \subseteq J$ with $\left|J^{\prime}\right|<q$, and each $j \in J^{\prime}$.

Proof. Let $j \in J$, let $v$ be the apex game restricted to $I^{*} \cup J^{\prime}$ and let $u$ be the apex game restricted to $I \cup J^{\prime}$. If $T \subseteq N$ is such that $j \in T$ is pivotal in $T$ with respect to $u$ then $T \cap\left(I \cup J^{\prime}\right)$ is winning in $a_{\mathcal{I} J}^{q, r}$ and $\left|T \cap J^{\prime}\right|=r$. In this case $T$ is also winning in $v$ due to monotonicity; and $j$ is also pivotal in $T$ with respect to $v$. Let $i \in I^{*} \backslash I$. Since $I^{*}$ does not contain any null players with respect to $a_{\mathcal{I} J}^{q, r}, i$ cannot be a null player with respect to $v$. Hence, there is $T \subseteq N$ such that $i$ and $j$ are pivotal in $T$ with respect to $v$. In particular, $u(T)=0$ and hence, $j$ is not pivotal in $T$ with respect to $u$. Therefore, $\varphi_{j}\left(I^{*} \cup J^{\prime}\right)=\varphi_{j}(v)>\varphi_{j}(u)=\varphi_{j}\left(I \cup J^{\prime}\right)$ due to strong monotonicity of $\varphi$.

If not mentioned otherwise we write $I^{*}=N \backslash J$ and $S^{*}=I^{*} \cup J^{\prime}$ where $J^{\prime} \subseteq J$ with $\left|J^{\prime}\right|=r$ (we do not need to specify $J^{\prime}$ as long as we are working with a power index which satisfies equal treatment). If $\varphi$ is, additionally, strongly monotonic then $\varphi_{j}(S) \leq \varphi_{j}\left(S^{*}\right)$ for all winning coalitions $S$ with $|S \cap J|<q$ and all minor players $j \in S \cap J$ by Lemma 2.3.1 and 2.3.3.

Theorem 2.3.2 holds true without any efficiency assumption. Nevertheless, efficiency is in most cases a desirable property of a power index. The additional assumption of efficiency sharpens the previous result considerably. In part 1 of the following theorem we give a sufficient condition for the nonemptyness of the core and characterize all core stable coalitions if, additionally, $r<\frac{1}{2}|J|$. Part 2 gives a necessary condition for the existence of core stable coalitions and part 3 gives necessary conditions for coalitions to be core stable.

Theorem 2.3.4. Let $a_{\mathcal{I} J}^{q, r}$ be a general apex game with $\mathcal{I}=\left\{I_{k}\right\}_{k=1}^{n}$. Let $\varphi$ be a strongly monotonic power index which satisfies equal treatment, the null player property, and efficiency.

1. If $\varphi_{j}\left(S^{*}\right) \leq \frac{1}{q}$ for all $j \in S^{*} \cap J$ then $\mathcal{C}\left(N, a_{\mathcal{I} J}, \varphi\right) \neq \emptyset$. Suppose, additionally, that $r<\frac{1}{2}|J|$ and let $S \subseteq N$ be null player free. Then $S$ is core stable if and only if $S \subseteq J$ and $|S|=q$.

2. If $\frac{1}{q}<\varphi_{j}\left(S^{*}\right) \leq \frac{1}{2 r}$ for all $j \in S^{*} \cap J$ then $\mathcal{C}\left(N, a_{\mathcal{I} J}, \varphi\right)=\emptyset$.

3. If $\max \left\{\frac{1}{q}, \frac{1}{2 r}\right\}<\varphi_{j}\left(S^{*}\right)$ for all $j \in S^{*} \cap J$ then

$$
\frac{1}{2} \leq \frac{|S \cap J|}{|J|} \leq \frac{|S \cap J|}{q} \leq r \varphi_{j}\left(S^{*}\right)
$$

for each $S \in \mathcal{C}\left(N, a_{\mathcal{I} J}, \varphi\right)$. 
Proof. Let $J^{\prime} \subseteq J$ with $\left|J^{\prime}\right|=q$.

1. Let $\varphi_{j}\left(S^{*}\right) \leq \frac{1}{q}$. We show that $J^{\prime}$ is core stable. $J^{\prime}$ cannot be blocked by any coalition $T$ with $|T \cap J| \geq q$ by efficiency and equal treatment of $\varphi$. By the initial condition $J^{\prime}$ cannot be blocked by $S^{*}$. As $\varphi_{j}(S) \leq \varphi_{j}\left(S^{*}\right)$ for each $S$ with $|S \cap J|<q$ and as each winning coalition must contain at least one minor player, $J^{\prime}$ cannot be blocked at all.

Let now $r<\frac{|J|}{2}$ and let $S$ be core stable. Then $|S \cap J| \geq \frac{1}{2}|J|>r$ by Theorem 2.3.2. If $|S \cap J|>q$ then $S$ is blocked by $J^{\prime}$ due to equal treatment and efficiency. Assume that $|S \cap J|<q$ and let $J^{\prime \prime} \subseteq J$ with $\left|J^{\prime \prime}\right|=r$. From Lemmas 2.3.1 and 2.3.3 follows that

$$
|S \cap J| \cdot \varphi_{j}(S)<r \cdot \varphi_{j}\left(S \backslash J \cup J^{\prime \prime}\right) \leq r \cdot \varphi_{j}\left(S^{*}\right) \leq \frac{r}{q}<\frac{|J|}{2 q} .
$$

Hence, $\varphi_{j}(S)<\frac{|J|}{2 q} \frac{1}{|S \cap J|} \leq \frac{1}{q}$ for all $j \in S \cap J$. So, $S$ is blocked by $J^{\prime}$ and therefore $S$ is not core stable. Let $|S \cap J|=q$. If $S$ contains $i \in S \backslash J$ which is not a null player then $\varphi_{i}(S)>0$ and consequently, $\varphi_{j}(S)<\frac{1}{q}$ for all $j \in S \cap J$ by equal treatment. Thus, $S$ is blocked by $J^{\prime}$. Hence, the only null player free core stable coalitions are those $S \subseteq J$ with $|S|=q$.

2. Let $\frac{1}{q}<\varphi_{j}\left(S^{*}\right) \leq \frac{1}{2 r}$ and let $S \subseteq N$ be winning. First, let $|S \cap J| \geq q$. If there is $i \in S \backslash J$ which is not a null player in $S$ then $S$ is blocked by any $S \cap J$ by equal treatment. If all $i \in S \backslash J$ are null players in $S$ then $S$ is blocked by $S^{*}$, again by equal treatment. Let $|S \cap J|<q$. If $|S \cap J|<\frac{1}{2}|J|$ then $S$ cannot be core stable by Theorem 2.3.2. Hence, let $\frac{1}{2}|J| \leq|S \cap J|<q$. By the initial condition we have $2 r<q \leq|J|$ and therefore $r<|S \cap J|$. With Lemma 2.3 .1 we see $|S \cap J| \cdot \varphi_{j}(S)<r \cdot \varphi_{j}\left(S^{*}\right)$ and therefore

$$
\varphi_{j}(S)<\frac{r}{|S \cap J|} \cdot \varphi_{j}\left(S^{*}\right) \leq \frac{r}{\frac{1}{2}|J|} \cdot \frac{1}{2 r} \leq \frac{1}{|J|} \leq \frac{1}{q} .
$$

Thus, $S$ is blocked by $J^{\prime}$. Therefore, there are no core stable coalitions.

3. Let $\varphi_{j}\left(S^{*}\right)>\frac{1}{2 r}$ and $\varphi_{j}\left(S^{*}\right)>\frac{1}{q}$. Let further $S \subseteq N$ be core stable. As before, Theorem 2.3.2 implies the first inequality. The second inequality is clear. Since $\varphi_{j}\left(J^{\prime}\right)=\frac{1}{q}, J^{\prime}$ is blocked by $S^{*}$. Therefore, each coalition $S$ with $|S \cap J| \geq q$ is either blocked by $J^{\prime}$ or by $S^{*}$. Using Lemma 2.3 .1 as before implies $\varphi_{j}(S) \leq \frac{r}{|S \cap J|} \varphi_{j}\left(S^{*}\right)$. If now $r \varphi_{j}\left(S^{*}\right)<\frac{|S \cap J|}{q}$ then $\varphi_{j}(S)<\frac{1}{q}$. In this case $S$ is blocked by any $J^{\prime}$. Hence, $\frac{|S \cap J|}{q} \leq r \varphi_{j}\left(S^{*}\right)$. 
Note that the cases of Theorem 2.3.4 are exhaustive due to symmetry between minor players. The first two parts relate the power of a minor player in a winning coalition which contains all apex sets to the power he has in a minimal winning coalition containing only minor players. If there is a (null player free) core stable coalition then it must be a minimal winning coalition of minor players; and such a coalition is core stable if and only if it is not blocked by $S^{*}$. The last part of the theorem gives an upper bound for the number of minor players a core stable coalition can contain if minor players are sufficiently powerful in $S^{*}$. In particular, a minimal winning coalition containing only minor players cannot be core stable in this case. However, Theorem 2.3.4 is not a complete if and only if statement: In the third case we give a necessary condition for the existence of core stable coalitions, namely that they cannot be blocked by a minimal winning coalition of minor players. But this does not guarantee that a coalition which satisfies this condition is actually core stable.

\subsection{Applications}

\subsubsection{The Shapley-Shubik Index}

Among the many different power indices the probably best known is the ShapleyShubik index (Shapley, 1953; Shapley and Shubik, 1954), which is defined as

$$
S h_{i}(v)=\sum_{S \in \mathcal{P}_{i}} \frac{(|N|-|S|) !(|S|-1) !}{|N| !}(v(S)-v(S \backslash\{i\}))
$$

for any proper monotonic simple game $v$ and any $i \in N$. It is well known that the Shapley-Shubik index satisfies the conditions of Theorem 2.3.4. But we can strengthen the results presented there for a special subclass of general apex games, namely those with $r=1$. As $r+q \geq|J|+1$ we must have that $q=|J|$. We therefore simply write $a_{\mathcal{I} J}$ for such games and see

$$
a_{\mathcal{I} J}(S)= \begin{cases}1, & \text { if } J \subseteq S \text { or }(\exists I \in \mathcal{I} \text { with } I \subseteq S \text { and } S \cap J \neq \emptyset), \\ 0, & \text { else. }\end{cases}
$$

The next theorem gives a characterization of those general apex games $a_{\mathcal{I} J}$ for which core stable coalitions exist.

Theorem 2.4.1. Let $a_{\mathcal{I} J}$ be a general apex game on $N$ with $\mathcal{I}=\left\{I_{k}\right\}_{k=1}^{n}$ and let $I^{*}=\bigcup_{k=1}^{n} I_{k}$. Then $\mathcal{C}\left(N, a_{\mathcal{I} J}, S h\right) \neq \emptyset$ if and only if $S h_{j}\left(I^{*} \cup\{j\}\right) \leq \frac{1}{|J|}$ for all $j \in J$. Further, if $S \subseteq N$ is core stable and does not contain any null players then $S=J$. 
Proof. As the Shapley-Shubik index satisfies the conditions of Theorem 2.3.4, it is sufficient to show that $S h_{j}\left(I^{*} \cup\{j\}\right) \leq \frac{1}{2 r}=\frac{1}{2}$ for all $j \in J$. In this case the conditions of part 3 of Theorem 2.3.4 are never satisfied. We see that

$$
\begin{aligned}
S h_{j}\left(I^{*} \cup\{j\}\right)= & \sum_{T \subseteq I^{*}} \frac{\left(\left|I^{*}\right|-|T|\right) !|T| !}{\left(\left|I^{*}\right|+1\right) !}\left(a_{\mathcal{I} J}(T \cup\{j\})-a_{\mathcal{I} J}(T)\right) \\
= & \frac{1}{\left|I^{*}\right|+1} \cdot \frac{1}{2}\left[\sum_{T \subseteq I^{*}}\left(\begin{array}{c}
\left|I^{*}\right| \\
|T|
\end{array}\right)^{-1}\left(a_{\mathcal{I} J}(T \cup\{j\})-a_{\mathcal{I} J}(T)\right)\right. \\
& \left.+\sum_{T \subseteq I^{*}}\left(\begin{array}{c}
\left|I^{*}\right| \\
\left|I^{*} \backslash T\right|
\end{array}\right)^{-1}\left(a_{\mathcal{I} J}\left(\left(I^{*} \backslash T\right) \cup\{j\}\right)-a_{\mathcal{I} J}\left(I^{*} \backslash T\right)\right)\right] .
\end{aligned}
$$

The intersection of two apex sets must be nonempty by definition. Let $T^{\prime} \subseteq I^{*}$ such that $T^{\prime} \cup\{j\}$ is winning for all $j \in J$. Then $I^{*} \backslash T^{\prime}$ does not contain any apex set and hence, $I^{*} \backslash T^{\prime} \cup\{j\}$ is not winning. Consequently, $a_{\mathcal{I} J}\left(\left(I^{*} \backslash T^{\prime}\right) \cup\{j\}\right)+$ $a_{\mathcal{I} J}\left(T^{\prime} \cup\{j\}\right) \leq 1$.

Further, for each integer $l \leq\left|I^{*}\right|$ there are $\left(\begin{array}{c}\left|I^{*}\right| \\ l\end{array}\right)$ subsets of $I^{*}$ of size $l$. Altogether

$$
\begin{aligned}
S h_{j}\left(I^{*} \cup\{j\}\right) & \leq \frac{1}{\left|I^{*}\right|+1} \cdot \frac{1}{2} \sum_{T \subseteq I^{*}}\left(\begin{array}{c}
\left|I^{*}\right| \\
|T|
\end{array}\right)^{-1} \cdot 1 \\
& =\frac{1}{\left|I^{*}\right|+1} \cdot \frac{1}{2} \sum_{k=0}^{\left|I^{*}\right|}\left(\begin{array}{c}
\left|I^{*}\right| \\
k
\end{array}\right)\left(\begin{array}{c}
\left|I^{*}\right| \\
k
\end{array}\right)^{-1} \\
& =\frac{1}{2}
\end{aligned}
$$

This completes the proof.

This result is very useful as it is easy to check whether or not core stable coalitions exist. A game $a_{\mathcal{I} J}$ may model the following situations: All minor players have the same information and the coalition $J$ is winning if this information is not leaked. Further, any other coalition is winning only if it has this information. Then only one minor player is needed to pass the information to a coalition. The next example illustrates this idea.

Example 2.4.2. In a city is a drug market on which several dealers are active. These dealers can form coalitions in order to control the drug market and make profit. A coalition of dealers which is able to drive out the remaining players and to control the market on its own is called a syndicate. There cannot be two 
disjoint syndicates, that is, each two syndicates must have at least one member in common. The city's police department wants to dry out the drug market and combats dealers by raids. As long as no policeman leaks out time and place of a raid, the syndicates will not be able to make any profit. However, if a syndicate can bribe at least one of these policemen then the police department is powerless and this syndicate can control the drug market. The situation can be modelled as an apex game $a_{\mathcal{I} J}$, where $\mathcal{I}$ is the collection of syndicates, $J$ is the police force, and $r=1$. If a syndicate $S$ bribes a policeman $j \in J$ then $S \cup\{j\}$ is winning; if $J$ stays together then $J$ wins.

\subsubsection{The Banzhaf Power Index}

Another example of a strongly monotonic power index is the Banzhaf index $\tilde{B} z$ (see Banzhaf, 1965; Coleman, 1971). We follow Owen (1978), where the Banzhaf index of a player $i \in N$ in a game $v \in \Sigma_{p}$ is defined as

$$
\tilde{B} z_{i}(v)=\frac{1}{2^{|N|-1}} \sum_{S \in \mathcal{P}_{i}}(v(S)-v(S \backslash\{i\})) .
$$

It can easily be verified that $\tilde{B} z$ satisfies the null player property and equal treatment on $\Sigma_{p}$. Particularly, Theorem 2.3.2 applies, hence for each general apex game $a_{\mathcal{I} J}^{q, r}$ and each $S \in \mathcal{C}\left(N, a_{\mathcal{I} J}^{q, r}, \tilde{B} z\right)$ it holds that $|S \cap J| \geq \frac{1}{2}|J|$. However, as $\tilde{B} z$ is not efficient, Theorem 2.3.4 does not apply.

Before we come the first theorem of this subsection, we have a closer look on the Banzhaf-Index on general apex games. The next lemma gives an explicit formula for minor players.

Lemma 2.4.3. For any coalition $S \subseteq N$ let $\mathcal{S}^{a}=\{I \subseteq S \backslash J ; I \in \mathcal{I}\}$ be the collection of apex sets which are contained in $S$. Let $a_{\mathcal{I} J}^{q, r}$ be a general apex game on $N$ and let $S \subseteq N$ be a winning coalition. Then

$$
\tilde{B} z_{j}(S)= \begin{cases}\frac{1}{2^{|S|-1}}\left(\left(\begin{array}{c}
|S \cap J|-1 \\
r-1
\end{array}\right)\left|\mathcal{S}^{a}\right|+\left(\begin{array}{c}
|S \cap J|-1 \\
q-1
\end{array}\right)\left(2^{|S \backslash J|}-\left|\mathcal{S}^{a}\right|\right)\right) & \text { if }|S \cap J| \geq q, \\
\frac{1}{2^{|S|-1}}\left(\begin{array}{c}
|S \cap J|-1 \\
r-1
\end{array}\right)\left|\mathcal{S}^{a}\right|, & \text { otherwise }\end{cases}
$$

for each $j \in S \cap J$.

Proof. By definition of $a_{\mathcal{I} J}^{q, r}$ each winning coalition must contain at least $r$ minor players. First, let $S$ be a winning coalition with $|S \cap J| \geq q$ and let $j \in S \cap J$. Let $T \subseteq S$ such that $j$ is pivotal in $T$. Then either $T \backslash J$ does not contain any apex set and $|T \cap J|=q$ or $T$ contains at least one apex set and $|T \cap J|=r$. There are $\left(\begin{array}{c}|S \cap J|-1 \\ q-1\end{array}\right)$ subsets $J^{\prime} \subseteq S \cap J$ of size $q$ which contain $j$ and $\left(\begin{array}{c}|S \cap J|-1 \\ r-1\end{array}\right)$ subsets 
$J^{\prime} \subseteq S \cap J$ of size $r$ which contain $j$. Player $j$ is pivotal in each union of a set of the second type with any set in $\mathcal{S}^{a}$, hence in $\left(\begin{array}{c}|S \cap J|-1 \\ r-1\end{array}\right)\left|\mathcal{S}^{a}\right|$ coalitions. Further $j$ is pivotal in each union of the first type with any subset of $S \backslash J$ which is not contained in $\mathcal{S}^{a}$, hence in $\left(\begin{array}{c}|S \cap J|-1 \\ q-1\end{array}\right)\left(2^{S \backslash J}-\left|\mathcal{S}^{a}\right|\right)$ coalitions. As no coalition can be of both types at the same time, the first part of the formula is proven.

Let now $S$ be winning with $|S \cap J|<q$ and let $j \in S \cap J$. Let $T \subseteq S$ such that $j$ is pivotal in $T$. Then $T \backslash J$ must contain some apex set and $|T \cap J|=q$. The same arguments as before prove the second part of the formula.

The first theorem of this subsection considers the special case of $r>\frac{1}{2}|J|$. This condition is satisfied, for instance, in Example 2.2.4, where a law can pass the parliament if it is accepted by an absolute majority of its members. We show that in this case the grand coalition is core stable.

Theorem 2.4.4. Let $a_{\mathcal{I} J}^{q, r}$ be a general apex game with $r \geq \frac{1}{2}(|J|+1)$. Then $N \in \mathcal{C}\left(N, a_{\mathcal{I} J}^{q, r}, \tilde{B} z\right)$.

Proof. We prove the claims in three steps.

Claim 1 Let $S \subseteq T \subseteq N$ with $S \cap J=T \cap J$ and $|S \cap J| \geq q$. Then $\tilde{B} z_{j}(S) \leq$ $\tilde{B} z_{j}(T)$ for all $j \in S \cap J$.

Proof. Let $I \subseteq S$ be an apex set. Then for each $I^{\prime} \subseteq T \backslash S, I \cup I^{\prime} \subseteq T$ is an apex set. As there are $2^{|T \backslash S|}$ such sets, we have $\left|\mathcal{T}^{a}\right| \geq 2^{|T|-|S|}\left|\mathcal{S}^{a}\right|$. With Lemma 2.4 .3 we find

$$
\begin{aligned}
\tilde{B} z_{j}(S) & =\frac{1}{2^{|S|-1}}\left(\left(\begin{array}{c}
|S \cap J|-1 \\
r-1
\end{array}\right)\left|\mathcal{S}^{a}\right|+\left(\begin{array}{c}
|S \cap J|-1 \\
q-1
\end{array}\right)\left(2^{|S \backslash J|}-\left|\mathcal{S}^{a}\right|\right)\right) \\
& \leq \frac{2^{|S|-|T|}}{2^{|S|-1}}\left(\left(\begin{array}{c}
|S \cap J|-1 \\
r-1
\end{array}\right)\left|\mathcal{T}^{a}\right|+\left(\begin{array}{c}
|S \cap J|-1 \\
q-1
\end{array}\right)\left(2^{|T \backslash J|}-\left|\mathcal{T}^{a}\right|\right)\right) \\
& =\frac{1}{2^{|T|-1}}\left(\left(\begin{array}{c}
|T \cap J|-1 \\
r-1
\end{array}\right)\left|\mathcal{T}^{a}\right|+\left(\begin{array}{c}
|T \cap J|-1 \\
q-1
\end{array}\right)\left(2^{|T \backslash J|}-\left|\mathcal{T}^{a}\right|\right)\right) \\
& =\tilde{B} z_{j}(T) .
\end{aligned}
$$

Claim 2 Let $S \subsetneq N$ such that $S \backslash J=N \backslash J$. Then $\tilde{B} z_{j}(S) \leq \tilde{B} z_{j}(N)$ for all $j \in S \cap J$. 
Proof. First, we see that

$$
\begin{aligned}
\frac{1}{2^{|S|-1}}\left(\begin{array}{c}
|S \cap J|-1 \\
r-1
\end{array}\right)\left|\mathcal{S}^{a}\right| & \leq \frac{1}{2^{|S|-1}} \frac{1}{2^{|J \backslash S|}}\left(\begin{array}{c}
|J|-1 \\
r-1
\end{array}\right)\left|\mathcal{S}^{a}\right| \\
& =\frac{1}{2^{|N|-1}}\left(\begin{array}{c}
|J|-1 \\
r-1
\end{array}\right)\left|\mathcal{S}^{a}\right|
\end{aligned}
$$

where the inequality comes from $r \geq \frac{1}{2}(|J|+1)$. Hence, if $|S \cap J|<q$, the claim is proven with the formula in Lemma 2.4.3. If $|S \cap J| \geq q$ we see

$$
\begin{aligned}
\tilde{B} z_{j}(S) & =\frac{1}{2^{|S|-1}}\left(\left(\begin{array}{c}
|S \cap J|-1 \\
r-1
\end{array}\right)\left|\mathcal{S}^{a}\right|+\left(\begin{array}{c}
|S \cap J|-1 \\
q-1
\end{array}\right)\left(2^{|S \backslash J|}-\left|\mathcal{S}^{a}\right|\right)\right) \\
& \leq \frac{1}{2^{|N|-1}}\left(\begin{array}{c}
|J|-1 \\
r-1
\end{array}\right)\left|\mathcal{S}^{a}\right|+\frac{1}{2^{|S|-1}}\left(\begin{array}{c}
|S \cap J|-1 \\
q-1
\end{array}\right) 2^{|S \backslash J|}-\frac{\left|\mathcal{S}^{a}\right|}{2^{|S|-1}} \\
& <\frac{1}{2^{|N|-1}}\left(\begin{array}{c}
|J|-1 \\
r-1
\end{array}\right)\left|\mathcal{S}^{a}\right|+\frac{1}{2^{|S|-1}} \frac{1}{2^{|J \backslash S|}}\left(\begin{array}{c}
|J|-1 \\
q-1
\end{array}\right) 2^{|N \backslash J|}-\frac{\left|\mathcal{S}^{a}\right|}{2^{|N|-1}} \\
& =\frac{1}{2^{|N|-1}}\left(\begin{array}{c}
|J|-1 \\
r-1
\end{array}\right)\left|\mathcal{S}^{a}\right|+\frac{1}{2^{|N|-1}}\left(\begin{array}{c}
|J|-1 \\
q-1
\end{array}\right) 2^{|N \backslash J|}-\frac{\left|\mathcal{S}^{a}\right|}{2^{|N|-1}} \\
& =\tilde{B} z_{j}(N) .
\end{aligned}
$$

The strict inequality follows from $q>r \geq \frac{1}{2}(|J|+1)$ and $|S \cap J|<|J|$.

Claim $3 \quad N$ is core stable.

Let $S \subseteq N$ be winning. Then

$$
\tilde{B} z_{j}(S) \leq \tilde{B} z_{j}((S \cap J) \cup(N \backslash J))
$$

for all $j \in S \cap J$ either by the first claim (if $|S \cap J| \geq q$ ) or by Lemma 2.3.3 (if $|S \cap J|<q)$. Further,

$$
\tilde{B} z_{j}((S \cap J) \cup(N \backslash J)) \leq \tilde{B} z_{j}((N \cap J) \cup(N \backslash J))=\tilde{B} z_{j}(N)
$$

for all $j \in S \cap J$ by the second claim. Hence, $S$ cannot block $N$.

It is worth mentioning that the result of Theorem 2.4.4 is completely independent of $q$ (as long as $q>r$ and $q \geq|J|-r+1$ are satisfied), i.e. it holds true even if $q<|J|$. Hence, the grand coalition is not blocked by any minimal winning coalition of minor players although they could substitute each other in $N$. However, $N$ need not to be the only core stable coalition as the following example shows. 
Example 2.4.5. Let $I=\{1,2,3\}, J=\{4,5,6\}$, and $\mathcal{I}=\left\{I^{\prime} \subseteq I ;\left|I^{\prime}\right| \geq 2\right\}$. We consider the general apex game $a_{\mathcal{I} J}^{2,3}$. In each coalition $S$ all minor players and all apex players are symmetric, hence $\tilde{B} z(S)$ depends only on $|S \cap J|$ and $|S \cap I|$. The following table gives $\tilde{B} z_{i}$ and $\tilde{B} z_{j}$ for all possible cases, where $i \in S \cap I$ and $j \in S \cap J$.

\begin{tabular}{cccc}
$|S \cap J|$ & $|S \cap I|$ & $\tilde{B} z_{j}(S)$ & $\tilde{B} z_{i}(S)$ \\
\hline 2 & 2 & $\frac{2}{16}$ & $\frac{2}{16}$ \\
2 & 3 & $\frac{4}{16}$ & $\frac{2}{16}$ \\
3 & 0 & $\frac{4}{16}$ & - \\
3 & 1 & $\frac{4}{16}$ & 0 \\
3 & 2 & $\frac{5}{16}$ & $\frac{3}{16}$ \\
3 & 3 & $\frac{6}{16}$ & $\frac{3}{16}$
\end{tabular}

We see that not only $N$ is core stable but also $S$ with $|S \cap I|=2$ and $|S \cap J|=3$.

We come to the second theorem of this subsection which considers again the special case $r=1$. As in Theorem 2.3.4 we first give a sufficient condition for the existence of core stable coalitions and a sufficient condition for coalitions to be core stable. The second part states a necessary condition for nonemptyness of the core.

Theorem 2.4.6. Let $a_{\mathcal{I} J}$ be a general apex game with $r=1, q=|J|$, and $\mathcal{I}=\left\{I_{k}\right\}_{k=1}^{n}$. Let further $I^{*}=\bigcup_{k=1}^{n} I_{k}$.

1. If $|\mathcal{I}| \leq 2^{\left|I^{*}\right|-|J|+1}$ then $S \in \mathcal{C}(N, v, \tilde{B} z)$ for all $S \subseteq N$ with $J \subseteq S$.

2. If $2^{\left|I^{*}\right|-|J|+1}<|\mathcal{I}| \leq 2^{\left|I^{*}\right|-\frac{1}{2}|J|-1}$ then $\mathcal{C}(N, v, \tilde{B} z)=\emptyset$.

Proof. First, we apply Lemma 2.4.3 on a general apex game with $r=1$ and see

$$
\tilde{B} z_{j}(S)= \begin{cases}\frac{1}{2^{|J|-1}}, & \text { if } J \subseteq S \\ \frac{\left|\mathcal{S}^{a}\right|}{2^{|S|-1}}, & \text { otherwise. }\end{cases}
$$

for all $S \subseteq N$ and all $j \in S \cap J$. Consequently, minor players are indifferent between all winning coalitions which contain $J$. Let $j \in J$ and let $S^{*}=I^{*} \cup\{j\}$.

1. Let $|\mathcal{I}| \leq 2^{\left|I^{*}\right|-|J|+1}$. Then

$$
\tilde{B} z_{j}\left(S^{*}\right)=\frac{|\mathcal{I}|}{2^{\left|I^{*}\right|}} \leq \frac{2^{\left|I^{*}\right|-|J|+1}}{2^{\left|I^{*}\right|}}=\frac{1}{2^{|J|-1}} .
$$


Hence, $J$ cannot be blocked by $S^{*}$ and, thus, cannot be blocked by any coalition which does not contain $J$ (Lemma 2.3.1 and 2.3.3). As minor players are indifferent between all coalitions containing $J$, none of these coalitions can be blocked.

2. Let $2^{\left|I^{*}\right|-|J|+1}<|\mathcal{I}| \leq 2^{\left|I^{*}\right|-\frac{1}{2}|J|-1}$ and let $S \subseteq N$. If $J \subseteq S$ then

$$
\tilde{B} z_{j}\left(S^{*}\right)=\frac{|\mathcal{I}|}{2^{\left|I^{*}\right|}}>\frac{2^{\left|I^{*}\right|-|J|+1}}{2^{\left|I^{*}\right|}}=\frac{1}{2^{|J|-1}}=\tilde{B} z_{j}(S)
$$

and $S$ is blocked by $S^{*}$.

Let $J \nsubseteq S$. By Theorem 2.3.2, $|S \cap J| \geq \frac{1}{2}|J|$. Further,

$$
\left|\mathcal{S}^{a}\right| \leq 2^{-\left|I^{*} \backslash S\right|}|\mathcal{I}| \leq 2^{|S \backslash J|-\left|I^{*}\right|}|\mathcal{I}|
$$

Hence,

$$
\begin{aligned}
\tilde{B} z_{j}(S) & =\frac{\left|\mathcal{S}^{a}\right|}{2^{|S \cap J|+|S \backslash J|-1}} \leq \frac{2^{|S \backslash J|-\left|I^{*}\right|} \cdot|\mathcal{I}|}{2^{\frac{1}{2}|J|+|S \backslash J|-1}} \\
& \leq \frac{2^{|S \backslash J|-\left|I^{*}\right|} \cdot 2^{\left|I^{*}\right|-\frac{1}{2}|J|-1}}{2^{\frac{1}{2}|J|+|S \backslash J|-1}}=\frac{1}{2^{|J|}}<\tilde{B} z_{j}(J) .
\end{aligned}
$$

Therefore, $S$ is blocked by $J$.

Although $\tilde{B} z$ is not efficient, there is a natural way to construct an efficient version (see for instance Owen, 1978; Dubey and Shapley, 1979), namely

$$
B z_{i}(v)= \begin{cases}\frac{\mu_{i}(v)}{\sum_{j \in N} \mu_{j}(v)}, & \text { if } \mu_{i} \neq 0 \\ 0, & \text { otherwise }\end{cases}
$$

where $\mu_{i}(v)$ is the number of coalitions $S \subseteq N$ in which player $i$ is pivotal with respect to $v$. $B z$ is called normalized Banzhaf index. As before we use the notation $B z(S)=B z\left(v_{S}\right)$ and $\mu_{i}(S)=\mu_{i}\left(v_{S}\right)$ for all coalitions $S \subseteq N$ and all $i \in N$.

In Sagonti (1991) the question is asked - but not answered - whether or not $B z$ is strongly monotonic. The following example shows that in general it is not, not even on simple games.

Example 2.4.7. Let $A=\{1,2\}, B=\{3,4,5\}$, and $N=A \cup B$. Define $v$ on $N$ as

$$
v(S)= \begin{cases}1, & \text { if } A \subseteq S \text { or }(|S \cap A| \geq 1 \text { and }|S \cap B| \geq 2) \\ 0, & \text { else. }\end{cases}
$$


Let $S=\{1,3,4\}$ and $T=\{1,3,4,5\}$. If $B z$ were strongly monotonic then $B z_{1}(T)>B z_{1}(S)$. But $\mu_{1}(S)=\mu_{3}(S)=\mu_{4}(S)=1$, and on the other hand $\mu_{1}(T)=3$ and $\mu_{3}(T)=\mu_{4}(T)=\mu_{5}(T)=2$. Hence, $B z_{1}(S)=\frac{1}{3}=\frac{3}{9}=$ $B z_{1}(T)$. This implies that $B z$ is not strongly monotonic.

This example shows in particular that $B z$ does not satisfy the conditions of Lemmas 2.3.1 and 2.3.3. However, $B z$ satisfies the conclusions of the respective lemma.

Lemma 2.4.8. Let $N$ be a set of players and let $a_{\mathcal{I} J}^{q, r}$ be a general apex game with $\mathcal{I}=\left\{I_{k}\right\}_{k=1}^{n}$ on $N$. Let $J_{1}, J_{2} \subsetneq J$ with $r \leq\left|J_{2}\right|<\left|J_{1}\right|<q$ and $I \subseteq \bigcup_{k=1}^{n} I_{k}$ be such that $I \cup J_{2}$ is winning. Then

1. $B z_{i}\left(I \cup J_{1}\right)>B z_{i}\left(I \cup J_{2}\right)$ for each $i \in I$ which is not a null player in $I \cup J_{1}$ with respect to $a_{\mathcal{I} J}^{q, r}$.

2. $\sum_{j \in J_{1}} B z_{j}\left(I \cup J_{1}\right)<\sum_{j \in J_{2}} B z_{j}\left(I \cup J_{2}\right)$.

Proof. Let $S=I \cup J_{1}$ and let $\mu(S)=\sum_{i \in S \backslash J} \mu_{i}(S) . j \in J_{1}$ is pivotal in $T \subseteq S$ if and only if $T$ is winning and $\left|T \cap J_{1}\right|=r$. There are exactly $\left|\mathcal{S}^{a}\right| \cdot\left(\begin{array}{c}|J|-1 \\ r-1\end{array}\right)$ such sets containing $j$. For each such set $T$ there are $2^{|J|-\left|J_{1}\right|}$ subsets $U \subseteq J \backslash J_{1}$ such that $j$ is pivotal in $T \cup U$ with respect to the apex game restricted to $S$. Hence, there are $\left|\mathcal{S}^{a}\right| \cdot\left(\begin{array}{c}|J|-1 \\ r-1\end{array}\right) \cdot 2^{|J|-\left|J_{1}\right|}$ different coalitions $T \subseteq S$, such that $j$ is pivotal in $T$ with respect to the restriction of $a_{\mathcal{I} J}$ to $S$.

On the other hand let $i \in I$. Then $i$ is pivotal in $T \subseteq S$ if and only if $T$ is winning and $i$ is pivotal in $\left(T \backslash J_{1}\right) \cup J^{\prime}$ with $J^{\prime} \subseteq J_{1}$ and $\left|J^{\prime}\right|=\geq r$. Hence, there are $\mu_{i}\left(I \cup J^{\prime}\right) \cdot \sum_{k=r}^{m}\left(\begin{array}{c}k \\ \left|J_{1}\right|\end{array}\right)$ different coalitions $T \subseteq S$ such that $i$ is pivotal in $T$ with respect to $a_{\mathcal{I} J}^{q, r}$. For each of theses sets there are $2^{|J|-\left|J_{1}\right|} \operatorname{subsets} U$ of $J \backslash J_{1}$ such that $i$ is pivotal in $T \cup U$. Altogether,

$$
\begin{aligned}
B z_{j}\left(I \cup J_{1}\right) & =\frac{\left|\mathcal{S}^{a}\right| \cdot\left(\begin{array}{c}
\left|J_{1}\right|-1 \\
r-1
\end{array}\right) \cdot 2^{|J|-\left|J_{1}\right|}}{\left|J_{1}\right| \cdot\left|\mathcal{S}^{a}\right| \cdot\left(\begin{array}{c}
\left|J_{1}\right|-1 \\
r-1
\end{array}\right) \cdot 2^{|J|-\left|J_{1}\right|}+\mu\left(I \cup J^{\prime}\right) \cdot \sum_{k=r}^{m}\left(\begin{array}{c}
\left|J_{1}\right| \\
k
\end{array}\right) \cdot 2^{|J|-\left|J_{1}\right|}} \\
& =\frac{\left|\mathcal{S}^{a}\right| \cdot\left(\begin{array}{c}
\left|J_{1}\right|-1 \\
r-1
\end{array}\right)}{\left|J_{1}\right| \cdot\left|\mathcal{S}^{a}\right| \cdot\left(\begin{array}{c}
\left|J_{1}\right|-1 \\
r-1
\end{array}\right)+\mu\left(I \cup J^{\prime}\right) \cdot \sum_{k=r}^{m}\left(\begin{array}{c}
\left|J_{1}\right| \\
k
\end{array}\right)} \\
B z_{i}\left(I \cup J_{1}\right) & =\frac{\mu_{i}\left(I \cup J^{\prime}\right) \cdot \sum_{k=r}^{m}\left(\begin{array}{c}
\left|J_{1}\right| \\
k
\end{array}\right) \cdot 2^{|J|-\left|J_{1}\right|}}{\left|J_{1}\right| \cdot\left|\mathcal{S}^{a}\right| \cdot\left(\begin{array}{c}
\left|J_{1}\right|-1 \\
r-1
\end{array}\right) \cdot 2^{|J|-\left|J_{1}\right|}+\mu\left(I \cup J^{\prime}\right) \cdot \sum_{k=r}^{m}\left(\begin{array}{c}
\left|J_{1}\right| \\
k
\end{array}\right) \cdot 2^{|J|-\left|J_{1}\right|}} \\
& =\frac{\mu_{i}\left(I \cup J^{\prime}\right)}{\left|J_{1}\right| \cdot\left|\mathcal{S}^{a}\right| \cdot x\left(\left|J_{1}\right|\right)+\mu\left(I \cup J^{\prime}\right)}
\end{aligned}
$$

where

$$
x\left(\left|J_{1}\right|\right)=\frac{\left(\begin{array}{c}
\left|J_{1}\right|-1 \\
r-1
\end{array}\right)}{\sum_{k=r}^{\left|J_{1}\right|}\left(\begin{array}{c}
\left|J_{1}\right| \\
k
\end{array}\right)} .
$$


To prove the first part of the Lemma we have to show that $x\left(\left|J_{1}\right|\right)<x\left(\left|J_{2}\right|\right)$ or equivalently $\frac{1}{x\left(\left|J_{1}\right|\right)}>\frac{1}{x\left(\left|J_{2}\right|\right)}$. Let $m=\left|J_{2}\right|$. We find that

$$
\begin{aligned}
\frac{1}{x(m+1)} & =\left(\begin{array}{c}
m \\
r-1
\end{array}\right)^{-1} \sum_{k=r}^{m+1}\left(\begin{array}{c}
m+1 \\
k
\end{array}\right) \\
& =\left(\begin{array}{c}
m \\
r-1
\end{array}\right)^{-1}+\left(\begin{array}{c}
m-1 \\
r-1
\end{array}\right)^{-1} \frac{m-r+1}{m} \sum_{k=r}^{m}\left(\begin{array}{c}
m \\
k
\end{array}\right) \frac{m+1}{m-k+1} \\
& =\left(\begin{array}{c}
m \\
r-1
\end{array}\right)^{-1}+\left(\begin{array}{c}
m-1 \\
r-1
\end{array}\right)^{-1} \sum_{k=r}^{m}\left(\begin{array}{c}
m \\
k
\end{array}\right) \frac{m+1}{m-k+1} \frac{m-r+1}{m} \\
& >\left(\begin{array}{c}
m-1 \\
r-1
\end{array}\right)^{-1} \sum_{k=r}^{m}\left(\begin{array}{c}
m \\
k
\end{array}\right)=\frac{1}{x(m)} .
\end{aligned}
$$

Hence, $\frac{1}{x\left(\left|J_{1}\right|\right)}>\frac{1}{x\left(\left|J_{2}\right|\right)}$. The proof of the second part follows from efficiency and the null player property of $B z$ and is identical to the proof of part 3 of Lemma 2.3.1.

In order to obtain the main theorem of this section we also need an upper bound of $B z_{j}(S)$. In the proof will make use of the following Lemma of Dubey and Shapley (1979).

Lemma 2.4.9. Let $v$ be a (not necessarily proper monotonic) simple game on a player set $N$. Let $\omega$ be the number of winning coalitions with respect to $v$ and let $\nu$ be the number of losing coalitions with respect to $v$. Then

$$
\sum_{i \in N} \mu_{i}(v) \geq \lambda \cdot\left\lfloor|N|-\log _{2}(\lambda)\right\rfloor
$$

where $\lambda=\min (\omega, \nu)$ and $\lfloor x\rfloor$ is the greatest integer $k$ with $k \leq x$.

We can now prove that the power of a minor player is bounded in each coalition.

Lemma 2.4.10. Let $a_{\mathcal{I} J}^{q, r}$ be a general apex game. Then $B z_{j}(S) \leq \frac{1}{r+1}$ for all $j \in S \cap J$.

Proof. If $|S \cap J| \geq q>r$, the claim follows from efficiency and the equal treatment property of $B z$. So, let $|S \cap J|<q$. By part 2 of Lemma 2.4 .8 it is sufficient to show that the inequality holds for each winning coalition $S \subseteq N$ with $|S \cap J|=r$. Hence, let $J^{\prime}=S \cap J$ for such a coalition $S$. Then each $j \in J^{\prime}$ is a veto player in the restriction $v$ of $a_{\mathcal{I} J}^{q, r}$ to $S$. Denote by $\omega(v)$ and $\nu(v)$ the number of winning, losing respectively, coalitions in $v$. Then $\mu_{j}(S)=\omega(v)$. As 
apex sets are pairwise non disjoint, we have $\left|\mathcal{S}^{a}\right| \leq 2^{|S|-r-1}$. As there are $2^{|N|-|S|}$ different subsets of $N \backslash S$, we find

$$
\mu_{j}(S)=\omega(v)=\left|\mathcal{S}^{a}\right| \cdot 2^{|N|-|S|} \leq 2^{|S|-r-1} \cdot 2^{|N|-|S|}=2^{|N|-r-1} \leq \nu(v) .
$$

With equation Corollary 1 from (Dubey and Shapley, 1979, see Lemma 2.4.9 in the appendix) we see

$$
\begin{aligned}
\sum_{i \in S} \mu_{i}(S) & \geq \omega(v) \cdot\left\lfloor|N|-\log _{2}(\omega(v))\right\rfloor \\
& \geq \mu_{j}(S) \cdot\left\lfloor|N|-\log _{2}\left(2^{|N|-r-1}\right)\right\rfloor \\
& =\mu_{j}(S) \cdot\lfloor|N|-|N|+r+1\rfloor \\
& =(r+1) \mu_{j}(S) .
\end{aligned}
$$

Thus, $B z_{j}(S)=\frac{\mu_{j}(S)}{\sum_{i \in S} \mu_{i}(S)} \leq \frac{1}{r+1}$.

The following theorem considers again general apex games with $r=1$ as in the previous subsections.

Theorem 2.4.11. Let $a_{\mathcal{I} J}$ be a general apex game with $\mathcal{I}=\left\{I_{k}\right\}_{k=1}^{n}$. Then $\mathcal{C}\left(N, a_{\mathcal{I} J}, B z\right) \neq \emptyset$ if and only if $B z_{j}(S) \leq \frac{1}{|J|}$ for all $S \subseteq N$ and all $j \in S \cap J$. In this case a null player free coalition $S \subseteq N$ is core stable if and only if $S=J$.

Proof. Let $S \subseteq N$ be winning. If $J \subseteq S$ and $S$ contains $i \in N \backslash J$ which is not a null player in $S$ then $S$ is blocked by $J$.

Let $J \nsubseteq S$ and assume that $S \in \mathcal{C}\left(N, a_{\mathcal{I} J}^{q, r}, B z\right)$. With help of Lemma 2.4.8 we can show that $|S \cap J| \geq \frac{1}{2}|J|$, the proof in analogous to the proof of Theorem 2.3.2. We show that $S$ is blocked by $J$. Let $j^{\prime} \in J \backslash S$. By Lemma 2.4 .8 we have that

$$
\sum_{j \in S \cap J} B z_{j}(S)<B z_{j^{\prime}}\left((S \backslash J) \cup\left\{j^{\prime}\right\}\right) .
$$

Together with Lemma 2.4.10 we see

$$
B z_{j}(S)<\frac{1}{|S \cap J|} B z_{j}\left((S \backslash J) \cup\left\{j^{\prime}\right\}\right) \leq \frac{1}{\frac{1}{2}|J|} \frac{1}{2} \leq \frac{1}{|J|}
$$

for each $j \in S \cap J$. So, $S$ is blocked by $J$. We see that if $S \subseteq N$ is core stable then $J \subseteq S$ and $i \in S \backslash J$ must be a null player. Hence, $J$ is the only candidate for a null player free core stable coalition. Indeed, $J$ is core stable if and only if $B z_{j}(S) \leq \frac{1}{|J|}$ for all $S \subseteq N$. 
We see again, that the key for core stability lies in the set of minor players. Either each minimal winning coalition of minor players is core stable or there is no core stable coalition. We close this section with the following Corollary for apex games in the classical sense, which can immediately be derived from Theorems 2.4.1 and 2.4.11.

Corollary 2.4.12. Let $a_{i J}$ be the apex game with apex player $i$ and minor players $J$. Then $\mathcal{C}\left(N, a_{i J}, S h\right)=\mathcal{C}\left(N, a_{i J}, B z\right)=\emptyset$.

\subsection{Conclusion}

Apex games have already been known since von Neumann and Morgenstern (1944). Coalition formation in apex games has been studied in Hart and Kurz (1984) and Aumann and Myerson (1988), in which power indices are used to endogenize coalition formation. We have generalized this class of games and investigated whether or not core stable coalitions exist.

We have shown that in case of the Shapley-Shubik index and the normalized Banzhaf index, a minimal winning coalition of minor players is the only possible stable outcome. The key property of power indices which leads to our results is strong monotonicity. However, as it becomes clear from the proofs, we only need a weaker monotonicity property; namely monotonicity as stated in the conclusion of Lemma 2.3.1 and satisfied by the normalized Banzhaf index (which is not strongly monotonic). The more players can substitute each other in a game, the more powerful are those players which cannot be substituted.

This very general principle applied on the class of generalized apex games is sufficient for the conclusions of Theorems 2.4.1 and 2.4.11: If there is a null player free core stable coalition (with respect to the Shapley index or the normalized Banzhaf index), it contains only minor players and it is minimal winning. 


\section{Chapter 3}

\section{Bargaining and Power}

\subsection{Introduction}

Consider a committee or a parliament which has to make decisions. Usually, players or parties with similar interests form coalitions which are able to enforce their will. Based on possible coalitions a player might join, one can make statements about his power in the committee or in a coalition he is member of.

The measurement of a member's power in a committee has been the subject of many articles. Famous examples are the Shapley-Shubik power index (SSPI, Shapley and Shubik, 1954) and various versions of the Banzhaf-Coleman power index (BCPI, Banzhaf, 1965). However, these indices per se do not consider players' power in coalitions apart from the whole player set. The same holds true for the values presented in de Clippel (2008) and Dutta et al. (2010) which apply to games with externalities.

Shenoy (1979) introduced the power of players in each coalition based on the Shapley-Shubik index. In particular, the author considered a coalition formation game where players' preferences over coalitions depend on their power in coalitions. This concept has been further developed and generalized by Dimitrov and Haake (2006, 2008a,b). However, these ideas of power within a coalition did not take into account anything outside of this coalition.

The Owen value (OV, Owen, 1977) and the Casajus value (CV, Casajus, 2009) are adaptations of the Shapley-Shubik index which take into account the partition of the player set into coalitions. The first one has been used by Hart and Kurz (1983, 1984) to introduce a similar coalition formation game as Shenoy (1979), but under consideration of the behavior of players outside of a fixed coalition. Although the power of a player therefore depends on other coalitions as well, the power a player has one coalition is completely independent of his power in any 
other coalition.

We interpret power as a payoff of players, for instance in a parliament where a government of several parties has to agree on the allocation of cabinet seats among parties. In this case, a player can use the power in one coalition to claim a certain power in another coalition. In other words: Power in one coalition can be used to bargain about power in other coalitions. We illustrate this idea in the following example.

Example 3.1.1. The German Bundestag currently consists of five parliamentary parties, CDU/CSU (1), FDP (2), SPD (3), Linke (4), and B90/Grüne (5). A coalition has the absolute majority if and only if it contains at least one of the following coalitions: $\{1,2\},\{1,3\},\{1,4\}$, or $\{2,3,4\}$. In the model of Shenoy (1979) there is no stable outcome of this game; in the model of Hart and Kurz (1984) each of these four coalitions is stable.

The current government consists of CDU/CSU and FDP. Under the assumption that parties in the opposition do not collaborate, the above mentioned power indices deliver the following values for the governmental parties.

\begin{tabular}{ccccc}
\hline & SSPI & BCPI & OV & CV \\
\hline CDU & $\frac{1}{2}$ & $\frac{1}{2}$ & $\frac{3}{4}$ & $\frac{2}{3}$ \\
\hline FDP & $\frac{1}{2}$ & $\frac{1}{2}$ & $\frac{1}{4}$ & $\frac{1}{3}$ \\
\hline
\end{tabular}

The idea that these two parties are equally powerful, as SSPI and BCPI suggest, is not very convincing, given that there are two other parties outside of the government which each have the absolute majority together with CDU/CSU. OV distinguishes between the two parties, but if we assume that the remaining parties work together, i.e. if the partition of the player set changes, then we have $O V_{C D U}=O V_{F D P}=\frac{1}{2}$ although CDU has much better chances to find a different party for a government coalition than FDP. The cabinet consists of 16 ministers of which 11 are members of CDU/CSU and 5 belong to FDP. Hence, in this example the outside option value is closest to the actual distribution of power between parties.

The models mentioned thus far always made the assumption that the power of players in coalitions is specified ex ante and leads to a coalition formation game. Nevertheless, in reality power is a result from bargaining: players identify their options in various coalitions use them to renegotiate. Hence, a separation of the coalition formation process from the power distribution does not seem convincing.

Our model brings together these two concepts; the power of a player within a coalition depends on two things:

1. His marginal contribution: A player who is needed in a winning coalition is more powerful than a player who could leave the coalition without effect. 
2. His outside option: A player who is very powerful in another coalition is more powerful than a player who has no other options.

We assume that in each winning coalition a bargaining problem (Nash, 1950) occurs and that the allocation of power in coalitions follows a fixed bargaining rule which takes into account marginal contributions and outside options of all players. After any negotiation, the outside options of players may have changed and lead to a new negotiation. We do not focus on this dynamic process, but on the question whether we can find an allocation which is stable with respect to renegotiation. In this case an application of the bargaining rule would not change the result. We will show in Section 3.2 that under very weak conditions on a bargaining rule such an allocation exists. We also consider a special bargaining rule and show that under some restrictions this stable allocation is even unique.

We can interpret such an allocation as the result of exploratory talks between all groups of parties. As this allocation will not be renegotiated, the preferences of players over coalitions based on this allocation are very robust. In Section 3.3 we give conditions for the existence of a coalition which is both internally stable (i.e. no group of players would leave it to stay alone) and Nash stable (no player would leave the coalition to join another one). We also apply our model to the class of apex games. Chapter 2 considered the coalition formation game after application of the Shapley-Shubik index or the normalized Banzhaf-Coleman index. It has been shown that each coalition contains a group of players which can improve by leaving and joining the players outside of this coalition. In the model we develop in this chapter the existence of a coalition which will not be left by any players is guaranteed for various bargaining rules.

In Section 3.4 we further extend our model. Especially in parliaments not all coalitions which could reach the absolute majority are likely to occur. There are parties which will never collaborate due to their political interests. Milchtaich and Winter (2002) introduced a model on which the distance between players in a property space is used to develop a coalition formation theory. In our case we consider only two cases. Either players in coalition are similar enough to collaborate or they are not. In the latter case we speak of an infeasible coalition. As the stable allocation in our model depends on outside options, we have to ensure that the allocation in an infeasible coalition does not affect the allocation in any other coalition. We show that if each player can chose to stay alone, a stable allocation still exists. In particular, if there is at least one possible winning coalition, then we can find a coalition which is stable as before. We apply our model to the German parliament and compare the results of different bargaining rules with the actual government and the distribution of cabinet seats among them. In Section 3.5 we give some concluding remarks and possible further developments of our model. 


\subsection{The Model}

For $i \in N$ and $S \subseteq N$ the marginal contribution of player $i$ to $S$ in $v$ is defined by

$$
d_{i}^{m}(S)=v(S)-v(S \backslash\{i\}) .
$$

Note, that $d_{i}^{m}(S)$ depends on $v$; we skip $v$ in the notation for convenience, though.

Definition 3.2.1. Let $v$ be a simple game. A power configuration $x=\left(x_{i}\right)_{i \in N}$ for $v$ is a vector of maps $x_{i}: \mathcal{P}_{i} \rightarrow \mathbb{R}$ such that $x_{i}(S)=0$ for all $i \in S$ if $v(S)=0$ and $\sum_{i \in S} x_{i}(S) \leq v(S)$ for all $S \subseteq N$. A power configuration $x$ is called efficient if $\sum_{i \in S} x_{i}(S)=v(S)$ for all $S \subseteq N$, and individually rational if $x_{i}(S) \geq v(\{i\})$ for all $S \in \mathcal{P}_{i}$. The collection of all power configurations for $v$ is denoted by $\Delta(v)$ and the collection of all individually rational power configuration is denoted by $\Delta_{i r}(v)$.

We can think of a power configuration as a set of agreements which clarify in each coalition $S$ how power is distributed in $S$. Let $x$ be a power configuration. The condition $x_{i}(S)=0$ for losing coalitions and all $i \in S$ reflects the idea that a player should not have any power if he is member of a losing coalition. Let $S$ be a winning coalition and let $i \in S$. Player $i$ is contained in many other coalitions (for instance $(N \backslash S) \cup\{i\})$, in particular, each coalition $T \subseteq(N \backslash S) \cup\{i\}$ with $i \in T$ ensures him power $x_{i}(T)$. We define player $i$ 's outside option in $S$ as

$$
d_{i}^{o}(S, x)=\max _{T \subseteq N \backslash S} x_{i}(T \cup\{i\}) .
$$

When the members of $S$ are negotiating on how power within $S$ should be shared, the two values $d_{i}^{m}$ and $d_{i}^{o}$ are crucial for the bargaining position of $i$. The next definition specifies what we mean by bargaining.

Definition 3.2.2. A disagreement point for $S$ is a vector $d=d(S) \in \mathbb{R}^{S}$. A bargaining solution is a map $F$ such that $F(S, v(S), d(S)) \in \mathbb{R}^{S}$,

$$
\sum_{i \in S} F_{i}(S, v(S), d(S)) \leq v(S)
$$

for each proper monotonic simple game $v$, each coalition $S \subseteq N$, and each disagreement point $d$ for $S$; and $F(\{i\}, v(\{i\}), d(\{i\}))=v(\{i\})$.

The triple $(S, v(S), d)$ is called a bargaining problem. It describes exactly the situation discussed above: The players in $S$ negotiate about how to distribute $v(S)$ where the disagreement point $d$ represents their bargaining positions. We have mentioned before that the bargaining position of player $i$ depends on two things, namely the marginal contributions $d_{i}^{m}(S)$ and the outside option $d_{i}^{o}(S, x)$, 
given a power configuration $x$. In our model we assume that disagreement points are convex combinations of $d^{m}$ and $d^{o}$. Henceforth, let

$$
d_{i}(S, x)=\alpha d_{i}^{m}(S)+(1-\alpha) d_{i}^{o}(S, x)
$$

be the disagreement point in the bargaining problem within coalition $S$ where $\alpha \in[0,1]$. It is clear that the outside option $d_{i}^{o}(S, x)$ of a player $i \in S$ can be positive only if $(N \backslash S) \cup\{i\}$ is winning. Because of properness of $v$ this can be the case only if $S \backslash\{i\}$ is losing. Hence, a player $i \in S$ can only have a positive outside option if he is pivotal in $S$. The parameter $\alpha$ specifies how this outside option shall be weighted. Many of the further results do not depend on the choice of $\alpha$. For convenience, we do not mention $\alpha$ in these cases, having in mind that $\alpha \in[0,1]$ is fixed but arbitrary.

We are now facing the following problem: Given any power configuration $x$, we have a set of bargaining problems with disagreement points depending on $x$, hence, players renegotiate their power. After applying a bargaining solution $F$, we end up with a new power configuration which leads to renegotiation, again. We are looking for a power configuration which is stable with respect to renegotiation. The next definition formalizes this idea.

Definition 3.2.3. Let $F$ be a bargaining solution and $v$ be a proper monotonic simple game. A power configuration $x \in \Delta(v)$ is called stable with respect to $F$ if for all winning coalitions $S \subseteq N$ and all $i \in S$ the following holds.

$$
\begin{aligned}
x_{i}(S) & =F_{i}(S, v(S), d(S, x)) \\
d_{i}(S, x) & =\alpha d_{i}^{m}(S)+(1-\alpha) d_{i}^{o}(S, x) \\
d_{i}^{o}(S, x) & =\max _{T \subseteq N \backslash S} x_{i}(T \cup\{i\}) .
\end{aligned}
$$

Note that for all power configurations $x \in \Delta(v)$, all winning $S \subseteq N$ and all $i \in S$ we have that $d_{i}^{o}(S, x) \geq v(\{i\}) \geq 0$, hence, $d_{i}(S, x) \geq 0$. For general bargaining solutions $F$ we cannot assume that a stable payoff configuration exists for all proper monotonic simple games. The aim of the remainder of this section is to find sufficient conditions on $F$ such that a stable power configuration exists.

Remark 3.2.4. In classical bargaining theory we have that $\sum_{i \in S} d_{i}(S) \leq v(S)$ for each bargaining problem. We do not restrict our attention to this case. If the disagreement point is such that it cannot be reached by any allocation of $v(S)$, one usually talks about a bankruptcy problem (see for instance Aumann and Maschler, 1985; Curiel et al., 1987).

We can also interpret our bargaining problems as bargaining problems with claims (Chun and Thomson, 1992). There in a coalition $S$ each player $i$ has a disagreement point he could reach if he does not join the $S$ (which in our case would be $v(\{i\}))$ and a claim point (in our case $d_{i}(S, x)$ ). 
We will not distinguish between bargaining problems, bargaining problems with claims, or bankruptcy problems; henceforth we will talk only about bargaining problems and disagreement points. The following properties a bargaining solution might satisfy account for this and are therefore slightly different from definitions which can be found in literature on bargaining problems.

Definition 3.2.5. A bargaining solution $F$ is called

1. individually rational if we have $F_{i}(S, v(S), d) \geq v(\{i\})$ for all bargaining problems $(S, v(S), d)$ and all $i \in S$.

2. efficient if we have $\sum_{i \in S} F_{i}(S, v(S), d)=v(S)$ for all bargaining problems $(S, v(S), d)$ and all $i \in S$.

3. symmetric if we have $F_{i}(S, v(S), d)=F_{j}(S, v(S), d)$ for all bargaining problems $(S, v(S), d)$ with $d_{i}=d_{j}$.

4. continuous if $F(S, v(S),$.$) is continuous for all coalitions S \subseteq N$ and all proper monotonic simple games $v$.

5. fair if for all bargaining problems $(S, v(S), d)$ there is a player $i \in S$ with $F_{i}(S, v(S), d) \geq d_{i}(S)$ only if $F_{j}(S, v(S), d) \geq d_{j}(S)$ for all players $j \in S$.

Individual rationality does not guarantee that all players are satisfied by their power in the sense that $F_{i}(S, v(S), d) \geq d_{i}$. It rather says that no player should have less power than if he stays alone. Since we do not assume that each player $i$ can receive at least $d_{i}$, fairness ensures that all players are on the same side of $d$ : A player $i$ cannot get more than $d_{i}$ if in the same coalition another player $j$ receives less than $d_{j}$. Efficiency is standard, it can be understood as a normalization such that the distributed power in each winning coalition sums up to 1 . Continuity ensures that a small change in disagreement points cannot cause an arbitrarily large change in the bargaining outcome.

Example 3.2.6. Let $v$ be a proper monotonic simple game.

1. The egalitarian bargaining solution is defined as

$$
E_{i}(S, v(S), d(S))=d_{i}(S)+\frac{1}{|S|}\left(v(S)-\sum_{j \in S} d_{j}(S)\right)
$$

for all $i \in N$. Clearly, $E$ is efficient, fair, and continuous. However, $E$ is not individual rational, as $\sum_{j \in S} d_{j}(S)$ might be very large. 
2. The constrained egalitarian bargaining solution (see for instance Curiel et al., 1987) is defined as

$$
\tilde{E}_{i}(S, v(S), d(S))=\max \left\{d_{i}-\lambda, 0\right\}
$$

for all $i \in N$, where $\lambda$ is such that $\sum_{i \in S} E_{i}(S, v(S), d(S))=v(S)$. $\tilde{E}$ is individual rational, efficient, and continuous. However, $\tilde{E}$ is not fair.

3. The proportional bargaining solution ${ }^{1}$ is defined as

$$
P_{i}(S, v(S), d(S))= \begin{cases}\frac{d_{i}(S)}{\sum_{i \in S} d_{i}(S)} v(S), & \text { if } \sum_{i \in S} d_{i}(S) \neq 0 \\ \frac{1}{|S|} v(S), & \text { if } \sum_{i \in S} d_{i}(S)=0\end{cases}
$$

for all $i \in N$. We see on the first sight that $P$ is individual rational, efficient, and fair. But $P$ is not continuous at $d=0$.

The following theorem focuses on continuous bargaining solutions and gives sufficient conditions for the existence of a stable power configuration. The proportional bargaining solution, which is not continuous, is considered in the next section.

Theorem 3.2.7. Let $F$ be a continuous bargaining solution, let $\alpha \in[0,1]$ be fixed but arbitrary, and $v$ be a proper monotonic simple game.

1. If $F$ is individually rational then there is a stable power configuration $x \in$ $\Delta_{i r}(v)$.

2. If $F$ is fair and efficient then there is a stable power configuration $x \in \Delta(v)$.

Proof. Let $F$ be a bargaining solution and $v$ be a proper monotonic simple game. We define the map $\hat{F}: \Delta(v) \rightarrow \Delta(v)$ as

$$
\hat{F}_{i, S}(x)=F_{i}(S, v(S), d(S, x)) .
$$

A power configuration $x \in \Delta(v)$ is stable with respect to $F$ if and only if $\hat{F}(x)=$ $x$. Hence, we have to show that $\hat{F}$ has a fixed point. Before we show that $\hat{F}$ is in both cases a map from a compact convex set on itself, we show that if $F$ is continuous then $\hat{F}$ is continuous as well. For this purpose we need the following construction. Let $x \in \Delta(v), S \subseteq N$ and $i \in S$. Then let $\mathcal{T}_{i}{ }^{S}(x) \subseteq \mathcal{P}(N \backslash S)$ be such that

$$
x_{i}(T \cup\{i\}) \geq x_{i}\left(T^{\prime} \cup\{i\}\right)
$$

\footnotetext{
${ }^{1}$ This is the proportional solution from bankruptcy games (Curiel et al., 1987); particularly, it is different from the proportional bargaining solution introduced by Kalai (1977)
} 
for all $T \in \mathcal{T}_{i}^{S}(x)$ and all $T^{\prime} \subseteq N \backslash S$. That is, given the power configuration $x, \mathcal{T}_{i}^{S}(x)$ is the collection of optimal coalitions for player $i$ outside of $S$. In particular, we have $d_{i}^{o}(S, x)=x_{i}\left(T_{i}\right)$ for all $T_{i} \in \mathcal{T}_{i}^{S}(x)$. Note that

$$
\hat{F}_{i, S}(x)=F_{i}\left(S, v(S), \alpha d_{i}^{m}(S)+(1-\alpha)\left(x_{i}\left(T_{i}\right)\right)_{i \in S}\right)
$$

for all $T_{i} \in \mathcal{T}_{i}^{S}(x)$. Let now

$$
[x]=\left\{y \in \Delta(v) ; \mathcal{T}_{i}^{S}(x) \cap \mathcal{T}_{i}^{S}(y) \neq \emptyset \text { for all } S \subseteq N, i \in S\right\} .
$$

Then $\hat{F}$ is continuous on $[x]$ for all $x \in \Delta(v)$ as $F$ is continuous. By definition of $\mathcal{T}_{i}^{S}(x)$ it is straightforward that $[x]$ is closed for all $x \in \Delta(v)$. As further $N$ and $\mathcal{P}(N)$ are finite, there can only be a finite number of sets of this type, i.e. there are $x_{1}, \ldots, x_{n}$ such that

$$
\Delta(v)=\bigcup_{k=1}^{n}\left[x_{k}\right] .
$$

As $\hat{F}$ is continuous on $\left[x_{k}\right]$ and $\left[x_{k}\right]$ is closed for all $k=1, \ldots, n, \hat{F}$ is continuous on $\Delta(v)$.

We show now that in both cases of the Theorem there is a compact convex subset of $\Delta(v)$ such that $\hat{F}$ maps this set on itself.

1. Let now $F$ be individual rational. Then we have $F\left(\Delta_{i r}(v)\right) \subseteq \Delta_{i r}(v)$.

2. Let $F$ be fair and efficient. Since $F_{i}(\{i\}, v(\{i\}), d)=v(\{i\}) \geq 0$, we have $d_{i}(S) \geq 0$ for all $S \subseteq N$. Let now

$$
Q=\left\{x \in \Delta(v) ;-(|N|-1) \leq x_{i}(S) \leq 1 \text { for all } S \subseteq N, i \in S\right\}
$$

We show that $\hat{F}(Q) \subseteq Q$. Let therefore $x \in Q$ and $S \subseteq N$. We consider two cases:

(a) Let $F_{i}(S, v(S), d(S, x)) \geq d_{i}(S, x) \geq 0$ for all $i \in S$. Since

$$
\sum_{i \in S} F_{i}(S, v(S), d(S, x)) \leq v(S) \leq 1
$$

we have that $0 \leq F_{i}(S, v(S), d(S, x)) \leq 1$. 
(b) Let $F_{i}(S, v(S), d(S, x)) \leq d_{i}(S, x)$. Then

$$
\begin{aligned}
F_{i}(S, v(S), d(S, x)) & =v(S)-\sum_{j \in S \backslash\{i\}} F_{j}(S, v(S), d(S, x)) \\
& \geq-\sum_{j \in S \backslash\{i\}} d_{j}(S, x) \\
& \geq-\sum_{j \in S \backslash\{i\}} \max _{x \in Q, T \subseteq N \backslash S} x_{j}(T \cup\{i\}) \\
& \geq-(|N|-1) .
\end{aligned}
$$

Hence,

$$
-(|N|-1) \leq F_{i}(S, v(S), d(S, x)) \leq d_{i}(S, x) \leq \max _{i, T} x_{i}(T) \leq 1
$$

So, we have that $\hat{F}(x) \in Q$.

As $\Delta_{i r}(v)$ and $Q$ are both compact and convex, we can apply Brouwer's fixed point theorem. Hence, there is a fixed point $x$ of $\hat{F}$. Particularly, in the first case we have $x \in \Delta_{i r}(v)$.

Theorem 3.2.7 ensures the existence of stable power configurations for continuous bargaining solutions under very weak conditions. Together with Example 3.2.6 it implies the following corollary immediately.

Corollary 3.2.8. Let $v$ be a proper monotonic simple game and let $F=E$ or $F=\tilde{E}$. Then there is a stable payoff configuration $x \in \Delta(v)$ with respect to $F$.

Although the existence of a stable power configuration for all proper monotonic simple games is a strong result, Theorem 3.2.7 does not guarantee uniqueness of the stable power configuration. The next example shows that in general the stable power configuration is not unique.

Example 3.2.9. Let $\alpha=0$ so that $d(S, x)=d^{o}(S, x)$, and let $v$ be the proper monotonic simple game on $N=\{1,2,3,4,5,6\}$ with minimal winning coalitions $\{1,2,3\},\{1,4,5\},\{2,4,6\}$, and $\{3,5,6\}$. A stable power configuration with respect to $P, E$, and $\tilde{E}$ is for instance given by $x_{i}(S)=\frac{v(S)}{|S|}$ for all $S \subseteq N$ and all $i \in S$. However, this is not the only stable power configuration. Let $y$ be defined 
as follows:

$$
y_{i}(S)= \begin{cases}0, & \text { if } v(S)=0 \text { or } i \notin S \\ \frac{1}{|S|}, & \text { if } v(S)=1 \text { and }|S| \geq 5, \\ 1, & \text { if }(S=\{1,2,3,6\} \text { or } S=\{1,4,5,6\}) \text { and } i=1, \\ 1, & \text { if }(S=\{1,2,4,6\} \text { or } S=\{1,3,5,6\}) \text { and } i=6, \\ 1, & \text { if } v(S)=1,|S|=3, i=1, \text { and } 1 \in S, \\ 0, & \text { if } v(S)=0,|S|=3, i \neq 1, \text { and } 1 \in S, \\ 1, & \text { if } v(S)=1,|S|=3, i=6, \text { and } 6 \in S, \\ 0, & \text { if } v(S)=0,|S|=3, i \neq 6, \text { and } 6 \in S .\end{cases}
$$

Then we have $\hat{P}(x)=\hat{E}(x)=\hat{\tilde{E}}(x)=x$, that is $x$ is stable with respect to $P$, $E$, and $\tilde{E}$, too.

Although we cannot guarantee uniqueness of a stable power configuration, we can state some properties it must have.

Lemma 3.2.10. Let $v$ be a proper monotonic simple game, let $F$ be a bargaining solution, and let $x \in \Delta(v)$ be stable with respect to $F$.

1. If $F$ is efficient then $x$ is efficient.

2. If $F$ is individually rational then $x$ is individually rational.

3. If $F$ is symmetric and $i, j$ are symmetric with respect to $v$ then $x^{\prime}$, defined as

$$
\begin{aligned}
& x_{i}^{\prime}(S)= \begin{cases}x_{j}(S), & \text { if } i, j \in S, \\
x_{j}((S \backslash\{i\}) \cup\{j\}), & \text { if } i \in S \text { and } j \notin S,\end{cases} \\
& x_{j}^{\prime}(S)= \begin{cases}x_{i}(S), & \text { if } i, j \in S, \\
x_{i}((S \backslash\{j\}) \cup\{i\}), & \text { if } j \in S \text { and } i \notin S,\end{cases}
\end{aligned}
$$

and $x_{k}^{\prime}(S)=x_{k}(S)$ for all $k \neq i, j$ and all $S \subseteq N$, is stable with respect to $F$ as well.

Proof. The first two parts of the lemma are obvious, we prove only the last part. Let $x \in \Delta(v)$ be stable with respect to $F$ and let $i, j \in N$ by symmetric with respect to $v$. Let $p^{i, j}: N \rightarrow N$ be the permutation defined by

$$
p^{i, j}(k)= \begin{cases}i, & \text { if } k=j, \\ j, & \text { if } k=i, \\ k, & \text { if } k \neq i, j\end{cases}
$$


Then $T \in \mathcal{T}_{k}^{S}(x)$ if and only if $p^{i, j}(T) \in \mathcal{T}_{p^{i, j}(k)}^{p^{i, j}(S)}\left(x^{\prime}\right)$. Hence,

$$
\begin{aligned}
F_{p^{i, j}(k)}\left(p^{i, j}(S), v\left(p^{i, j}(S)\right), d\left(p^{i, j}(S), x^{\prime}\right)\right) & =F_{k}(S, v(S), x(T)) \\
& =x_{k}(S) \\
& =x_{p^{i, j}(k)}^{\prime}(S) .
\end{aligned}
$$

The first two parts of the Lemma need no further explanation. For the last part one has to keep in mind that a stable power configuration need not to be unique. In particular, not every stable power configuration is symmetric, i.e. give the same to symmetric players. Lemma 3.2.10 guarantees that the set of all stable power configuration is symmetric, though. An easy consequence is the following corollary.

Corollary 3.2.11. Let $v$ be a proper monotonic simple game and let $F$ be a symmetric bargaining solution such that there is a unique $x \in \Delta(v)$ which is stable with respect to $F$. Then $x_{i}(S)=x_{j}(S)$ for all $S \subseteq N$ with $i, j \in S$ and $x_{i}(S \cup\{i\})=x_{j}(S \cup\{j\})$ for all $S \subseteq N \backslash\{i, j\}$.

We have already mentioned that the proportional bargaining solution $P$ is not continuous at $d=0$, so that we cannot apply Theorem 3.2.7. Nevertheless, the following lemma already states some properties of stable power configurations with respect to $P$, if they exist. We will later use it to prove existence and even uniqueness under some additional conditions.

Lemma 3.2.12. Let $v$ be a proper monotonic simple game let $x \in \Delta(v)$ be stable with respect to $P$.

1. If $S \subseteq N$ is such that no $i \in S$ is pivotal in $S$ with respect to $v$ then $x_{i}(S)=\frac{v(S)}{|S|}$ for all $i \in S$.

2. If $\alpha>0$ and if $S \subseteq N$ is such that there is at least one player in $S$ who is pivotal in $S$ with respect to $v$ then $x_{j}(S)=0$ for all $j \in S$ which are not pivotal in $S$. If, additionally, there is only one player $i \in S$ who is pivotal in $S$ with respect to $v$ then $x_{i}(S)=1$ and $x_{j}(S)=0$ for all $j \in S \backslash\{i\}$.

\section{Proof.}

1. Since no $i$ is pivotal, we have $d_{i}^{m}(S)=0$ for all $i \in S$. Further, by properness of $v$, we have $v((N \backslash S) \cup\{i\})=0$. Hence, $d_{i}^{o}(S, x)=0$ for all $x \in \Delta(v)$. Thus, $d_{i}(S, x)=0$ for all $x \in \Delta(v)$ and all $i \in S$ and therefore $P_{i}(S, v(S), d(S, x))=\frac{v(S)}{|S|}$. 
2. By the same arguments as in the first part we have that $d_{j}(S, x)=0$ for all $x \in \Delta(v)$ and all $j \in S$ which are not pivotal. As $d_{i}^{m}(S)>0$ for each pivotal player $i \in S$ we have that $d_{i}(S, x)>0$ and thus, $P_{j}(S, v(S) d(S, x))=$ $\frac{0}{\sum_{i \in S} d_{i}(S)}=0$. If $i$ is the only pivotal player, we have $P_{i}(S, v(S) d(S, x))=$ 1 by efficiency of $P$.

Note that the last two results in Lemma 3.2.12 depend on the parameter $\alpha$. For $\alpha=1$ the stable power would be unique and very easy to find: For any winning coalition $S$ let $S^{\prime} \subseteq S$ be the set of players who are pivotal in $S$. Then

$$
x_{i}(S)= \begin{cases}\frac{1}{\left|S^{\prime}\right|}, & \text { if } i \in S^{\prime} \\ 0, & \text { otherwise. }\end{cases}
$$

In the next theorem we show that for strictly positive $\alpha$ a stable power configuration with respect to $P$ always exists. Moreover, we give a lower bound for $\alpha$ such that this stable power configuration is unique.

Theorem 3.2.13. Let $v$ be a proper monotonic simple game.

1. Let $\alpha>0$. Then there is $x \in \Delta(v)$ which is stable with respect to $P$.

2. Let $\alpha \geq \frac{|N|}{|N|+2}$. Then there is a unique $x \in \Delta(v)$ which is stable with respect to $P$.

\section{Proof.}

1. Let $\alpha>0$. It has been shown in Lemma 3.2 .12 that $\hat{P}_{i, S}$ is constant and therefore continuous for all coalitions $S \subseteq N$ which do not contain at least two pivotal players. We show that $\hat{P}_{i, S}$ is also continuous for all coalitions $S \subseteq N$ which contain at least two pivotal players. We see that

$$
\hat{P}_{i, S}(x)=\frac{d_{i}(S) v(S)}{\sum_{j \in S} d_{j}(S)}=\frac{d_{i}(S) v(S)}{\alpha \sum_{j \in S} d_{j}^{m}(S)+(1-\alpha) \sum_{j \in S} d_{j}^{o}(S)} .
$$

As $\sum_{j \in S} d_{j}^{m}(S) \geq 2$ and $\alpha>0$, we have that $\hat{P}$ is continuous for all $x \in \Delta(v)$. Since $\hat{P}\left(\Delta_{i r}(v)\right) \subseteq \Delta_{i r}(v)$, there must be a fixed point of $\hat{P}$ in $\Delta_{i r}(v)$ by Brouwer's fixed point theorem.

2. Let $\alpha \geq \frac{|N|-2}{|N|}$. If $v$ is a proper monotonic simple game such that there is $i \in N$ with $v(\{i\})=1$ then the only stable power configuration with respect to $P$ is

$$
x_{k}(S)= \begin{cases}1, & \text { if } k=i \text { and } i \in S \\ 0, & \text { otherwise }\end{cases}
$$


by Lemma 3.2.12. So let $v$ be such that $v(\{i\})=0$ for all $i \in N$. We show that $\hat{P}$ is a contraction on $\Delta_{i r}(v)$. For this purpose, note that for the partial derivatives of $\hat{P}$ we have

$$
\frac{\partial \hat{P}_{i, S}}{\partial x_{j}(T)}=0
$$

for all $S \subseteq N$ which do not contain at least two pivotal players and for all $S \subseteq N$ and all $i \in S$ which are not pivotal in $S$, for all $T \subseteq N$ and all $j \in T$. We also have $\frac{\partial \hat{P}_{i, N}}{\partial x_{j}(T)}=0$ for all $i \in N$, all $T \subseteq N$ and all $j \in T$ as $N$ does not contain any player with a positive outside option. Let therefore $S \subsetneq N$ contain at least two pivotal players and let $i \in S$ be pivotal. Let further $T_{j}^{S}(x) \in \mathcal{T}_{j}^{S}(x)$ for all $j \in S$. Then we have

$$
\frac{\partial \hat{P}_{i, S}}{\partial x_{j}(T)}= \begin{cases}\frac{(1-\alpha)\left(\alpha \sum_{k \neq i} d_{k}^{m}(S)+(1-\alpha) \sum_{k \neq i} d_{k}^{o}(S)\right)}{\left(\alpha \sum_{k \in S} d_{k}^{m}(S)+(1-\alpha) \sum_{k \in S} d_{k}^{o}(S)\right)^{2}}, & \text { if } i=j, T=T_{i}^{S}(x), \\ -\frac{(1-\alpha)\left(\alpha d_{i}^{m}(S)+(1-\alpha) d_{i}^{o}(S)\right)}{\left(\alpha \sum_{k \in S} d_{k}^{m}(S)+(1-\alpha) \sum_{k \in S} d_{k}^{o}(S)\right)^{2}}, & \text { if } i \neq j, T=T_{j}^{S}(x), \\ 0, & \text { otherwise. }\end{cases}
$$

Hence,

$$
\begin{aligned}
\sum_{j \in N, T \subseteq N}\left|\frac{\partial \hat{P}_{i, S} \mid}{\partial x_{j}(T)}\right| \leq & \frac{1-\alpha}{\alpha \sum_{k \in S} d_{k}^{m}(S)+(1-\alpha) \sum_{k \in S} d_{k}^{o}(S)} \\
& +\frac{(1-\alpha)(|S|-1)\left(\alpha d_{i}^{m}(S)+(1-\alpha) d_{i}^{o}(S)\right)}{\left(\alpha \sum_{k \in S} d_{k}^{m}(S)+(1-\alpha) \sum_{k \in S} d_{k}^{o}(S)\right)^{2}} \\
= & \frac{1-\alpha}{\alpha \sum_{k \in S} d_{k}^{m}(S)+(1-\alpha) \sum_{k \in S} d_{k}^{o}(S)} \\
& +\frac{(1-\alpha)|S|\left(\alpha d_{i}^{m}(S)+(1-\alpha) d_{i}^{o}(S)\right)}{\left(\alpha \sum_{k \in S} d_{k}^{m}(S)+(1-\alpha) \sum_{k \in S} d_{k}^{o}(S)\right)^{2}} \\
\leq & \frac{(1-\alpha)\left(\alpha d_{i}^{m}(S)+(1-\alpha) d_{i}^{o}(S)\right)}{\alpha \sum_{k \in S} d_{k}^{m}(S)+(1-\alpha) \sum_{k \in S} d_{k}^{o}(S)} \\
\leq \quad \frac{(1-\alpha)(|S|+1)}{\left(\alpha \sum_{k \in S} d_{k}^{m}(S)+(1-\alpha) \sum_{k \in S} d_{k}^{o}(S)\right)^{2}} & \left.\frac{1-\alpha}{\mid 2 \alpha} d_{k}^{o}(S)\right)^{2}
\end{aligned}
$$


For the Jacobian matrix $D_{\hat{P}}$ we therefore find

$$
\begin{aligned}
\left\|D_{\hat{P}}\right\|_{\infty} & =\max _{i \in N, S \subsetneq N} \sum_{j \in N, T \subseteq N}\left|\frac{\partial \hat{P}_{i, S}}{\partial x_{j}(T)}\right| \\
& \leq \frac{(1-\alpha)|N|}{2 \alpha}-\frac{1-\alpha}{(|N|-1)^{2}}
\end{aligned}
$$

Since this bound is decreasing in $\alpha$ and since $\alpha \geq \frac{|N|}{|N|+2}$ we find

$$
\left\|D_{\hat{P}}\right\|_{\infty} \leq 1-\frac{2}{(|N|+2)(|N|-1)^{2}} .
$$

As the matrix norm $\|\cdot\|_{\infty}$ is compatible with the vector norm $\|\cdot\|_{\infty}$ we have that

$$
\begin{aligned}
\|\hat{P}(x)-\hat{P}(y)\|_{\infty} & \leq\left\|D_{\hat{P}}\right\|_{\infty}\|x-y\|_{\infty} \\
& \leq\left(1-\frac{2}{(|N|+2)(|N|-1)^{2}}\right)\|x-y\|_{\infty}
\end{aligned}
$$

for all $x, y \in \Delta_{i r}(v)$. Hence, $\hat{P}$ is a contraction on $\Delta_{i r}(v)$ and has therefore a unique fixed point by Banach's fixed point theorem.

\subsection{Coalition Formation}

Recall that a hedonic coalition formation game (Drèze and Greenberg, 1980) is a set $N$ together with a profile of preferences $\left(\succeq_{i}\right)_{i \in N}$. For $i \in N$ and $S, T \in \mathcal{P}_{i}$ let $\succeq_{i}$ be defined by

$$
S \succeq_{i} T \quad \text { if and only if } \quad x_{i}(S) \geq x_{i}(T) .
$$

The outcome of a hedonic coalition formation game is a partition of the player set. In our case we are interested in coalitions rather than partitions (see the discussion at the end of Subsection 2.2.4). Therefore we slightly adapt the classical definitions of stability, for the original versions see for instance Bogomolnaia and Jackson (2002).

Definition 3.3.1. Let $v$ be a proper monotonic simple game, $x \in \Delta(v)$, and $S \subseteq N$ be winning. $S$ is called Nash stable (with respect to $x$ ) if for each $i \in S$ 
and each $T \subseteq N \backslash S$ it holds that $x_{i}(T \cup\{i\}) \leq x_{i}(S) .^{2} S$ is called individually stable (with respect to $x$ ) if for each $i \in S$ and each $T \subseteq N \backslash S$ it holds that either $x_{i}(T \cup\{i\}) \leq x_{i}(S)$ or there is $j \in T$ with $x_{j}(T \cup\{i\})<0$.

Roughly speaking, a winning coalition $S$ is Nash stable if no player $i \in S$ has an incentive to leave $S$ and join any coalition $T \subseteq N \backslash S$. The coalition $S$ is individually stable if it is Nash stable or if for each player $i$ who would like to change his coalition from $S$ to $T$, there is at least one player $j \in T$ who would not agree as he would be negatively affected by player $i$ 's move. Clearly, Nash stability implies individual stability; if $x \in \Delta_{i r}(v)$ the definitions are even equivalent.

Let $x \in \Delta_{i r}(v)$. It is easy to see that a winning coalition $S$ which does not contain any pivotal player must be Nash stable as no player can improve by moving to a losing coalition. However, these coalitions do not always seem credible in the following sense: Although no player can improve by leaving the coalition, there might still be a group of players $T$ inside of $S$ which could improve by excluding the remaining players. This motivates the following definition.

Definition 3.3.2. A coalition $S \subseteq N$ is called internally stable (with respect to $x)$ if for each $T \subseteq S$ there is $i \in T$ such that $x_{i}(S) \geq x_{i}(T)$.

The question is now: Can we find a coalition which is both Nash stable and internally stable? The answer is yes, in the following set up.

Theorem 3.3.3. Let $v$ be a proper monotonic simple game, let $F$ be an individually rational and fair bargaining solution, let $\alpha=0$, and let $x \in \Delta(v)$ be stable with respect to $F$. Then there is a coalition $S \subseteq N$ which is both Nash stable and internally stable.

Proof. First we show that there is an internally stable winning coalition $S$. For this purpose note that $x_{i}(N) \geq d_{i}^{o}(N)=0$ for all $i \in N$ by individual rationality. Let now $S_{0}=N$ and $S_{k} \subsetneq S_{k-1}$ such that $x_{i}\left(S_{k}\right)>x_{i}\left(S_{k-1}\right)$ for all $i \in S_{k}$. If $k$ is such that there is no $S_{k+1}$ then $S=S_{k}$ is internally stable. As $N$ is finite, such $S$ must exist.

We show that there is a Nash stable and internally stable coalition. For this reason let $S$ be internally stable. Since $F$ is fair and $\alpha=0$, we have either $x_{i}(S) \geq d_{i}^{o}(S, x)$ for all $i \in S$ or $x_{i}(S)<d_{i}^{o}(S)$ for all $i \in S$. In the first case this means

$$
x_{i}(S) \geq \max _{T \subseteq N \backslash S} x_{i}(T \cup\{i\}),
$$

hence, $S$ is Nash stable. So, let $i \in S$ and let $x_{i}(S)<d_{i}^{o}(S)$. Let $T_{1} \in \mathcal{T}_{i}{ }^{S}(x)$. Since $d_{i}^{o}(S)>x_{i}(S) \geq 0, T_{1}$ must be a winning coalition. Because of individual

\footnotetext{
${ }^{2}$ Note that we do not forbid the existence of a player $j \in N \backslash S$ who could improve by joining S. See also Remark 3.3.7.
} 
rationality there is no losing $T^{\prime} \subsetneq T_{1} \cup\{i\}$ with $x_{j}\left(T^{\prime}\right)>x_{j}\left(T_{1} \cup\{i\}\right)$ for all $j \in T^{\prime}$. As $i$ is pivotal in $T_{1}, i$ is contained in each winning $T^{\prime} \subseteq T_{1} \cup\{i\}$. Since $T_{1} \in \mathcal{T}_{i}^{S}(x)$, we have that $x_{i}\left(T_{1} \cup\{i\}\right) \geq x_{i}\left(T^{\prime}\right)$. Thus, $T_{1}$ is internally stable. Now, either $x_{i}\left(T_{1}\right) \geq d_{i}^{o}\left(T_{1}, x\right)$ or $x_{i}\left(T_{1}\right)<d_{i}^{o}\left(T_{1}, x\right)$. In the first case $T_{1}$ is Nash stable as fairness implies $x_{j}\left(T_{1}\right) \geq d_{j}^{o}\left(T_{1}, x\right)$ for all $j \in T_{1}$. In the latter case we define

$$
T_{k} \in \mathcal{T}_{i}^{T_{k-1}}(x)
$$

for all $k \geq 2$. Then all $T_{k}$ are internally stable and $T_{k}$ is Nash stable if and only if $x_{i}\left(T_{k}\right) \geq x_{i}\left(T_{k+1}\right)$. As $N$ and therefore $\mathcal{P}(N)$ are finite, there is $k$ such that $T_{k+1}=T_{l}$ for some $l \leq k$. Let $k^{*}$ be the first such $k^{*}$. Then

$$
x_{i}\left(T_{k^{*}}\right) \geq x_{i}\left(T_{l}\right)=x_{i}\left(T_{k^{*}+1}\right)=d_{i}^{T_{k^{*}}}
$$

and we see that $T_{k^{*}}$ is Nash stable.

The remainder of this section is devoted to the class of apex games. Recall that an apex game $a_{i J}$ on a player set $N=\{i\} \cup J$, where $|J| \geq 3$, is defined by

$$
a_{i J}(S)= \begin{cases}1, & \text { if }(i \in S \text { and } S \cap J \neq \emptyset) \text { or } J \subseteq S, \\ 0, & \text { otherwise. }\end{cases}
$$

We will show that for each apex game there are unique stable power configuration with respect to the bargaining solutions $E, \tilde{E}$, and $P$. We also investigate the induced hedonic coalition formation game. We already know that we can find internally and Nash stable coalitions in case of $\alpha=0$. Now, we consider arbitrary $\alpha \in[0,1]$ and show under which conditions we can find coalitions which satisfy core stability. We adapt Definition 2.2.7 to our situation and obtain the following definition.

Definition 3.3.4. Let $v$ be a proper monotonic simple game, let $x \in \Delta(v)$, and let $S \subseteq N$ be winning. A deviation of $S$ is a coalition $T$ such that $x_{i}(T)>x_{i}(S)$ for all $i \in S \cap T$ and $x_{i}(T)>0$ for each $i \in T \backslash S$. $S$ is called core stable (with respect to $x$ ) if there is no deviation of $S$.

So, $T$ is a deviation of $S$ if each player in $T$ prefers that $T$ forms over the formation of $S$ : Those players contained in both coalitions have more power in $T$ than in $S$; and those which are only contained in $T$ are powerless if $S$ forms but have positive power in $T$. Recall that if $T$ is a deviation of $S$, we also say that $T$ blocks $S$.

We already know that there is a stable power configuration with respect to the proportional solution if $\alpha>0$. In case of apex games such a power configuration exists also for $\alpha=0$. Moreover this power configuration is even unique for arbitrary $\alpha$, as the following theorem shows. 
Theorem 3.3.5. Let $a_{i J}$ be an apex game. The unique $x \in \Delta\left(a_{i J}\right)$ which is stable with respect to $P$, is given by

$$
\begin{aligned}
& x_{i}(S)= \begin{cases}\frac{1}{|N|}, & \text { if } S=N, \\
\frac{|J|}{(1+\alpha)|J|+1-\alpha}, & \text { if }|S \cap J|=1, \\
1, & \text { if } 2 \leq|S \cap J| \leq|J|-1\end{cases} \\
& x_{j}(S)= \begin{cases}\frac{1}{|N|}, & \text { if } S=N, \\
\frac{1}{|J|}, & \text { if } S=J, \\
\frac{\alpha|J|+1-\alpha}{(1+\alpha)|J|+1-\alpha}, & S \cap J=\{j\}, \\
0, & \text { if } 2 \leq|S \cap J| \leq|J|-1,\end{cases}
\end{aligned}
$$

for all winning coalitions $S \subseteq N$ and all minor players $j \in S$.

Proof. It can easily be shown that $x$ is stable with respect to $P$. We show that $x$ is the unique stable power configuration. Let therefore $y \in \Delta(v)$ be stable with respect as well. By Lemma 3.2.12 we have $y_{i}(S)=1$ for all winning $S \subseteq N$ with $2 \leq|S \cap J| \leq|J|-1$. Consequently, $d_{i}(\{i, j\}, y)=1$ for all $j \in J$. Hence,

$$
y_{j}(\{i, j\})=\frac{\alpha+(1-\alpha) y_{j}(J)}{1+\alpha+(1-\alpha) y_{j}(J)}=1-\frac{1}{1+\alpha+(1-\alpha) y_{j}(J)} .
$$

We also have that

$$
y_{j}(J)=\frac{\alpha+(1-\alpha) y_{j}(\{i, j\})}{\alpha|J|+(1-\alpha) \sum_{k \in J} y_{k}(\{i, k\})} .
$$

Let $Y=\sum_{k \in J} y_{k}(\{i, k\})$. Then

$$
y_{j}(J)=\frac{1-\frac{1-\alpha}{1+\alpha+(1-\alpha) y_{j}(J)}}{|J|-(1-\alpha) Y}
$$

for all $j \in J$. Hence, $y_{j}$ does not depend on $j$, so we must have $y_{j}(J)=y_{k}(J)$ for all $j, k \in J$. By efficiency of $P, y_{j}(J)=\frac{1}{|J|}$. For the remaining coalitions $S$ it can now easily be shown that $x_{k}(S)=y_{k}(S)$ for all $k \in S$.

We see immediately that the only candidates for core stable coalitions are $J$ and $\{i, j\}$ for all $j \in J$. Hence, the following corollary can easily be derived.

Corollary 3.3.6. Let $a_{i J}$ be an apex game on $N$ and $x \in \Delta\left(a_{i J}\right)$ be stable with respect to $P$. Then there is a core stable coalition withr espect to $x$. In particular,

$$
\begin{aligned}
J \text { is core stable } \quad \text { if and only if } & |J| \leq \sqrt{\frac{1}{\alpha}}+1, \\
\{i, j\} \text { is core stable for all } j \in J \quad \text { if and only if } & |J| \geq \sqrt{\frac{1}{\alpha}}+1,
\end{aligned}
$$

and there are no other core stable coalitions. 
The existence of a core stable coalition for each apex game is a very nice feature of the power configuration $x$, particularly, as we have shown in Chapter 2 that there are no core stable coalitions for hedonic coalition formation games which are derived from an apex game together with the Shapley-Shubik index or the Banzhaf-Coleman index (Corollary 2.4.12).

Before we turn to the egalitarian solution we give the following remark on the relation between different stability notions.

Remark 3.3.7. From Corollary 3.3 .6 it becomes clear that core stability does not imply individual stability: The coalition $\{i, j\}$ can never be individually stable as $x_{i}(\{i\} \cup J \backslash\{j\})>x_{i}(\{i, j\})$ for all $j \in J$. Coalition $J$ is Nash stable if and only if $|J|<\sqrt{\frac{1}{\alpha}}+1$. However, the original definition of Nash stability (Bogomolnaia and Jackson, 2002) applies on a partition of $N$ and states that each coalition in this partition must be Nash stable in our sense. If we apply this definition on the partition $\{\{i\}, J\}$, we see that player $i$ would prefer to join $J$. Hence, there is a discrepancy between the original notion and our Definition 3.3.1. This is not the case when we talk about individual stability: If $S$ is a winning coalition and $x_{i}(S \cup\{i\})>0$, coalition $S$ would never allow player $i$ to join $S$ as at least one player $j \in S$ would lose power.

From Theorem 3.2.7 we know that there is a stable power configuration with respect to $E$ for each proper monotonic simple game. We will prove that it is unique if an apex game is under consideration. For this purpose we need the following upper bound for the power of a player $i$ in any coalition.

Lemma 3.3.8. Let $v$ be a proper monotonic simple game and $x \in \Delta(v)$ be stable with respect to $E$. Then $x_{i}(S) \leq 1$ for all $S \subseteq N$ and all $i \in S$.

Proof. Assume that there is $S \subseteq N$ and $i \in S$ such that $x_{i}(S)>1$ and let $\varepsilon=x_{i}(S)-1$. Since

$$
\begin{aligned}
1+\varepsilon & =x_{i}(S) \\
& \leq \alpha+(1-\alpha) d_{i}^{o}(S)+\frac{1}{|S|}\left(1-\alpha-(1-\alpha) \sum_{j \in S} d_{j}^{o}(S)\right) \\
& \leq \frac{|S|-1}{|S|}(1-\alpha) d_{i}^{o}(S)+\frac{1}{|S|}+\frac{|S|-1}{|S|} \alpha
\end{aligned}
$$

we find

$$
d_{i}^{o}\left(S_{1}\right) \geq \frac{1+\frac{|S|}{|S|-1} \varepsilon-\alpha}{1-\alpha} \geq 1+\frac{|S|}{|S|-1} \varepsilon
$$


Let $T_{1} \in \mathcal{T}_{i}^{S}(x)$, i.e. $x_{i}\left(T_{1}\right) \geq 1+\frac{|S|}{|S|-1} \varepsilon>x_{i}(S)$. Then we find for the same reasons as before $d_{i}^{o}\left(T_{1}\right)>x_{i}\left(T_{1}\right)$. Let now $T_{k+1} \in \mathcal{T}_{i}^{T_{k}}(x)$ for all $k \geq 1$. With the same arguments we have $x_{i}\left(T_{k+1}\right)>x_{i}\left(T_{k}\right)$ for all $k \geq 1$. But this is impossible since there is only a finite number of coalitions.

With this result at hand we can now calculate a stable power configuration and show that it is unique. The proof contains several linear equation systems which depend on the parameter $\alpha$. Instead of solving them step by step we present only the (unique) solutions and analyse their properties. ${ }^{3}$

Theorem 3.3.9. Let $a_{i J}$ be an apex game on $N=\{i\} \cup J$.

1. If $|J|=3$, the unique $x^{*} \in \Delta\left(a_{i J}\right)$ which is stable with respect to $E$ is given by

$$
\begin{aligned}
& x_{i}^{*}(S)= \begin{cases}\frac{1}{4}, & \text { if } S=N, \\
\frac{1}{2}+\frac{\alpha^{2}-1}{2 \alpha^{2}-4 \alpha-4}, & \text { if }|S \cap J|=1, \\
\frac{1}{3}+\frac{1+\alpha}{2+2 \alpha-\alpha^{2}}, & \text { if }|S \cap J|=2,\end{cases} \\
& x_{j}^{*}(S)= \begin{cases}\frac{1}{4}, & \text { if } S=N, \\
\frac{1}{3}, & \text { if } S=J, \\
\frac{1}{2}-\frac{\alpha^{2}-1}{2 \alpha^{2}-4 \alpha-4}, & \text { if } S \cap J=\{j\}, \\
\frac{2}{3}-\frac{1+\alpha}{2+2 \alpha-\alpha^{2}}, & \text { if }|S \cap J|=2\end{cases}
\end{aligned}
$$

for all winning coalitions $S \subseteq N$ and all minor players $j \in S$.

2. If $|J| \geq 4$, the unique $x^{*} \in \Delta\left(a_{i J}\right)$ which is stable with respect to $E$ is given by

$$
\begin{aligned}
& x_{i}^{*}(S)= \begin{array}{ll}
\frac{1}{|N|}, & \text { if } S=N, \\
1-\frac{\alpha}{2}-\frac{1-\alpha}{2|J|}, & \text { if }|S \cap J|=1, \\
1, & \text { if } 2 \leq|S \cap J| \leq|J|-2, \\
1-\frac{|J|-1}{|J|}\left(\frac{\alpha(1-\alpha)}{2}+\frac{(1-\alpha)^{2}}{2|J|}\right), & \text { if }|S \cap J|=|J|-1
\end{array} \\
& x_{j}^{*}(S)= \begin{cases}\frac{1}{|N|}, & \text { if } S=N, \\
\frac{1}{|J|}, & \text { if } S=J, \\
\frac{\alpha}{2}+\frac{1-\alpha}{2|J|}, & \text { if }|S \cap J|=1, \\
0, & \text { if } 2 \leq|S \cap J| \leq|J|-2, \\
\frac{|J|-1}{|J|}\left(\frac{\alpha(1-\alpha)}{2}-\frac{(1-\alpha)^{2}}{2|J|}\right), & \text { if }|S \cap J|=|J|-1\end{cases}
\end{aligned}
$$

for all winning coalitions $S \subseteq N$ and all minor players $j \in S$.

\footnotetext{
${ }^{3}$ The maple source codes of the respective solutions are available upon request.
} 


\section{Proof.}

1. It is easy to verify that $x^{*}$ is stable with respect to $E$, we show that $x^{*}$ is unique. Let therefore $x$ be stable with respect to $E$. Since $x_{i}(\{i, j\}) \leq 1$ for all $j \in J$, we have

$$
\begin{aligned}
x_{i}(\{i\} \cup J \backslash\{j\}) & =\frac{1}{3}+\frac{2}{3} \alpha+\frac{2}{3}(1-\alpha) x_{i}(\{i, j\}) \\
& \geq x_{i}(\{i, j\}) .
\end{aligned}
$$

We show that there is no $j \in J$ such that $x_{i}(\{i, j\})>x_{i}(\{i, j, k\})$ for all $k \in J \backslash\{j\}$. Assume that there is such $j \in J$. In this case we have

$$
x_{i}(\{i, j\})>x_{i}(\{i, j, k\}) \geq x_{i}(\{i, l\})
$$

for all $k, l \in J \backslash\{j\}, k \neq l$. Hence, we have $\{i, j\} \in \mathcal{T}_{i}^{\{i, k\}}$ for all $k \in J \backslash\{j\}$; thus $d_{i}^{o}(\{i, k\})=x_{i}(\{i, j\})$. Therefore, $x$ must solve the following equation system.

$$
\begin{aligned}
x_{i}(\{i, j\}) & =\frac{1}{2}+\frac{1-\alpha}{2} x_{i}(\{i, k, l\})-\frac{1-\alpha}{2} x_{j}(J) \\
x_{j}(\{i, j\}) & =\frac{1}{2}-\frac{1-\alpha}{2} x_{i}(\{i, k, l\})+\frac{1-\alpha}{2} x_{j}(J) \\
x_{i}(\{i, k\}) & =\frac{1}{2}+\frac{1-\alpha}{2} x_{i}(\{i, j\})-\frac{1-\alpha}{2} x_{k}(J) \\
x_{k}(\{i, k\}) & =\frac{1}{2}-\frac{1-\alpha}{2} x_{i}(\{i, j\})+\frac{1-\alpha}{2} x_{k}(J) \\
x_{i}(\{i, l\}) & =\frac{1}{2}+\frac{1-\alpha}{2} x_{i}(\{i, j\})-\frac{1-\alpha}{2} x_{l}(J) \\
x_{l}(\{i, l\}) & =\frac{1}{2}-\frac{1-\alpha}{2} x_{i}(\{i, j\})+\frac{1-\alpha}{2} x_{l}(J) \\
x_{i}(\{i, j, k\}) & =\frac{1}{3}+\frac{2 \alpha}{3}+\frac{2(1-\alpha)}{3} x_{i}(\{i, l\}) \\
x_{i}(\{i, j, l\}) & =\frac{1}{3}+\frac{2 \alpha}{3}+\frac{2(1-\alpha)}{3} x_{i}(\{i, k\}) \\
x_{i}(\{i, k, l\}) & =\frac{1}{3}+\frac{2 \alpha}{3}+\frac{2(1-\alpha)}{3} x_{i}(\{i, j\}) \\
x_{j}(J) & =\frac{1}{3}+\frac{2(1-\alpha)}{3} x_{j}(\{i, j\})-\frac{1-\alpha}{3} x_{k}(\{i, k\})-\frac{1-\alpha}{3} x_{l}(\{i, l\}) \\
x_{k}(J) & =\frac{1}{3}-\frac{1-\alpha}{3} x_{j}(\{i, j\})+\frac{2(1-\alpha)}{3} x_{k}(\{i, k\})-\frac{1-\alpha}{3} x_{l}(\{i, l\}) \\
x_{l}(J) & =\frac{1}{3}-\frac{1-\alpha}{3} x_{j}(\{i, j\})-\frac{1-\alpha}{3} x_{k}(\{i, k\})+\frac{2(1-\alpha)}{3} x_{l}(\{i, l\})
\end{aligned}
$$

The unique solution of this system delivers

$$
\begin{aligned}
x_{i}(\{i, j\}) & =\frac{2 \alpha^{4}-8 \alpha^{3}-9 \alpha^{2}+22 \alpha+11}{2 \alpha^{4}-14 \alpha^{3}+34 \alpha+14} \\
x_{i}(\{i, j, k\}) & =\frac{7 \alpha^{4}+2 \alpha^{3}-6 \alpha^{2}-40 \alpha-17}{3 \alpha^{4}-21 \alpha^{3}+51 \alpha+21} .
\end{aligned}
$$

It can now be shown that $x_{i}(\{i, j, k\})>x_{i}(\{i, j\})$ for all $\alpha \in[0,1]$ in contradiction to our assumption. Hence, there is no $j \in J$ such that $x_{i}(\{i, j\})>x_{i}(\{i, j, k\})$ for all $k \in J \backslash\{j\}$. 
Assume now that there is $j \in J$ such that $x_{i}(\{i, j, l\}) \geq x_{i}(\{i, j\})>$ $x_{i}(\{i, j, k\})$ for $j, l \in J \backslash\{j\}$. In this case we have $d_{i}^{o}(\{i, k\})=x_{i}(\{i, j, l\})$ and $d_{i}^{o}(\{i, l\})=x_{i}(\{i, j\})$. Hence, the only rows that change in the equation system compared to (3.4) are

$$
\begin{aligned}
x_{k}(\{i, k\}) & =\frac{1}{2}-\frac{1-\alpha}{2} x_{i}(\{i, j, l\})+\frac{1-\alpha}{2} x_{k}(J) \\
x_{i}(\{i, l\}) & =\frac{1}{2}+\frac{1-\alpha}{2} x_{i}(\{i, j\})-\frac{1-\alpha}{2} x_{l}(J) .
\end{aligned}
$$

In this case there is again for each $\alpha \in[0,1]$ a unique solution, in particular we have

$$
\begin{aligned}
x_{i}(\{i, j\}) & =\frac{4 \alpha^{4}-13 \alpha^{3}-6 \alpha^{2}+23 \alpha+10}{4 \alpha^{4}-19 \alpha^{3}+3 \alpha^{2}+35 \alpha+13} \\
x_{i}(\{i, j, k\}) & =\frac{8 \alpha^{4}+19 \alpha^{3}-21 \alpha^{2}-83 \alpha-31}{12 \alpha^{4}-57 \alpha^{3}+9 \alpha^{2}+105 \alpha+39} .
\end{aligned}
$$

It can now be shown that $x_{i}(\{i, j, k\})>x_{i}(\{i, j\})$ for all $\alpha \in[0,1]$. Again a contradiction to our assumption, that is there is no $j \in J$ such that $x_{i}(\{i, j, l\}) \geq x_{i}(\{i, j\})>x_{i}(\{i, j, k\})$ for $j, l \in J \backslash\{j\}$.

After we ruled out the previous two possibilities, it must now be the case that $d_{i}^{o}(\{i, j\})=x_{i}(\{i\} \cup J \backslash\{j\})$ for all $j \in J$. Hence, a stable power configuration must solve

$$
\begin{aligned}
x_{i}(\{i, j\}) & =\frac{1}{2}+\frac{1-\alpha}{2} x_{i}(\{i, k, l\})-\frac{1-\alpha}{2} x_{j}(J) \\
x_{j}(\{i, j\}) & =\frac{1}{2}-\frac{1-\alpha}{2} x_{i}(\{i, k, l\})+\frac{1-\alpha}{2} x_{j}(J) \\
x_{i}(\{i, k\}) & =\frac{1}{2}+\frac{1-\alpha}{2} x_{i}(\{i, j, l\})-\frac{1-\alpha}{2} x_{k}(J) \\
x_{k}(\{i, k\}) & =\frac{1}{2}-\frac{1-\alpha}{2} x_{i}(\{i, j, l\})+\frac{1-\alpha}{2} x_{k}(J) \\
x_{i}(\{i, l\}) & =\frac{1}{2}+\frac{1-\alpha}{2} x_{i}(\{i, j, k\})-\frac{1-\alpha}{2} x_{l}(J) \\
x_{l}(\{i, l\}) & =\frac{1}{2}-\frac{1-\alpha}{2} x_{i}(\{i, j, k\})+\frac{1-\alpha}{2} x_{l}(J) \\
x_{i}(\{i, j, k\}) & =\frac{1}{3}+\frac{2 \alpha}{3}+\frac{2(1-\alpha)}{3} x_{i}(\{i, l\}) \\
x_{i}(\{i, j, l\}) & =\frac{1}{3}+\frac{2 \alpha}{3}+\frac{2(1-\alpha)}{3} x_{i}(\{i, k\}) \\
x_{i}(\{i, k, l\}) & =\frac{1}{3}+\frac{2 \alpha}{3}+\frac{2(1-\alpha)}{3} x_{i}(\{i, j\}) \\
x_{j}(J) & =\frac{1}{3}+\frac{2(1-\alpha)}{3} x_{j}(\{i, j\})-\frac{1-\alpha}{3} x_{k}(\{i, k\})-\frac{1-\alpha}{3} x_{l}(\{i, l\}) \\
x_{k}(J) & =\frac{1}{3}-\frac{1-\alpha}{3} x_{j}(\{i, j\})+\frac{2(1-\alpha)}{3} x_{k}(\{i, k\})-\frac{1-\alpha}{3} x_{l}(\{i, l\}) \\
x_{l}(J) & =\frac{1}{3}-\frac{1-\alpha}{3} x_{j}(\{i, j\})-\frac{1-\alpha}{3} x_{k}(\{i, k\})+\frac{2(1-\alpha)}{3} x_{l}(\{i, l\}) .
\end{aligned}
$$

We find that in this case the unique solution is given in (3.3).

2. It is straightforward to verify that $x^{*}$ is stable with respect to $E$, we show that $x^{*}$ is unique. Let therefore $x$ be stable with respect to $E$ and define

$$
\mathcal{S}=\{S \subseteq N ; i \in S,|S \cap J|=2\}
$$


Let $S_{1} \in \mathcal{S}$ be such that $x_{i}\left(S_{1}\right) \geq x_{i}(S)$ for all $S \in \mathcal{S}$ and let $S_{2} \in \mathcal{S}$ such that $S_{1} \cap S_{2}=\{i\}$ and $x_{1}\left(S_{2}\right) \geq x_{1}(S)$ for all $S \in \mathcal{S}$ with $S \cap S_{1}=\{i\}$. Then $d_{j}\left(S_{k}\right)=0$ for $k=1,2$ and all $j \in S_{k} \cap J$. Hence,

$$
\begin{aligned}
x_{i}\left(S_{1}\right) & \geq \alpha+(1-\alpha) x_{i}\left(S_{2}\right)+\frac{1}{3}\left(1-\alpha-(1-\alpha) x_{i}\left(S_{2}\right)\right) \\
& =\frac{1+2 \alpha}{3}+\frac{2-2 \alpha}{3} x_{i}\left(S_{2}\right) .
\end{aligned}
$$

We also see for the same reasons that $x_{i}\left(S_{2}\right) \geq \frac{1+2 \alpha}{3}+\frac{2-2 \alpha}{3} x_{i}\left(S_{1}\right)$, so that

$$
\begin{aligned}
x_{i}\left(S_{1}\right) & \geq \frac{1+2 \alpha}{3}+\frac{2-2 \alpha}{3}\left(\frac{1+2 \alpha}{3}+\frac{2-2 \alpha}{3} x_{i}\left(S_{1}\right)\right) \\
& =\frac{5+8 \alpha-4 \alpha^{2}}{9}+\frac{4-8 \alpha+4 \alpha^{2}}{9} x_{i}\left(S_{1}\right) .
\end{aligned}
$$

Hence, $x_{i}\left(S_{1}\right) \geq 1$ and for the same reasons $x_{i}\left(S_{2}\right) \geq 1$. By Lemma 3.3.8 we have $x_{i}\left(S_{1}\right)=1$.

If $S$ is such that $2 \leq|S \cap J| \leq|J|-2$ then $d_{i}(S)=1, d_{j}(S)=0$ for all $j \in S \cap J$, and hence, $x_{i}(S)=1$ and $x_{j}(S)=0$. Similar to the proof of Theorem 3.3.5 we show that $x_{j}(J)=x_{k}(J)$ for all $j, k \in J$ and conclude $x_{j}(J)=\frac{1}{|J|}$. Hence, we have

$$
x_{i}(\{i, j\})=1+\frac{1}{2}\left(1-1-\alpha-(1-\alpha) \frac{1}{|J|}\right)=1-\frac{\alpha}{2}-\frac{1-\alpha}{2|J|}
$$

for all $j \in J$. Hence, $x_{j}(\{i, j\})=\frac{\alpha}{2}+\frac{1-\alpha}{2|J|}$. Finally,

$$
\begin{aligned}
x_{i}(\{i\} \cup J \backslash\{j\}) & =\frac{1}{|J|}+\frac{|J|-1}{|J|} \alpha+\frac{|J|-1}{|J|}(1-\alpha)\left(1-\frac{\alpha}{2}-\frac{1-\alpha}{2|J|}\right) \\
& =1-(1-\alpha) \frac{|J|-1}{|J|}\left(\frac{\alpha}{2}+\frac{1-\alpha}{2|J|}\right)
\end{aligned}
$$

and therefore $x_{k}(\{i\} \cup J \backslash\{j\})=(1-\alpha) \frac{|J|-1}{|J|}\left(\frac{\alpha}{2}+\frac{1-\alpha}{2|J|}\right)$ for all $k \in J \backslash$ $\{j\}$.

Note that in case of $\tilde{E}$ we have that $d_{k}(S) \leq 1$ for all winning coalitions $S$ and all $k \in S$. In particular, for each winning coalition $S$ except $J$ we find that $d_{k}(S) \geq-\frac{1}{|S|}\left(1-\sum_{l \in S} d_{l}(S)\right)$ for all $k \in S$. Hence, we have that for $x \in \Delta(v)$ which is stable with respect to $\tilde{E}$ it holds true that $x_{k}(S)=E_{i}(S, v(S), d(S))$. 
With similar arguments as in the proof of Theorem 3.3.9 it can be shown that $x_{j}(J)=\frac{1}{|J|}$ for all $j \in J$. Hence, stable payoff configurations with respect to $E$ and with respect to $\tilde{E}$ coincide on all apex games. We close this section with the following corollary on the existence of core stable coalitions.

Corollary 3.3.10. Let $a_{i J}$ be an apex game on $N=\{i\} \cup J$ and let $x \in \Delta\left(a_{i J}\right)$ be stable with respect to $E$.

1. Let $|J|=3$. Then $J$ is core stable if and only if $\alpha \leq \frac{\sqrt{3}-1}{2}$. In this case $J$ is the only core stable coalition. Further $\{i, j\}$ is core stable for each $j \in J$ if and only if $\alpha=1$. In this case there are no other core stable coalitions. If $\alpha \in\left(\frac{\sqrt{3}-1}{2}, 1\right)$ there are no core stable coalitions.

2. Let $|J| \geq 4$. Then $J$ is core stable if and only if $|J| \leq \frac{1+\alpha}{\alpha}$. In this case $J$ is the only core stable coalition. Further $\{i, j\}$ is core stable for each $j \in J$ if and only if $\alpha=1$. In this case there are no other core stable coalitions. If $\alpha \in\left(\frac{1}{|J|-1}, 1\right)$ then there are no core stable coalitions.

Proof. Let $x$ be the unique stable power configuration with respect to $a_{i J}$.

1. Let $|J|=3$. Then $x_{j}(\{i, j\}) \leq \frac{1}{3}=x_{j}(J)$ if and only if $\alpha \leq \frac{\sqrt{3}-1}{2}$. As $x_{j}(\{i, j, k\}) \leq x_{j}(\{i, j\})$, we have that $J$ is core stable if and only if $\alpha \leq \frac{\sqrt{3}-1}{2}$. In this case each coalition of type $\{i, j, k\}$ is blocked by $J$ and each coalition of type $\{i, j\}$ is blocked by $\{i\} \cup J \backslash\{j\}$. If $1>\alpha>\frac{\sqrt{3}-1}{2}$ then $J$ is blocked by $\{i, j\},\{i, j\}$ is blocked by $\{i\} \cup J \backslash\{j\}$, and $\{i\} \cup J \backslash\{j\}$ is blocked by $J$. If $\alpha=1$ then $J$ is blocked by $\{i, j\}$ and $\{i\} \cup J \backslash\{j\}$ is blocked by $J$. However, $\{i, j\}$ is not blocked by $\{i\} \cup J \backslash\{j\}$, since $x_{k}(\{i\} \cup J \backslash\{j\})=0$ for all $k \in J \backslash\{j\}$.

2. Let $|J|=4$. Then $x_{j}(\{i, j\}) \leq \frac{1}{|J|}=x_{j}(J)$ if and only if $\alpha \leq \frac{1}{|J|-1}$. We have that $x_{j}(\{i\} \cup J \backslash\{k\}) \leq x_{j}(\{i, j\})$ for all $k \in J \backslash\{j\}$, hence, $J$ is core stable if and only if $\alpha \leq \frac{1}{|J|-1}$ or equivalently $|J| \leq \frac{1+\alpha}{\alpha}$. In this case each winning coalition which contains $i$ and at least two minor players is blocked by $J$ and $\{i, j\}$ is blocked by $\{i\} \cup J \backslash\{j\}$. If $\alpha=1$ then $J$ is blocked by $\{i, j\}$ and $\{i\} \cup J \backslash\{j\}$ is blocked by $J$. However, $\{i, j\}$ is not blocked by $\{i\} \cup J \backslash\{j\}$, since $x_{k}(\{i\} \cup J \backslash\{j\})=0$ for all $k \in J \backslash\{j\}$. If $\frac{1}{|J|-1}<\alpha<1$ then $J$ is blocked by $\{i, j\},\{i, j\}$ is blocked by $\{i\} \cup J \backslash\{j\}$, and each coalition which contains $i$ and at least two minor players is blocked by $J$. 


\subsection{Infeasible Coalitions}

In many applications of simple games the formation of certain coalitions is impossible. This might be because of legal issues (such as antitrust legislation) or simply because some political parties have so different interests that they cannot work together. So far, we ignored such restrictions. However, as the disagreement points of players depend on their outside options, we should guarantee that a player cannot use his hypothetical power in a coalition which will never form.

We say that $\mathcal{R} \subseteq \mathcal{P}$ is a coalition restriction if $\{i\} \in \mathcal{R}$ for all $i \in N$. This condition simply says that each player can stay alone, in particular, each player has the outside option to stay alone.

Definition 3.4.1. Let $F$ be a bargaining solution, $\mathcal{R}$ be a coalition restriction, and $v$ be a simple game. A power configuration $x \in \Delta(v)$ is called stable with respect to $F$ under $\mathcal{R}$ if for all winning coalitions $S \in \mathcal{R}$ and all $i \in S$ the following holds.

$$
\begin{aligned}
x_{i}(S) & =F_{i}(S, v(S), d(S, x)) \\
d_{i}(S, x) & =\alpha d_{i}^{m}(S)+(1-\alpha) d_{i}^{o}(S, x) \\
d_{i}^{o}(S, x) & =\max _{T \in \mathcal{R}, T \subseteq N \backslash S} x_{i}(T \cup\{i\}) .
\end{aligned}
$$

It is easy to show that the proofs in Section 3.2 hold true for each coalition restriction $\mathcal{R}$. Theorem 3.3.3 remains true as well: Let $v$ be a proper monotonic simple game. If $\mathcal{R}$ contains at least one winning coalition and if $x$ is stable with respect to a fair and individually rational bargaining solution for $\alpha=0$ then there is a winning coalition $S \in \mathcal{R}$ which is both Nash and internally stable.

In this section we do not focus on the adaptation of the respective proofs but we will return to our initial Example 3.1.1. The political interests of the five parties in the German Bundestag make it impossible that FDP and Linke, or $\mathrm{CDU} / \mathrm{CSU}$ and Linke will ever cooperate. Therefore, let

$$
\mathcal{R}=\{S \subseteq N ; \text { if } 4 \in S \text { then } 1,2 \notin S\} .
$$

It can be shown that a stable power configuration with respect to $E$ under $\mathcal{R}$ must satisfy the equation system

$$
\begin{aligned}
x_{1}(\{1,2\}) & =\frac{1}{2}+\frac{1-\alpha}{2} x_{1}(\{1,3,5\}) \\
x_{1}(\{1,3\}) & =\frac{1}{2}+\frac{1-\alpha}{2} x_{1}(\{1,2,5\}) \\
x_{1}(\{1,3,5\}) & =\frac{1+\alpha}{3}+\frac{2}{3}(1-\alpha) x_{1}(\{1,2\}) \\
x_{1}(\{1,2,5\}) & =\frac{1+\alpha}{3}+\frac{2}{3}(1-\alpha) x_{1}(\{1,3\}) .
\end{aligned}
$$

The unique solution of this system is

$$
\begin{gathered}
x_{1}(\{1,2\})=x_{1}(\{1,3\})=\frac{2-\frac{1}{2} \alpha^{2}}{2+2 \alpha-\alpha^{2}} \\
x_{1}(\{1,3,5\})=x_{1}(\{1,2,5\})=\frac{2}{2+2 \alpha-\alpha^{2}} .
\end{gathered}
$$


We can further calculate

$$
x_{5}(\{1,3,5\})=x_{5}(\{1,2,5\})=\frac{1-2 \alpha}{3}-\frac{1}{3} x_{i}(\{1,3\})=-\frac{1}{6} \frac{4 \alpha+9 \alpha^{2}-4 \alpha^{2}}{2+2 \alpha-\alpha^{2}}<0 .
$$

Hence, $\{1,3,5\}$ and $\{1,2,5\}$ are neither internally stable nor individually rational. Finally we have

$$
x_{2}(\{1,2\})=x_{3}(\{1,3\})=1-\frac{2-\frac{1}{2} \alpha^{2}}{2+2 \alpha-\alpha^{2}}
$$

and see that the coalitions $\{1,2\}$ and $\{1,3\}$ are the only core stable coalitions. This is in line with reality as the current government consists of players 1 and 2 .

The power configuration $x$ is not individually rational since $x_{5}(\{1,3,5\})<0$. Hence, in this case $E$ and $\tilde{E}$ do not coincide. Let us now focus on $\tilde{E}$. Let $x$ be stable with respect to $\tilde{E}$ under $\mathcal{R}$. Then

$$
x_{1}(\{1, j\})=\frac{1}{2}+\frac{1-\alpha}{2} d_{1}(\{1, j\})
$$

for $j=2,3$. First, assume $d_{1}^{o}(\{1,2\}, x)=x_{1}(\{1,3\})$ and $d_{1}^{o}(\{1,3\}, x)=$ $x_{1}(\{1,2\})$. In this case $x$ must solve

$$
\begin{aligned}
& x_{1}(\{1,2\})=\frac{1}{2}+\frac{1-\alpha}{2} x_{1}(\{1,3\}) \\
& x_{1}(\{1,3\})=\frac{1}{2}+\frac{1-\alpha}{2} x_{1}(\{1,2\}) .
\end{aligned}
$$

We find that in this case $x_{1}(S)=\frac{1}{1+\alpha}$ for all winning coalitions $S \in \mathcal{R}$. Further $x_{j}(\{1, j\})=x_{j}(\{1,5, j\})=\frac{\alpha}{1+\alpha}$ for $j=2,3$ and $x_{5}(S)=0$ for all winning $S \in \mathcal{R}$. To verify that $x$ is actually stable, we have to show that the initial choice of outside options is consistent with $x$, i.e. that each player uses his best outside option. But this is clear since the only player with a positive outside option is player 1 and $x_{1}(S)=\frac{1}{1+\alpha}$ for all winning $S \in \mathcal{R}$.

We have mentioned before that we do not have evidence how $\alpha$ should be chosen. However, given the fact that the German cabinet consists of 16 ministers of which 11 are member of CDU/CSU, we can at least get an idea of $\alpha$. Under the assumption that the allocation of cabinets seats displays the power of the two parties, we find that $\alpha$ must solve

$$
x_{i}(\{1,2\})=\frac{1}{1+\alpha}=\frac{11}{16}
$$

for the constrained egalitarian solution. This delivers $\alpha \approx 0.455$. For the egalitarian solution we can use the same argument to find $\alpha \approx 0.487$. 


\subsection{Conclusion}

Coalition formation in simple games contains two parts: The forming of coalitions and the distribution of power within coalitions. We built a model in which these two parts are interdependent, the distribution of power depends on the formation of coalitions and the formation of coalitions depends on the distribution of power. We interpreted the distribution problem as a bargaining (or bankruptcy) problem and showed that under very weak conditions on the bargaining solutions we can find a power configuration which is stable with respect to renegotiations.

We pointed out that essentially two issues are crucial for the power of a player within a coalition. First, his marginal contribution, as a pivotal player will always be more powerful than a player who is not necessary for the surviving of a coalition. Second, and this is the new approach, his outside option. We can also interpret the outside option as opportunity costs: A player who has a chance to be in a very powerful position in a different winning coalition must somehow be convinced not to leave. In the paper we showed several results for specific convex combinations between these two values. However, we do not have any empirical evidence yet, how they should be weighted.

Besides the very natural motivation of this stable power configuration, we showed that it has further useful properties: First of all, it allows to take into account that there might be coalitions which will never form for any external reasons. Second, under some additional conditions it guarantees the existence of a coalition which is both internally and individually stable for each proper monotonic simple game.

We can think of several challenges which can now be targeted: Empirical evidence for the applicability of the model is the first. In particular, it will be interesting to investigate the implicit values of $\alpha$. Second, the model might be extended to general transferable utility games. In this case we would interpret the outside option of a player in a coalition as opportunity costs. Particularly, these costs will depend on the partition rather than on a coalition. A transformation of the game in a hedonic version will therefore lead to a hedonic game with externalities (Bloch and Dutta, 2011). 


\section{Chapter 4}

\section{Mutual Control Structures}

\subsection{Introduction}

A mutual control structure refers to a situation in which agents exercise control on each other. Typical examples occur in the area of corporate governance: there is a conglomerate of firms and investment companies who control each other, specifically by possessing shares or stocks. In such situations the question arises who are, ultimately, in control, and how much power the different agents have. See Crama and Leruth (2011) for a recent overview of both theoretical and empirical literature in this area. The purpose of the present paper is to answer these questions and add to the literature by developing a general game-theoretic model.

Formally, a mutual control structure $C$ will be a map assigning to each nonempty coalition - i.e., a subset of a given finite set of players $N$ - another coalition. The interpretation of $C(S)=T$ is, that each player of $T$ is controlled by the coalition $S$. For instance, $i \in T$ is a firm, and the coalition $S$ of firms or investment companies has a majority of the shares of firm $i$. We impose the natural condition of monotonicity: if $S$ controls $T$, then any coalition containing $S$ also controls $T$. While the mutual control structure $C$ thus captures direct control, it does not necessarily capture indirect control. The latter means that whenever $S$ controls $T$ and $S$ and $T$ jointly control $R$, then $S$ indirectly controls $R$. Thus, formally, one would like to have that, if $T \subseteq C(S)$ and $R \subseteq C(S \cup T)$, then also $R \subseteq C(S)$. In our example, if $j$ is a firm in $R$ and $S$ and $T$ jointly have a majority of the shares of $j$, then $S$ controls $j$ since $S$ controls all firms in $T$. A mutual control structure will be called invariant if it satisfies this condition. In the paper we start out by studying an algorithm which assigns to each mutual control structure its unique minimal invariant extension. 
Alternatively, a mutual control structure can be described by a vector of simple games. For each player, there is a simple game with as winning coalitions exactly those coalitions which control that player. There is a one-to-one correspondence between mutual control structures and vectors of monotonic simple games. We propose an updating procedure, in which players can be replaced by coalitions which are winning in the simple game describing who controls that player. We show that this updating procedure results in a unique minimal invariant extension of the vector of simple games, and that the associated mutual control structure is indeed the minimal invariant extension of the originally given mutual control structure.

The approach in the literature which seems to be closest related to ours, is Gambarelli and Owen (1994). This approach explicitly distinguishes between firms and investors. In what they call a reduction, all power is reduced to power of the investors, i.e., the firms leave the scene. The proposed reduction operation bears some resemblance to our algorithm of making a mutual control structure invariant. Gambarelli and Owen (1994)) end up with so-called consistent reductions which, however, are not necessarily unique, in contrast to our minimal invariant extensions. There are some other approaches in the literature aiming at establishing indirect control relations: see for instance Crama and Leruth (2007) and the already mentioned survey Crama and Leruth (2011).

In the second part of the chapter we consider invariant mutual control structures and develop a class of power indices, intended to capture the 'true' power of individual players. We impose four axioms which have a natural interpretation in the present framework. First, we set the power of null-players equal to zero a null-player is a player who neither contributes to controlling any other player, nor is controlled by any other player or coalition himself. Second, we impose that the sum of all assigned powers is the same over all invariant mutual control structures. This axiom replaces the usual 'efficiency' condition; it will easily follow that this axiom together with the null-player axiom actually makes this sum equal to zero. Third, we impose anonymity: the names of the players should not matter. Fourth, we impose a so-called transfer property, which says the following. For every player, the change in power when extending a mutual control structure $C^{\prime}$ to $C$ should be equal to the change in power when extending a mutual control structure $D^{\prime}$ to $D$, whenever exactly the same control relations are added going from $C^{\prime}$ to $C$ as when going from $D^{\prime}$ to $D$. This condition is called transfer property because it is related to the transfer property used by Dubey (1975) to characterize the Shapley value or Shapley-Shubik index (Shapley, 1953; Shapley and Shubik, 1954) for monotonic simple games. See also Dubey et al. (2005).

We characterize the class of power indices satisfying these four conditions. Each power index in this class corresponds to a weight vector of dimension $2 n-2$ (where $n$ is the number of players) and assigns to a player $i$ a weighted sum of 
dividends obtained in the simple games capturing the control undergone by the other players, diminished by dividends gathered by the other players in the game describing the control undergone by player $i$. The number $2 n-2=2(n-1)$ is twice the number of possible cardinalities of a (nontrivial) coalition: for each coalition there is a weight associated with control exercised by that coalition, and a weight associated with control undergone by that coalition. By adding a natural monotonicity condition we restrict this class to power indices associated with nonnegative weight vectors. By imposing more scaling we obtain a unique power index with all weights equal to 1 . This means that each player $i$ obtains the sum of all his Shapley-Shubik values in the games in which he contributes to controlling the other players, minus the sum of all Shapley-Shubik values of the other players in the game describing the control undergone by $i$.

The approach in this paper can be applied whenever elementary, direct control relations can be retrieved from the data - for instance by considering simple majority share holdings within a corporate structure and/or taking into account special voting rights (as in the Porsche-Volkswagen case in the next section). Next, indirect control relations can be determined, and to the resulting invariant mutual control structure a power index can be applied. It should be noted, however, that it is probably impossible to capture all potential situations within an area of application in a completely satisfying way. See the final section for some discussion.

In Section 4.2 we introduce and study mutual control structures, in Section 4.3 we consider the approach by simple games, and in Section 4.4 we develop our class of power indices. Section 4.5 concludes.

\subsection{Mutual Control Structures}

As before let $N=\{1, \ldots, n\}$ with $n \geq 2$ denote the set of players.

Definition 4.2.1. A mutual control structure (mcs) is a map $C: \mathcal{P}(N) \rightarrow \mathcal{P}(N)$ satisfying

(i) $C(\emptyset)=\emptyset$,

(ii) monotonicity: $C(S) \subseteq C(T)$ for all $S, T \in \mathcal{P}(N)$ with $S \subseteq T$.

The set of all mutual control structures is denoted by $\Gamma$.

If $i \in C(S)$ for some $i \in N$ and $S \in \mathcal{P}(N)$ then we say that player $i$ is controlled by coalition $S$. (For instance, the firms in $S$ hold a majority of the shares of firm $i$.) Similarly, we say that $S$ controls $C(S)$. Thus, the empty coalition controls no one, and a player controlled by a coalition $S$ is also controlled by any coalition $T$ containing $S$. 
Example 4.2.2. In 2008 the Porsche group started buying common stocks of Volkswagen AG on a large scale. On January 5, 2009, Porsche announced to own more than $50 \%$ of the common stocks. However, the original plan to take over Volkswagen failed and the founding of an integrated corporation was announced. Figure 1 is a simplified organization chart of this corporation. ${ }^{1}$ Although Porsche SE has the majority of voting rights of Volkswagen AG, Lower Saxony has a veto power according to two laws. ${ }^{2}$ These laws specify in particular that important decisions can be made only with $80 \%$ of the voting rights (of which Lower Saxony owns slightly more than $20 \%) .^{3}$ In the following, we assume that the group of 'others' does not vote, i.e., we ignore them in the description of the game. This is reasonable since these shareholders have only small amounts of shares and are never needed for control. ${ }^{4}$ We can describe the situation by a mutal control structure $C$. The players are Porsche Families (1), Qatar (2), Lower Saxony (3), Porsche SE (4), Volkswagen AG (5), and Porsche AG (6). For any coalition $S \subseteq N=\{1, \ldots, 6\}$ we have:

$$
4 \in C(S) \Leftrightarrow 1 \in S, 5 \in C(S) \Leftrightarrow\{2,3,4\} \subseteq S \text {, and } 6 \in C(S) \Leftrightarrow 5 \in S .
$$

Clearly, $C$ satisfies the conditions of Definition 4.2.1.

A mutual control structure does not necessarily capture 'indirect' control: if $S$ controls $T$, and $S$ and $T$ together control $R$, then for an arbitrary mutual control structure it is not necessarily the case that $S$ controls $R$. This property of 'indirect control' is captured by the following definition.

Definition 4.2.3. The mutual control structure $C$ is invariant if it satisfies indirect control: For all $S, T, R \in \mathcal{P}(N)$ with $T \subseteq C(S)$ and $R \subseteq C(S \cup T)$ we have $R \subseteq C(S)$. The set of all invariant mutual control structures is denoted by $\Gamma^{*}$.

Clearly, the control structure $C$ in Example 4.2.2 violates indirect control with $S=\{1,2,3\}, T=\{4\}$, and $R=\{5\}$.

\footnotetext{
${ }^{1}$ Porsche Families is an aggregation of several companies and holdings which are held by members of the families Porsche and Piëch. Qatar is an aggregation of several holdings which are held by the Qatar Investment Authority, Doha. Lower Saxony includes Hannoversche Beteiligungsgesellschaft mbH which is held by Lower Saxony. Volkswagen AG holds $100 \%$ of Porsche Holding Stuttgart GmbH which in turn holds $100 \%$ of Porsche AG. Hence, we ignore this holding. Others means investors which hold less than $3 \%$ of the shares and are therefore not mentioned in any reports.

${ }^{2}$ Namely, $§ 111$ AktG (Aktiengesetz) and $\S 4$ VWGmbHÜG (Gesetz über die Überführung der Anteilsrechte der Volkswagen Gesellschaft mit beschränkter Haftung in private Hand).

${ }^{3}$ Figure 1 describes the situation as it was before mid June, 2013. Meanwhile, Qatar has sold its shares in Porsche SE back to Porsche Families. For the sake of the example, however, we stick to the situation before mid June, 2013.

${ }^{4}$ Alternatively, we could include them as a player: this player would then be a null-player as defined in Section 4.4 .
} 


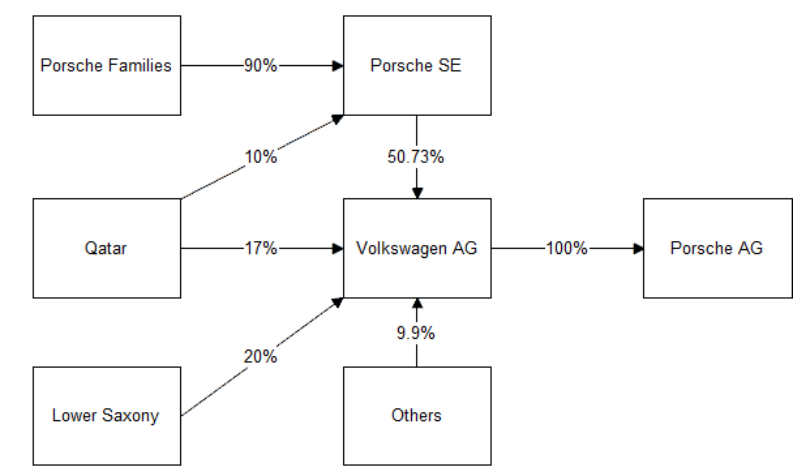

Figure 1: Porsche and VW voting rights by the end of 2012, based on the annual reports 2012 of Volkswagen AG and Porsche Automobil Holding SE GmbH.

The term 'invariant' reflects the fact that such a mutual control structure does not change if we add further control relations in the sense of the indirect control property: if $T$ is controlled by $S$ and $R$ is controlled by $S$ and $T$ jointly, then $R$ is already controlled directly by $S$ and, thus, adding this control relation does not change the mcs.

Note that an invariant mutual control structure $C$ is transitive: for all $S, T, R \in$ $\mathcal{P}(N)$ with $T \subseteq C(S)$ and $R \subseteq C(T)$ we have $R \subseteq C(S)$. This follows since $R \subseteq C(T)$ implies $R \subseteq C(S \cup T)$ by monotonicity of $C$.

Examples of (invariant) mutual control structures can be derived from monotonic simple games.

Example 4.2.4. Let $v \in \Sigma$ be a monotonic simple game and define $C: \mathcal{P}(N) \rightarrow$ $\mathcal{P}(N)$ by

$$
C(S)= \begin{cases}N & \text { if } v(S)=1 \\ \emptyset & \text { if } v(S)=0\end{cases}
$$

for all $S \in \mathcal{P}(N)$. Then $C(\emptyset)=\emptyset$, and $C(S) \subseteq C(T)$ for all $S, T \in \mathcal{P}(N)$ with $S \subseteq T$, so $C \in \Gamma$. Also, if $S, T, R \in \mathcal{P}(N)$ with $T \subseteq C(S)$ and $R \subseteq C(S \cup T)$ then either $T=\emptyset$ in which case $R \subseteq C(S)$ or $T \neq \emptyset$ in which case $C(S)=N$ and thus $R \subseteq C(S)$ as well. Hence, $C$ satisfies indirect control, so that $C \in \Gamma^{*}$.

The remainder of this section is devoted to the question how an arbitrary mutual control structure (mcs) can be turned into an invariant mutual control structure (imcs). This requires, in particular, that indirect control relations are incorporated explicitly into the mcs. Let $C$ be an arbitrary mcs. We define 
$C^{1}, C^{2}, \ldots \in \Gamma$ recursively by

$$
C^{k}(S)= \begin{cases}C(S), & \text { if } k=1, \\ C\left(C^{k-1}(S) \cup S\right) & \text { if } k>1,\end{cases}
$$

for each $S \in \mathcal{P}(N)$. Observe that in each step of this algorithm the new coalition controlled by $S$ is the coalition controlled by $S$ jointly with the coalition already controlled by $S$ according to the previous step. Thus, this definition is very much in the spirit of the definition of indirect control. Clearly, by monotonicity of $C$, there must be a natural number $p \geq 1$ such that for each $S \in \mathcal{P}(N)$ we have

$$
C(S)=C^{1}(S) \subseteq C^{2}(S) \subseteq \ldots \subseteq C^{p}(S)=C^{p+1}(S)=C^{p+2}(S)=\ldots
$$

Denote $C^{p}$ by $C^{*}$. Then we have the following result, which says that in $C^{*}$ adding players controlled by a coalition $S$ to $S$ does not enlarge the set of players controlled by $S$.

Lemma 4.2.5. Let $C \in \Gamma$. Then for each $S \in \mathcal{P}(N)$ we have $C^{*}\left(S \cup C^{*}(S)\right)=$ $C^{*}(S)$.

Proof. Let $p \geq 1$ be as in the definition of $C^{*}$ (i.e., as in (4.1)) and let $S \in \mathcal{P}(N)$. Then we have

$$
C\left(S \cup C^{*}(S)\right)=C\left(\left(S \cup C^{p}(S)\right)=C^{p+1}(S)=C^{*}(S),\right.
$$

hence,

$$
C^{2}\left(S \cup C^{*}(S)\right)=C\left(S \cup C^{*}(S) \cup C\left(S \cup C^{*}(S)\right)\right)=C\left(S \cup C^{*}(S)\right)=C^{*}(S) .
$$

By repeating this argument we find

$$
C^{*}\left(S \cup C^{*}(S)\right)=C^{p}\left(S \cup C^{*}(S)\right)=C^{*}(S) .
$$

A few further observations are collected in the following lemma. We omit the straightforward induction proofs. For $C, D \in \Gamma$, we write $C \subseteq D$ if $C(S) \subseteq D(S)$ for all $S \in \mathcal{P}(N)$.

Lemma 4.2.6. Let $C \in \Gamma$. Then $C^{k} \in \Gamma$ for each $k \geq 1$. If $D \in \Gamma$ with $C \subseteq D$, then $C^{k} \subseteq D^{k}$ for each $k \geq 1$.

We now show that $C^{*}$ is an invariant mes.

Proposition 4.2.7. Let $C \in \Gamma$. Then $C^{*} \in \Gamma^{*}$. 
Proof. In view of Lemma 4.2.6 we only still have to show indirect control of $C^{*}$. Let $S \in \mathcal{P}(N)$ and $T \subseteq C^{*}(S)$. Then by monotonicity of $C^{*}$ and Lemma 4.2.5 we have

$$
C^{*}(S) \subseteq C^{*}(S \cup T) \subseteq C^{*}\left(S \cup C^{*}(S)\right)=C^{*}(S),
$$

so that $C^{*}(S \cup T)=C^{*}(S)$. Hence, if $R \subseteq C^{*}(S \cup T)$ then $R \subseteq C^{*}(S)$, so that indirect control holds.

We call $D \in \Gamma^{*}$ an invariant extension of $C \in \Gamma$ if $C \subseteq D$. Clearly, $C^{*}$ is an invariant extension of $C$, but also $D \in \Gamma^{*}$ defined by $D(S)=N$ for all $S \in P(N)$ is an invariant extension of any mutual control structure. Call an invariant extension $D$ of $C$ minimal if $D(S) \subseteq D^{\prime}(S)$ for every $S \in P(N)$ and every invariant extension $D^{\prime}$ of $C$. Obviously, minimal invariant extensions are unique. We have the following result.

Proposition 4.2.8. Let $C \in \mathcal{C}$. Then $C^{*}$ is the (unique) minimal invariant extension of $C$.

Proof. Clearly, $C^{*}$ is an invariant extension of $C$. Let $D \in \Gamma^{*}$ be an invariant extension of $C$ and let $S \in P(N)$. It is sufficient to prove that $C^{r}(S) \subseteq D(S)$ for all $r \geq 1$. For $r=1$ this follows by definition of an invariant extension. Suppose the claim is true for $r-1$. Then $C^{r}(S)=C\left(S \cup C^{r-1}(S)\right) \subseteq D\left(S \cup C^{r-1}(S)\right)$ again by definition of an invariant extension. By the induction hypothesis, $C^{r-1}(S) \subseteq$ $D(S)$. Hence by indirect control of $D$ we obtain $C^{r}(S) \subseteq D(S)$.

The following example is the Porsche-Volkswagen case.

Example 4.2.9. For $C$ as in Example 4.2 .2 we have for all $S \in P(N)$ :

$$
\begin{aligned}
& 4 \in C^{*}(S) \Leftrightarrow 1 \in S \\
& 5 \in C^{*}(S) \Leftrightarrow\{2,3,4\} \subseteq S \text { or }\{1,2,3\} \subseteq S \\
& 6 \in C^{*}(S) \Leftrightarrow 5 \in S \text { or }\{2,3,4\} \subseteq S \text { or }\{1,2,3\} \subseteq S .
\end{aligned}
$$

In particular, in $\mathcal{C}^{*}$, Volkswagen AG (5) is controlled by the coalition of Porsche Families, Qatar, and Lower Saxony (1, 2, and 3, respectively).

The next example shows that different mutual control structures may result in the same invariant mcs.

Example 4.2.10. Let $N=\{1,2,3\}$ and let $C, D \in \mathcal{C}$ be defined by monotonicity and $C(\{1\})=\{2\}, C(\{2\})=\{3\}, C(\{3\})=\{1\}, D(\{1\})=\{3\}, D(\{2\})=\{1\}$, $D(\{3\})=\{2\}$. Then $C^{*}(S)=D^{*}(S)=N$ for each nonempty coalition $S$.

We conclude this section with a remark on the unions and intersection of invariant mutual control structures. This will be relevant in particular when we discuss axioms for power indices later on. 
Remark 4.2.11. We define $\cup$ and $\cap$ on $\mathcal{C}$ by

$$
\begin{aligned}
& (C \cup D)(S)=C(S) \cup D(S), \\
& (C \cap D)(S)=C(S) \cap D(S),
\end{aligned}
$$

for all $S \in \mathcal{P}(N)$. Clearly, $C \cup D, C \cap D \in \Gamma$ for all $C, D \in \Gamma$. Let $C, D \in \Gamma^{*}$ and consider $C \cap D$. If $T \subseteq(C \cap D)(S)$ and $R \subseteq(C \cap D)(S \cup T)$, then $R \subseteq C(S)$ and $R \subseteq D(S)$ by indirect control. Hence, $R \subseteq(C \cap D)(S)$. Thus, $C \cap D \in \Gamma^{*}$.

However, $C \cup D$ does not have to be invariant. Consider the following mutual control structures

$$
\begin{aligned}
& C(S)= \begin{cases}\{2\}, & \text { if } 1 \in S, \\
\emptyset, & \text { otherwise },\end{cases} \\
& D(S)= \begin{cases}\{3\} & \text { if } 2 \in S, \\
\emptyset & \text { otherwise. }\end{cases}
\end{aligned}
$$

Then $(C \cup D)(\{1\})=\{2\},(C \cup D)(\{1,2\})=\{2,3\}$, but $\{2,3\} \nsubseteq(C \cup D)(\{1\})$. So $C \cup D$ is not invariant.

In the next section we consider the representation of a mutual control structure as a vector of simple games.

\subsection{Control Structures and Simple Games}

Let $C$ be an mcs. Instead of writing for each coalition all players it controls, we may equivalently write for each player all coalitions by which it is controlled. Formally, for each $i \in N$ we define a simple game $w_{i}^{C}$ by

$$
w_{i}^{C}(S)= \begin{cases}1, & \text { if } i \in C(S), \\ 0, & \text { if } i \notin C(S) .\end{cases}
$$

This way with every $C \in \Gamma$ a vector of monotonic simple games $w^{C} \in \Sigma^{N}$ is associated. Conversely, for a vector of simple games $w \in \Sigma^{N}$ we can define an mcs $C^{w}$ by $i \in C^{w}(S): \Leftrightarrow w_{i}(S)=1$ for all $i \in N$ and $S \in \mathcal{P}(N)$. Clearly, these definitions determine a bijection between $\Gamma$ and $\Sigma^{N}$, and all statements and results for $\Gamma$ can be equivalently formulated for $\Sigma^{N}$ and conversely.

For an arbitrary $w \in \Sigma^{N}$ it is not necessarily the case that $C^{w}$ is invariant, i.e., $C^{w} \in \Gamma^{*}$. For each pair $i, j \in N$ with $i \neq j$ we now define a map $t_{i, j}: \Sigma^{N} \rightarrow \Sigma^{N}$, such that repeated application of these maps will transform a $w \in \Sigma^{N}$ into a $w^{*} \in \Sigma^{N}$ with the property that $C^{w^{*}}$ is invariant. Formally,

$$
t_{i, j}\left(w_{k}\right)(S)= \begin{cases}w_{k}(S), & \text { if } k \neq i \\ \widetilde{w}_{i}(S), & \text { if } k=i\end{cases}
$$


for each $k \in N$, where

$\widetilde{w}_{i}(S)= \begin{cases}1, & \text { if } w_{i}(S)=1, \\ 1, & \text { if } S=\left(S_{1} \backslash\{j\}\right) \cup S_{2} \text { for some } S_{1}, S_{2} \text { with } w_{i}\left(S_{1}\right)=w_{j}\left(S_{2}\right)=1, \\ 0, & \text { otherwise }\end{cases}$

for all $S \in \mathcal{P}(N)$. We call $t_{i, j}$ an elementary substitution. In $t_{i, j}(w)$, the set of winning coalitions in game $w_{i}$ is extended by replacing player $j$ in every winning coalition of $w_{i}$ by any winning coalition of $w_{j}$; i.e., $j$ is replaced by a coalition that controls $j$. This is, clearly, in the spirit of indirect control.

Example 4.3.1. Recall the mutual control structure $C$ from Example 4.2.2 (Porsche-Volkswagen), where $N=\{1, \ldots, 6\}$ and the minimal winning coalitions in $w^{C}$ are given in the following table. (We leave out the zero games.)

\begin{tabular}{ccc}
\hline$w_{4}^{C}$ & $w_{5}^{C}$ & $w_{6}^{C}$ \\
\hline$\{1\}$ & $\{2,3,4\}$ & $\{5\}$ \\
\hline
\end{tabular}

Applying the transformation $t_{5,4}$ yields:

\begin{tabular}{lcc}
\hline$w_{4}^{C}$ & $w_{5}^{C}$ & $w_{6}^{C}$ \\
\hline$\{1\}$ & $\{2,3,4\},\{2,3,1\}$ & $\{5\}$ \\
\hline
\end{tabular}

and, next, application of $t_{6,5}$ yields:

\begin{tabular}{lcc}
\hline$w_{4}^{C}$ & $w_{5}^{C}$ & $w_{6}^{C}$ \\
\hline$\{1\}$ & $\{2,3,4\},\{2,3,1\}$ & $\{5\},\{2,3,4\},\{2,3,1\}$ \\
\hline
\end{tabular}

The last table describes a simple game structure $w^{*}$ (with $w_{1}^{*}, w_{2}^{*}, w_{3}^{*}$ zero games) which will not further change under elementary substitutions, and we have that $C^{w^{*}}$ is equal to $C^{*}$, with $C^{*}$ as given in Example 4.2.9.

Elementary substitutions are closely related to the procedure constructing invariant mcs discussed in the preceding section. Clearly, since applying elementary substitutions on a vector of simple games $w$ can only increase the collections of winning coalitions, after finitely many steps we must obtain some $w^{*} \in \Sigma^{N}$ which is invariant under such substitutions. We show that the associated mcs $C^{w^{*}}$ is invariant and equal to the imcs $\left(C^{w}\right)^{*}$ obtained by applying the algorithm of the preceding section to $C^{w}$. We start with the following lemma.

Lemma 4.3.2. Let $w \in \Sigma^{N}$ and $i, j \in N$ with $i \neq j$. Then we have for all $r \geq 1$

1. $\left(C^{w}\right)^{r} \subseteq\left(C^{t_{i, j}(w)}\right)^{r}$.

2. $\left(C^{t_{i, j}(w)}\right)^{r} \subseteq\left(C^{w}\right)^{2 r}$. 
Proof. Write $u=t_{i, j}(w)$. For (1.), since $u(S) \geq w(S)$ for all $S \in \mathcal{P}(N)$, we have $C^{w}(S) \subseteq C^{u}(S)$ for all $S$, and the proof is complete by Lemma 4.2.6.

In order to prove (2.), let $S \in \mathcal{P}(N)$. The proof is by induction. First suppose $r=1$. Let $k \in C^{u}(S)$, i.e., $u_{k}(S)=1$. If $k \neq i$ then $w_{k}(S)=u_{k}(S)=1$, hence $k \in C^{w}(S)=\left(C^{w}\right)^{1}(S) \subseteq\left(C^{w}\right)^{2}(S)$. This argument also holds if $k=i$ and $w_{k}(S)=1$. Now suppose $k=i$ and $w_{k}(S)=0$. Then there are $S_{1}, S_{2} \in \mathcal{P}(N)$ with $w_{k}\left(S_{1}\right)=1, w_{j}\left(S_{2}\right)=1$, and such that $S=\left(S_{1} \backslash\{j\}\right) \cup S_{2}$. Hence $w_{j}(S) \geq w_{j}\left(S_{2}\right)=1$ so that $j \in C^{w}(S)=\left(C^{w}\right)^{1}(S)$ and $w_{k}\left(S \cup\left(C^{w}\right)^{1}(S)\right) \geq$ $w_{k}(S \cup\{j\}) \geq w_{k}\left(S_{1}\right)=1$. Hence $k \in\left(C^{w}\right)^{2}(S)$ also in this case.

Now let $r \geq 2$ and suppose (2.) holds for all $k \leq r-1$. Then

$$
\begin{aligned}
\left(C^{u}\right)^{r}(S) & =C^{u}\left(S \cup\left(C^{u}\right)^{r-1}(S)\right) \\
& \subseteq\left(C^{w}\right)^{2}\left(S \cup\left(C^{w}\right)^{2 r-2}(S)\right) \\
& =C^{w}\left(S \cup\left(C^{w}\right)^{2 r-2}(S) \cup C^{w}\left(S \cup\left(C^{w}\right)^{2 r-2}(S)\right)\right) \\
& =C^{w}\left(S \cup\left(C^{w}\right)^{2 r-2}(S) \cup\left(C^{w}\right)^{2 r-1}(S)\right) \\
& =C^{w}\left(S \cup\left(C^{w}\right)^{2 r-1}(S)\right) \\
& =\left(C^{w}\right)^{2 r}(S)
\end{aligned}
$$

by using the induction hypothesis and Lemma 4.2.6.

A consequence of this lemma is that, if we first apply an elementary substitution to a vector of simple games, the associated invariant mes does not change.

Corollary 4.3.3. Let $w \in \Sigma^{N}$ and let $i, j \in N$ with $i \neq j$. Then $\left(C^{w}\right)^{*}=$ $\left(C^{t_{i, j}(w)}\right)^{*}$.

Proof. Write $u=t_{i, j}(w)$ and take $p \in \mathbb{N}$ such that $\left(C^{w}\right)^{r}=\left(C^{w}\right)^{*}$ and $\left(C^{u}\right)^{r}=$ $\left(C^{u}\right)^{*}$ for all $r \geq p$. Let $S \in \mathcal{P}(N)$. Then

$$
\left(C^{w}\right)^{*}(S)=\left(C^{w}\right)^{p}(S) \subseteq\left(C^{u}\right)^{p}(S) \subseteq\left(C^{w}\right)^{2 p}(S)=\left(C^{w}\right)^{*}(S),
$$

where the two inclusions follow from Lemma 4.3.2. Hence, $\left(C^{u}\right)^{*}(S)=\left(C^{u}\right)^{p}(S)=$ $\left(C^{w}\right)^{*}(S)$.

As already observed, repeatedly applying elementary substitutions to a $w \in$ $\Sigma^{N}$ must result in some $w^{*} \in \Sigma^{N}$ which is invariant under further elementary substitutions, i.e., $t_{i, j}\left(w^{*}\right)=w^{*}$ for all $i, j \in N$ with $i \neq j$. This is so because by an elementary substitution the set of winning coalitions in each coordinate game can only expand. Let $T(w) \subseteq \Sigma^{N}$ denote the set of all such $w^{*}$, i.e., obtainable from $w$ by elementary substitutions and invariant under further elementary substitutions. Below (Corollary 4.3.6) we will actually show that $T(w)$ contains a unique element for every $w \in \Sigma^{N}$. 
In general, call $\bar{w} \in \Sigma^{N}$ an extension of $w \in \Sigma^{N}$ if $\bar{w}_{i}(S) \geq w_{i}(S)$ for every $i \in N$ and $S \in \mathcal{P}(N)$. Call $w \in \Sigma^{N}$ invariant if $t_{i, j}(w)=\bar{w}$ for all $i, j \in N$ with $i \neq j$. Call $\bar{w} \in \Sigma^{N}$ a minimal invariant extension of $w \in \Sigma^{N}$ if $\bar{w}$ is an invariant extension of $w$ and for every invariant extension $w^{\prime} \in \Sigma^{N}$ of $w$ we have $w_{i}^{\prime}(S) \geq \bar{w}_{i}(S)$ for all $i \in N$ and $S \in \mathcal{P}(N)$. Observe that, if a minimal invariant extension of $w$ exists, then it is unique.

We first show that, if $\bar{w}$ is an invariant extension of $w$, then it is an invariant extension of $w^{*}$ for every $w^{*} \in T(w)$.

Lemma 4.3.4. Let $\bar{w}$ be an invariant extension of $w \in \Sigma^{N}$ and let $w^{*} \in T(w)$. Then $\bar{w}$ is an invariant extension of $w^{*}$.

Proof. Let $i, j \in N$. It is sufficient to prove that $\bar{w}$ is an extension of $t_{i, j}(w)$. Suppose not, then by definition of $t_{i, j}$ there must be an $S_{1} \in \mathcal{P}(N)$ with $w_{i}\left(S_{1}\right)=$ 1 , a $j \in S_{1}$, and an $S_{2} \in \mathcal{P}(N)$ with $w_{j}\left(S_{2}\right)=1$, such that $\bar{w}_{i}\left(\left(S_{1} \backslash\{j\}\right) \cup S_{2}\right)=$ 0 . But then, $t_{i, j}(\bar{w})_{i}\left(\left(S_{1} \backslash\{j\}\right) \cup S_{2}\right)=1$ since $\bar{w}$ is an extension of $w$, contradicting invariance of $\bar{w}$.

As announced, in what follows we will show that $T(w)$ contains a unique element, which is also the unique minimal invariant extension of $w$. The next lemma further prepares for this result.

Lemma 4.3.5. Let $w \in \Sigma^{N}$. Then $w$ is invariant if and only if $C^{w}=\left(C^{w}\right)^{*}$.

Proof. For the only-if part, let $t_{i, j}(w)=w$ for all $i, j \in N$ with $i \neq j$. It is sufficient to show that $\left(C^{w}\right)^{r} \subseteq C^{w}$, hence $\left(C^{w}\right)^{r}=C^{w}$, for all $r \geq 1$. We show this by induction over $r$. For $r=1$ this is clear. Now let the claim be true for $r-1$. Let $S \in \mathcal{P}(N)$ and $k \in\left(C^{w}\right)^{r}(S)$. Then by the induction hypothesis

$$
k \in\left(C^{w}\right)^{r}(S)=C^{w}\left(S \cup\left(C^{w}\right)^{r-1}(S)\right)=C^{w}\left(S \cup C^{w}(S)\right) .
$$

Hence, there is a coalition $T \subseteq C^{w}(S) \backslash S$ such that $w_{k}(S \cup T)=1$. If $T=\emptyset$ then $w_{k}(S)=1$, hence $k \in C^{w}(S)$ and we are done. So let $T \neq \emptyset$. Then $w_{\ell}(S)=1$ for all $\ell \in T$. We show that $w_{k}(S)=1$ and, hence, $k \in C^{w}(S)$, by induction over $|T|$. Let $|T|=1$ and $\{\ell\}=T$. Then

$$
\begin{aligned}
w_{k}(S) & =w_{k}(S \cup T \backslash\{\ell\})=w_{k}((S \cup T \backslash\{\ell\}) \cup S) \\
& =t_{k, \ell}(w)_{k}(S \cup T)=w_{k}(S \cup T)=1,
\end{aligned}
$$

where the third equality follows from the definition of $t_{k, \ell}$ and the fourth from invariance of $w$. Hence, $k \in C^{w}(S)$. Let now $m=|T| \geq 2$ and let the claim be true for $m-1$. This implies in particular that $w_{k}(S)=w_{k}(S \cup P)$ whenever $|P|=m-1$. Then let $\ell \in T$ and write $T=T^{\prime} \cup\{\ell\}$, where $\left|T^{\prime}\right|=m-1$. Then

$$
\begin{aligned}
w_{k}(S) & =w_{k}\left(S \cup T^{\prime}\right)=w_{k}(S \cup T \backslash\{\ell\})=w_{k}((S \cup T \backslash\{\ell\}) \cup S) \\
& =t_{k, \ell}(w)_{k}(S \cup T)=w_{k}(S \cup T)=1,
\end{aligned}
$$


where the first equality follows by induction, the third equality again from the definition of $t_{k, \ell}$, and the fourth from invariance of $w$. Hence, $k \in C^{w}(S)$.

For the if-part, let $\left(C^{w}\right)^{*}=C^{w}$. Assume that there are $i, j, k \in N$ with $i \neq j$ and $T \in \mathcal{P}(N)$ such that $t_{i, j}(w)_{k}(T) \neq w_{k}(T)$. Then we must have $i=k$ and $t_{i, j}(w)_{k}(T)=1$ whereas $w_{k}(T)=0$. Hence, there are coalitions $T_{1}$ and $T_{2}$ with $T=\left(T_{1} \backslash\{j\}\right) \cup T_{2}$ such that $w_{k}\left(T_{1}\right)=w_{j}\left(T_{2}\right)=1, j \in T_{1}$, and $w_{k}\left(T_{1} \backslash\{j\}\right)=0$. Hence, $w_{j}(T)=1$, i.e., $j \in C^{w}(T)$, and thus $w_{k}\left(T \cup C^{w}(T)\right)=1$, i.e., $k \in$ $C^{w}\left(T \cup C^{w}(T)\right)=\left(C^{w}\right)^{2}(T)$. On the other hand, $w_{k}(T)=0$ implies $k \notin C^{w}(T)$, a contradiction since $C^{w}(T)=\left(C^{w}\right)^{2}(T)$, as follows from $\left(C^{w}\right)^{*}=C^{w}$.

The preceding results now imply existence of (unique) minimal invariant extensions.

Corollary 4.3.6. Let $w \in \Sigma^{N}$. Then $|T(w)|=1$. If $T(w)=\left\{w^{*}\right\}$, then

1. $C^{w^{*}}=\left(C^{w}\right)^{*}$, and

2. $w^{*}$ is the unique minimal invariant extension of $w$.

Proof. First, let $w^{*} \in T(w)$. We prove that $w^{*}$ satisfies (1.). Therefore, let $S \in$ $\mathcal{P}(N)$. Then $C^{w^{*}}(S)=\left(C^{w^{*}}\right)^{*}(S)$ by Lemma 4.3 .5 , and $\left(C^{w^{*}}\right)^{*}(S)=\left(C^{w}\right)^{*}(S)$ by Corollary 4.3.3, so that $C^{w^{*}}(S)=\left(C^{w}\right)^{*}(S)$. This completes the proof of (1.). Now suppose $w^{*}, w^{\prime} \in T(w)$. Then by (1.), $C^{w^{*}}=C^{w^{\prime}}$, so that $w^{*}=w^{\prime}$. Hence, $|T(w)|=1$, say $T(w)=\left\{w^{*}\right\}$. Let $\bar{w}$ be any invariant extension of $w$, then by Lemma 4.3.4, $\bar{w}$ is an invariant extension of $w^{*}$. This proves (2.).

Thus, applying (in arbitrary order) elementary substitutions to $w \in \Sigma^{N}$ results in the unique minimal invariant extension $w^{*}$. We write $\left(\Sigma^{N}\right)^{*}:=\{w \in$ $\Sigma^{N} \mid w$ is invariant $\}$. The following commuting diagrams summarize the main results of this section and the preceding one.

(i)

$$
\begin{aligned}
& w \in \Sigma^{N} \quad \rightarrow \quad C^{w} \in \Gamma \\
& w^{*} \in \stackrel{\downarrow}{\left(\Sigma^{N}\right)^{*}} \rightarrow C^{w^{*}}=\left(\begin{array}{l}
\downarrow \\
C^{w}
\end{array}\right)^{*} \in \Gamma^{*} \\
& C \in \Gamma \quad \rightarrow \quad w^{C} \in \Sigma^{N}
\end{aligned}
$$

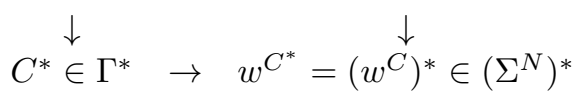

(ii)

\subsection{Power Indices}

In this section we develop a class of power indices for invariant mutual control structures. Of course, such a power index can be applied to an arbitrary mutual 
control structure, simply by applying ot to its unique invariant extension. ${ }^{5}$ As before, the player set is denoted by $N=\{1, \ldots, n\}$, and $\Gamma^{*}$ is the set of all imcs with this player set. A power index is a map $\varphi: \Gamma^{*} \rightarrow \mathbb{R}^{N}$.

For an imcs $C$ the marginal contribution of player $i \in N$ to a coalition $S \subseteq N$ is defined as $\Delta_{i}^{C}(S)=C(S) \backslash C(S \backslash\{i\})$. We say that $i \in N$ is a null player (with respect to $C$ ) if $\Delta_{i}^{C}(S)=\emptyset$ and $i \notin C(S)$ for all $S \subseteq N$. That is, player $i$ is a null player if $i$ is never needed by any coalition to exercise its control, and $i$ is also not controlled by any coalition. The imcs in which every player is a null player, is denoted by $O$, i.e., $O(S)=\emptyset$ for all $S \subseteq N$.

Let $\pi: N \rightarrow N$ be a permutation. Then we define $\pi C \in \Gamma^{*}$ by

$$
(\pi C)(S)=\pi\left(C\left(\pi^{-1}(S)\right)\right) .
$$

The first two axioms that we impose on a power index, are as follows.

Null-Player (NP) $\varphi_{i}(C)=0$ for every null player $i$ with respect to $C$, for every $C \in \Gamma^{*}$.

Constant-Sum (CS) $\sum_{i \in N} \varphi_{i}(C)=\sum_{i \in N} \varphi_{i}(D)$ for all $C, D \in \Gamma^{*}$.

The null-player axiom sets the power of a player who neither controls nor is controlled, equal to 0 . The constant-sum axiom normalizes the power index over different mutual control structures, and plays the role of the usual efficiency axiom. The following observation is almost immediate.

Lemma 4.4.1. Let $\varphi$ be a power index satisfying $N P$ and $C S$. Then $\sum_{i \in N} \varphi_{i}(C)=$ 0 for every $C \in \Gamma^{*}$.

Proof. By NP, $\varphi_{i}(O)=0$ for every $i \in N$. Hence, by CS, $\sum_{i \in N} \varphi_{i}(C)=$ $\sum_{i \in N} \varphi_{i}(O)=0$ for every $C \in \Gamma^{*}$.

The other two basic axioms are the following.

Anonymity $(\mathrm{AN}) \varphi_{\pi(i)}(\pi C)=\varphi_{i}(C)$ for every player $i \in N$, every permutation $\pi$ of $N$, and every $C \in \Gamma^{*}$.

Transfer Property (TP) $\varphi(C)-\varphi\left(C^{\prime}\right)=\varphi(D)-\varphi\left(D^{\prime}\right)$ for all $C, C^{\prime}, D, D^{\prime} \in$ $\Gamma^{*}$ such that $C^{\prime} \subseteq C, D^{\prime} \subseteq D$, and $C(S) \backslash C^{\prime}(S)=D(S) \backslash D^{\prime}(S)$ for every $S \subseteq N$.

\footnotetext{
${ }^{5}$ Attempts to define and characterize power indices directly on the class of all mutual control structures have not been fruitful.
} 
The anonymity axiom needs no further explanation. The transfer property says that if going from $C^{\prime}$ to $C$ involves exactly the same increase in control as going from $D^{\prime}$ to $D$, then the power of each player should change by the same amount when going from $C^{\prime}$ to $C$ as when going from $D^{\prime}$ to $D$. The transfer property is related to a property with the same name, used to characterize the Shapley value (Shapley, 1953) for (monotonic) simple games (Dubey, 1975). The form in which we present it is closely related to a version of the axiom discussed in Dubey et al. (2005). TP is equivalent to a condition closely related to the original format of the transfer axiom as introduced in Dubey (1975).

Lemma 4.4.2. Let $\varphi$ be a power index. Then $\varphi$ satisfies TP if and only if

$$
\varphi(C \cap D)+\varphi(C \cup D)=\varphi(C)+\varphi(D)
$$

for all $C, D \in \mathcal{C}^{*}$ with $C \cup D \in \mathcal{C}^{*}{ }^{6}$

Proof. First, let $\varphi$ satisfy TP and let $C, D \in \Gamma^{*}$ with $C \cup D \in \Gamma^{*}$. Clearly,

$$
(C(S) \cup D(S)) \backslash C(S)=D(S) \backslash(C(S) \cap D(S))
$$

for all $S \subseteq N$. Hence, by TP, $\varphi(C \cup D)-\varphi(C)=\varphi(D)-\varphi(C \cap D)$.

Next, let $\varphi$ satisfy (4.2) for all $C, D \in \mathcal{C}^{*}$ with $C \cup D \in \mathcal{C}^{*}$. We show that $\varphi$ satisfies TP. Let $C, D \in \mathcal{C}^{*}$ such that $C(S) \backslash C^{\prime}(S)=D(S) \backslash D^{\prime}(S)$ for all $S \subseteq N$ and define

$$
E(S)=\bigcup_{T \subseteq S} C(T) \backslash C^{\prime}(T)=\bigcup_{T \subseteq S} D(T) \backslash D^{\prime}(T) .
$$

Clearly, $E \in \mathcal{C}$. Let $p \geq 1$ such that $E^{*}=E^{p}$. If $p=1$ then $E=E^{*} \in \Gamma^{*}$. Suppose $p \geq 2$. If $i \in E^{*}(S)$ then there are coalitions $T_{1}, \ldots, T_{p-1}$ such that

$$
\begin{aligned}
& T_{1} \subseteq E(S), \\
& T_{k} \subseteq E\left(S \cup \bigcup_{l=1}^{k-1} T_{l}\right) \text { for } k=1, \ldots, p-1, \\
& i \in E\left(S \cup \bigcup_{k=1}^{p-1} T_{k}\right) .
\end{aligned}
$$

In particular, we have $T_{1} \subseteq C(S), T_{k} \subseteq C\left(S \cup \bigcup_{l=1}^{k-1} T_{l}\right)$ for $k=1, \ldots, p-1$, and $i \in C\left(S \cup \bigcup_{k=1}^{p-1} T_{k}\right)$. As $C$ is invariant, we have $i \in C(S)$, hence $E^{*}(S) \subseteq C(S)$

\footnotetext{
${ }^{6}$ Note that $C \cap D \in \Gamma^{*}$, see Remark 4.2.11.
} 
for all $S \subseteq N$. Hence, $C \supseteq C^{\prime} \cup E^{*}$. Also, if $j \in C(S)$ and $j \notin C^{\prime}(S)$, then $j \in E(S) \subseteq E^{*}(S)$, so that $C \subseteq C^{\prime} \cup E^{*}$. Thus, $C=C^{\prime} \cup E^{*}$. We show that

$$
C^{\prime}(S) \cap E^{*}(S)=D^{\prime}(S) \cap E^{*}(S)
$$

for all $S \subseteq N$. For this purpose let $i \in C^{\prime}(S) \cap E^{*}(S)$. Then there are $T_{1}, \ldots, T_{p-1}$ as in (4.3). By definition of $E$ there is $R \subseteq S \cup \bigcup_{k=1}^{p-1} T_{k}$ such that $i \in C(R) \backslash C^{\prime}(R)=D(R) \backslash D^{\prime}(R)$. In particular, $i \in D(R)$. We also have $T_{1} \subseteq E(S) \subseteq D(S)$ and $T_{k} \subseteq E\left(S \cup \bigcup_{l=1}^{k-1} T_{l}\right) \subseteq D\left(S \cup \bigcup_{l=1}^{k-1} T_{l}\right)$ so that $i \in D(S)$ by invariance of $D$. Finally, we have $i \in C^{\prime}(S)$ and therefore $i \notin C(S) \backslash C^{\prime}(S)=D(S) \backslash D^{\prime}(S)$. Hence, $i \in D^{\prime}(S)$. Thus, $C^{\prime}(S) \cap E^{*}(S) \subseteq$ $D^{\prime}(S) \cap E^{*}(S)$. The converse inclusion is analogous, so (4.4) holds. As $C^{\prime} \cap E^{*}$ and $D^{\prime} \cap E^{*}$ are invariant by Remark 4.2.11, we find with (4.2) that

$$
\begin{aligned}
\varphi(C)-\varphi\left(C^{\prime}\right) & =\varphi\left(C^{\prime} \cup E^{*}\right)-\varphi\left(C^{\prime}\right) \\
& =\varphi\left(E^{*}\right)-\varphi\left(C^{\prime} \cap E^{*}\right) \\
& =\varphi\left(E^{*}\right)-\varphi\left(D^{\prime} \cap E^{*}\right) \\
& =\varphi\left(D^{\prime} \cup E^{*}\right)-\varphi\left(D^{\prime}\right) \\
& =\varphi(D)-\varphi\left(D^{\prime}\right) .
\end{aligned}
$$

Our main theorem will be a characterization of all power indices satisfying NP, CS, AN, and TP. A crucial result for this is the proposition below, which expresses the power index values assigned to an imcs $C$ as sums of values assigned to so-called unanimity imcs. For every $S \subseteq N$ and $j \in N$ we define the unanimity mutual control structure $U^{S,\{j\}}$ by

$$
U^{S,\{j\}}(T)= \begin{cases}\{j\}, & \text { if } S \subseteq T \\ \emptyset, & \text { otherwise }\end{cases}
$$

It is easy to check that $U^{S,\{j\}}$ is an imcs. Similarly, it is easy to check that $\bigcap_{S \in M} U^{S,\{j\}} \in \Gamma^{*}$ for any subset $M \subseteq \mathcal{P}(N)$ (see also Remark 4.2.11).

Let $C \in \Gamma^{*}$. For a coalition $S \subseteq N$ we define the excess of $S$ by

$$
E^{C}(S)=C(S) \backslash \bigcup_{S^{\prime} \subsetneq S} C\left(S^{\prime}\right) .
$$

Coalition $S$ is minimal controlling in $C$ if $E^{C}(S) \neq \emptyset$. Note that $i \in E^{C}(S)$ if and only if $S$ is a minimal winning coalition in $w_{i}^{C}$. Let $M(C)$ denote the set of minimal controlling coalitions in $C$. 
Example 4.4.3. Consider the invariant mutual control structure from Examples 4.2.9 and 4.3.1 (Porsche-Volkswagen). The minimal controlling coalitions are $\{1\},\{5\},\{1,2,3\}$, and $\{2,3,4\}$. Note that $C^{*}(\{1,5\})=\{4,6\}$, so that both $C^{*}(\{1\})$ and $C^{*}(\{5\})$ are proper subsets of $C^{*}(\{1,5\})$. Still, $\{1,5\}$ is not minimal controlling since $E^{C^{*}}(\{1,5\})=\emptyset$.

We now come to the announced proposition.

Proposition 4.4.4. Let $\varphi$ be a power index satisfying satisfy TP and NP, and let $C \in \Gamma^{*}$. For every $j \in N$ let $M^{j}=\left\{S \in M(C) ; j \in E^{C}(S)\right\}$. Then

$$
\varphi(C)=\sum_{j \in N} \sum_{t=1}^{\left|M^{j}\right|}(-1)^{t+1} \sum_{M \subseteq M^{j},|M|=t} \varphi\left(\bigcap_{S \in M} U^{S,\{j\}}\right) .
$$

Proof. Let $C \in \mathcal{C}^{*}$. Then one easily checks that

$$
C=\bigcup_{S \in M(C)} \bigcup_{j \in E^{C}(S)} U^{S,\{j\}}=\bigcup_{j \in N} \bigcup_{S \in M^{j}} U^{S,\{j\}} .
$$

For each $j \in N$ we define the control structures $C^{j}$ and $C^{-j}$ by

$$
\begin{aligned}
C^{j} & =\bigcup_{S \in M^{j}} U^{S,\{j\}} \\
C^{-j} & =\bigcup_{k \in N \backslash\{j\}} C^{k} .
\end{aligned}
$$

It is easy to see that $C^{j}$ is an invariant mcs. Also $C^{-j}$ is an mcs, and we show that it is invariant, as follows. Let $T \subseteq N$, and suppose $\ell \in N$ with $\ell \notin C^{-j}(T)$. It is sufficient to show that $\ell \notin C^{-j}\left(T \cup C^{-j}(T)\right)$. If $\ell=j$, then clearly $\ell \notin C^{-j}\left(T \cup C^{-j}(T)\right)$. Now suppose $\ell \neq j$. Since $\ell \notin C^{j}(T)$ and $\ell \notin C^{-j}(T)$, we have $\ell \notin C^{j}(T) \cup C^{-j}(T)=C(T)$. Since $C$ is invariant, this implies $\ell \notin C(T \cup C(T))$. Now

$$
\begin{aligned}
C^{-j}\left(T \cup C^{-j}(T)\right) & \subseteq C^{-j}\left(T \cup C^{-j}(T) \cup C^{j}(T)\right) \\
& \subseteq C^{-j}(T \cup C(T)) \cup C^{j}(T \cup C(T)) \\
& =C(T \cup C(T))
\end{aligned}
$$

so that $\ell \notin C^{-j}\left(T \cup C^{-j}(T)\right)$.

By TP and Lemma 4.4.2 we have

$$
\varphi(C)=\varphi\left(C^{-1}\right)+\varphi\left(C^{1}\right)-\varphi\left(C^{-1} \cap C^{1}\right)=\varphi\left(C^{-1}\right)+\varphi\left(C^{1}\right)
$$


where the last equality follows by NP, noting that $C^{-1} \cap C^{1}=O$. Repeating this argument for $C^{-1}, C^{-2}, \ldots$, results in

$$
\varphi(C)=\sum_{j \in N} \varphi\left(C^{j}\right)
$$

Let $j \in N$. It remains to show that

$$
\varphi\left(C^{j}\right)=\sum_{t=1}^{\left|M^{j}\right|}(-1)^{t+1} \sum_{M \subseteq M^{j},|M|=t} \varphi\left(\bigcap_{S \in M} U^{S,\{j\}}\right)
$$

For $\left|M^{j}\right|=1$ there is nothing to show. For $\left|M^{j}\right|=2$, say $M^{j}=\left\{S_{1}, S_{2}\right\}$, we have by TP and Lemma 4.4.2 that

$$
\begin{aligned}
\varphi\left(C^{j}\right) & =\varphi\left(U^{S_{1},\{j\}} \cup U^{S_{2},\{j\}}\right) \\
& =\varphi\left(U^{S_{1},\{j\}}\right)+\varphi\left(U^{S_{2},\{j\}}\right)-\varphi\left(U^{S_{1},\{j\}} \cap U^{S_{2},\{j\}}\right)
\end{aligned}
$$

which results in the desired expression. So suppose $\left|M^{j}\right| \geq 3$ and let $S^{*} \in M^{j}$. By induction we have

$$
\begin{array}{r}
\varphi\left(\bigcup_{S \in M^{j} \backslash\left\{S^{*}\right\}} U^{S,\{j\}} \cap U^{S^{*},\{j\}}\right)=\varphi\left(\bigcup_{S \in M^{j} \backslash\left\{S^{*}\right\}} U^{S \cup S^{*},\{j\}}\right) \\
=\sum_{t=1}^{\left|M^{j}\right|-1}(-1)^{t+1} \sum_{M \subseteq M^{j} \backslash\left\{S^{*}\right\},|M|=t} \varphi\left(\bigcap_{S \in M} U^{S \cup S^{*},\{j\}}\right) \\
=\sum_{t=1}^{\left|M^{j}\right|-1}(-1)^{t+1} \sum_{M \subseteq M^{j} \backslash\left\{S^{*}\right\},|M|=t} \varphi\left(\bigcap_{S \in M \cup\left\{S^{*}\right\}} U^{S,\{j\}}\right) .
\end{array}
$$


Now

$$
\begin{aligned}
\varphi\left(C^{j}\right)= & \varphi\left(\bigcup_{S \in M^{j} \backslash\left\{S^{*}\right\}} U^{S,\{j\}}\right)+\varphi\left(U^{S^{*},\{j\}}\right) \\
& -\varphi\left(\bigcup_{S \in M^{j} \backslash\left\{S^{*}\right\}} U^{S,\{j\}} \cap U^{S^{*},\{j\}}\right) \\
= & \sum_{t=1}^{\left|M^{j}\right|-1}(-1)^{t+1} \sum_{M \subseteq M^{j} \backslash\left\{S^{*}\right\},|M|=t} \varphi\left(\bigcap_{S \in M} U^{S,\{j\}}\right)+\varphi\left(U^{\left.S^{*},\{j\}\right)}\right) \\
= & \sum_{t=1}^{\left|M^{j}\right|}(-1)^{t+1} \sum_{M \subseteq M^{j},|M|=t} \varphi\left(\bigcap_{S \in M} U^{S,\{j\}} \bigcap_{S \in M \cup\left\{S^{*}\right\}} U^{S,\{j\}}\right)
\end{aligned}
$$

where the first and third equalities follow by TP and Lemma 4.4.2, and the second by (4.6) and induction. This completes the proof of the lemma.

Recall that the Shapley-Shubik index (Shapley, 1953; Shapley and Shubik, 1954) of a simple game $w_{i}$ with player set $N$ is defined by

$$
S h_{k}\left(w_{i}\right)=\sum_{S \subseteq N} \frac{(|S|-1) !(|N|-|S|) !}{|N| !}\left(w_{i}(S)-w_{i}(S \backslash\{k\})\right)
$$

for every $k \in N$. Alternatively, as is well-known, the dividends $d_{i}(S)$ of a game $w_{i}$ can be defined, recursively, by

$$
d_{i}(S)= \begin{cases}0, & \text { if } S=\emptyset \\ w_{i}(S)-\sum_{T \nsubseteq S} d_{i}(T), & \text { otherwise }\end{cases}
$$

for all $S \subseteq N$. Then

$$
S h_{k}\left(w_{i}\right)=\sum_{S: k \in S} \frac{d_{i}(S)}{|S|}
$$

for every $k \in N$. For an mcs $C$ and $i \in N$, we write $d_{i}^{C}$ for the dividends of $w_{i}^{C}$. 
For every weight vector $\omega=\left(\alpha_{1}, \ldots, \alpha_{n-1}, \beta_{2}, \ldots, \beta_{n}\right) \in \mathbb{R}^{2 n-2}$, we define the power index $\Phi^{\omega}$ by

$$
\begin{aligned}
\Phi_{i}^{\omega}(C)= & \sum_{k \in N \backslash\{i\}}\left(\sum_{S: i \in S, k \notin S} \frac{d_{k}^{C}(S)}{|S|} \alpha_{|S|}+\sum_{S: i \in S, k \in S} \frac{d_{k}^{C}(S)}{|S|} \beta_{|S|}\right) \\
& -\sum_{k \in N \backslash\{i\}}\left(\sum_{S: i \notin S, k \in S} \frac{d_{i}^{C}(S)}{|S|} \alpha_{|S|}+\sum_{S: i \in S, k \in S} \frac{d_{i}^{C}(S)}{|S|} \beta_{|S|}\right)
\end{aligned}
$$

for all $C \in \Gamma^{*}$ and $i \in N$. The expression in brackets in the first line of (4.7) says that player $i$ receives a kind of weighted Shapley value in the game $w_{k}^{C}$; this expresses the power player $i$ derives from his role in controlling player $k$. The weights depend, both on the size of the coalition of whose dividend player $i$ receives a share, and on whether or not the controlled player $k$ is a member of that coalition. Thus, the first line in (4.7) represents the total power player $i$ acquires from his role in controlling the other players. In the second line, the total (similarly weighted) power that all other players acquire from controlling player $i$, is subtracted.

The central result of this section is the following theorem, in which the class of power indices of the form $\Phi^{\omega}$ is characterized.

Theorem 4.4.5. Let $\varphi$ be a power index. Then $\varphi$ satisfies NP, CS, AN, and $T P$ if and only if there is a weight vector $\omega=\left(\alpha_{1}, \ldots, \alpha_{n-1}, \beta_{2}, \ldots, \beta_{n}\right) \in \mathbb{R}^{2 n-2}$ such that $\varphi(C)=\Phi^{\omega}(C)$ for every $C \in \Gamma^{*}$.

Proof. First, let $\omega=\left(\alpha_{1}, \ldots, \alpha_{n-1}, \beta_{2}, \ldots, \beta_{n}\right) \in \mathbb{R}^{2 n-2}$. We show that $\Phi^{\omega}$ satisfies the four axioms. For AN, this is obvious. When $i$ is a null-player in $C \in \Gamma^{*}$, then $w_{i}^{C}(S)=0$ for every $S$, so $d_{i}^{C}(S)=0$ for every $S$. Moreover, by using the definition of a dividend it is easy to show that $d_{k}^{C}(S)=0$ for every $S$ with $i \in S$. Hence, $\Phi_{i}^{\omega}(C)=0$, which shows NP. CS, more specifically $\sum_{i \in N} \Phi_{i}^{\omega}(C)=0$ for every $C \in \Gamma^{*}$, follows almost directly from the definition of $\Phi^{\omega}$. Finally, for $\mathrm{TP}$, let $C^{\prime} \subseteq C$ and $D^{\prime} \subseteq D$ satisfy $C(S) \backslash C^{\prime}(S)=D(S) \backslash D^{\prime}(S)$ for every $S$. Then it is easily verified that for every player $j$ and every coalition $S$, we have $d_{j}^{C}(S)-d_{j}^{C^{\prime}}(S)=d_{j}^{D}(S)-d_{j}^{D^{\prime}}(S)$. Hence,

$$
\begin{aligned}
& \Phi^{\omega}(C)-\Phi^{\omega}\left(C^{\prime}\right)= \\
& \sum_{k \in N \backslash\{i\}}\left(\sum_{S: i \in S, k \notin S} \frac{d_{k}^{C}(S)-d_{k}^{C^{\prime}}(S)}{|S|} \alpha_{|S|}+\sum_{S: i \in S, k \in S} \frac{d_{k}^{C}(S)-d_{k}^{C^{\prime}}(S)}{|S|} \beta_{|S|}\right)
\end{aligned}
$$




$$
\begin{aligned}
& -\sum_{k \in N \backslash\{i\}}\left(\sum_{S: i \notin S, k \in S} \frac{d_{i}^{C}(S)-d_{i}^{C^{\prime}} k(S)}{|S|} \alpha_{|S|}+\sum_{S: i \in S, k \in S} \frac{d_{i}^{C}(S)-d_{i}^{C^{\prime}}(S)}{|S|} \beta_{|S|}\right) \\
& =\sum_{k \in N \backslash\{i\}}\left(\sum_{S: i \in S, k \notin S} \frac{d_{k}^{D}(S)-d_{k}^{D^{\prime}}(S)}{|S|} \alpha_{|S|}+\sum_{S: i \in S, k \in S} \frac{d_{k}^{D}(S)-d_{k}^{D^{\prime}}(S)}{|S|} \beta_{|S|}\right) \\
& -\sum_{k \in N \backslash\{i\}}\left(\sum_{S: i \notin S, k \in S} \frac{d_{i}^{D}(S)-d_{i}^{D^{\prime}} k(S)}{|S|} \alpha_{|S|}+\sum_{S: i \in S, k \in S} \frac{d_{i}^{D}(S)-d_{i}^{D^{\prime}}(S)}{|S|} \beta_{|S|}\right) \\
& =\Phi^{\omega}(D)-\Phi^{\omega}\left(D^{\prime}\right)
\end{aligned}
$$

which shows TP.

For the converse, let $\varphi$ be a power index satisfying the four axioms. By NP, CS, AN, and Lemma 4.4.1 there are numbers $\alpha_{|S|}$ for $|S|=1, \ldots, n-1$ and $\beta_{|S|}$ for $|S|=2, \ldots, n$ such that for every $\emptyset \neq S \subseteq N$ and $j \in N$ we have

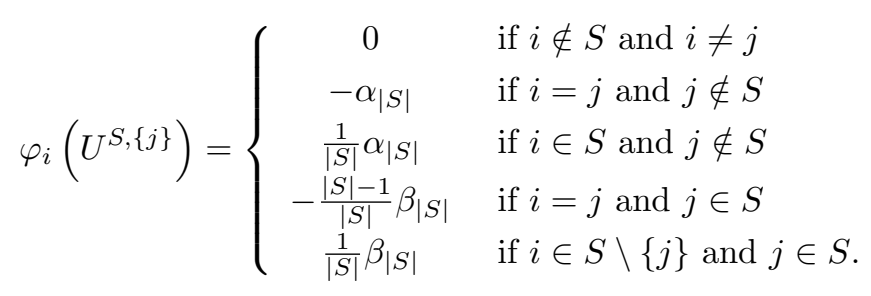

By Proposition 4.4.4 and the first part of the proof it is sufficient to prove that with $\omega=\left(\alpha_{1}, \ldots, \alpha_{n-1}, \beta_{2}, \ldots, \beta_{n}\right) \in \mathbb{R}^{2 n-2},(4.8)$ holds for $\Phi^{\omega}$ as well. Let $w$ be the vector of simple games associated with the unanimity control structure $U^{S,\{j\}}$. Then $d_{i}(T)=0$ for every $i \neq j$ and every $T \subseteq N$, whereas $d_{j}(T)=1$ for $T=S$ and $d_{j}(T)=0$ otherwise. It is now straightforward to verify that $\Phi^{\omega}$ satisfies (4.8).

The family of power indices in Theorem 4.4.5 is quite large, since the axioms put no restrictions whatsoever on the values of the weights in $\omega$. The following very natural condition on a power index $\varphi$ results in nonnegativity of the weights.

Monotonicity (MO) $\varphi_{i}(C) \geq \varphi_{i}(D)$ for all $C, D \in \Gamma^{*}$ and $i \in N$ such that (i) $i \in C(S) \Rightarrow i \in D(S)$ and (ii) $\Delta_{i}^{D}(S) \subseteq \Delta_{i}^{C}(S)$ for all $S \subseteq N$.

Corollary 4.4.6. Let $\varphi$ be a power index. Then $\varphi$ satisfies NP, CS, AN, TP, and $M O$ if and only if there is a weight vector $\omega=\left(\alpha_{1}, \ldots, \alpha_{n-1}, \beta_{2}, \ldots, \beta_{n}\right) \in \mathbb{R}_{+}^{2 n-2}$ such that $\varphi(C)=\Phi^{\omega}(C)$ for every $C \in \Gamma^{*}$. 
Proof. We leave it to the reader to verify that $\Phi^{\omega}$ satisfies MO if $\omega \in \mathbb{R}_{+}^{2 n-2}$. Conversely, let $\omega=\left(\alpha_{1}, \ldots, \alpha_{n-1}, \beta_{2}, \ldots, \beta_{n}\right) \in \mathbb{R}^{2 n-2}$ and suppose $\Phi^{\omega}$ is monotonic. If $S \subseteq N, i \in S$ and $j \notin S$, then by MO we have $\Phi_{i}^{\omega}\left(U^{S,\{j\}}\right) \geq \Phi_{i}^{\omega}(O)$, hence, $\alpha_{|S|} /|S| \geq 0$. If $S \subseteq N, i \in S \backslash\{j\}$ and $j \in S$ then again by MO we have $\Phi_{i}^{\omega}\left(U^{S,\{j\}}\right) \geq \Phi_{i}^{\omega}(O)$, hence $\beta_{|S|} /|S| \geq 0$.

A complete determination of the weights is obtained by replacing MO by the following condition.

Controlled player (CP) For all $C \in \Gamma^{*}, j \in C(N)$, and $i \in N \backslash C(N)$,

$$
\varphi_{j}(C)= \begin{cases}-1, & \text { if } \Delta_{j}^{C}(S)=\emptyset \text { for all } S \subseteq N \\ \varphi_{i}(C)-1, & \text { if } \Delta_{i}^{C}(S)=\Delta_{j}^{C}(S) \text { for all } S \subseteq N .\end{cases}
$$

The controlled player condition says that if $j$ is a 'controlled player', i.e., controlled by at least one coalition and, thus, by $N$, but does not himself exercise any control, then the power of $j$ is fixed at -1 . Also, if $i$ is an uncontrolled player, i.e., controlled by no coalition at all, but $i$ and $j$ exercise exactly the same control, then their difference in power is fixed at 1 , that is, $i$ gets assigned 1 more than $j$. Note that, if $\varphi$ also satisfies NP and $i$ is a null player, then the second consequence in CP implies the first. We now have the following corollary. Its proof follows again easily from examining the unanimity mutual control structures of the form $U^{S,\{j\}}$, and is left to the reader.

Corollary 4.4.7. Let $\varphi$ be a power index. Then $\varphi$ satisfies $N P, C S, A N, T P$, and $C P$ if and only if $\varphi(C)=\Phi^{\omega}(C)$ for every $C \in \Gamma^{*}$, where $\omega=(1, \ldots, 1) \in \mathbb{R}^{2 n-2}$.

We apply this unique power index to the Porsche-Volkswagen case.

Example 4.4.8. Consider the invariant mutual control structure $C^{*}$ from Example 4.2.9. Then we find for $\omega=(1, \ldots, 1) \in \mathbb{R}^{2 n-2}$ that $\Phi_{1}^{\omega}\left(C^{*}\right)=\frac{14}{12}$, $\Phi_{2}^{\omega}\left(C^{*}\right)=\Phi_{3}^{\omega}\left(C^{*}\right)=\frac{7}{12}, \Phi_{4}^{\omega}\left(C^{*}\right)=-\frac{5}{6}, \Phi_{5}^{\omega}\left(C^{*}\right)=-\frac{1}{2}$, and $\Phi_{6}^{\omega}\left(C^{*}\right)=-1$. It is interesting to compare the power of Porsche Families with its power at the end of 2007. Figure 2 depicts the control structure between the same companies at the end of 2007. At that time, Volkswagen was not controlled by any group of main investors. Although Porsche SE has veto power in the game on Volkswagen AG, we ignore this fact, as it is not clear how this power can be exercised. We describe this situation by a mutual control structure $D$. Its minimal invariant extension is:

$$
D^{*}(S)=\{4,6\} \text { for all } S \text { with } 1 \in S .
$$

Thus, even while ignoring the power of Porsche Families on Volkswagen, we still have $\Phi_{1}^{\omega}\left(D^{*}\right)=2>\frac{14}{12}=\Phi_{1}^{\omega}\left(C^{*}\right)$. Hence, according to this power index it had more power in 2007 than it has now. 


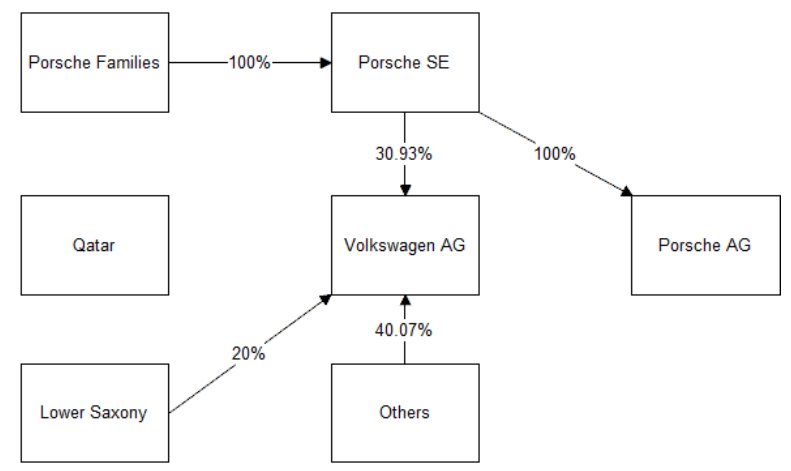

Figure 2: Porsche and VW voting rights by the end of 2007, based on the 2007 annual report of Volkswagen AG and the 2007/2008 annual report of Porsche Automobil Holding SE GmbH.

Note that case of $\omega=(1, \ldots, 1) \in \mathbb{R}^{2 n-1}$ we have that

$$
\Phi_{i}(C)=\sum_{k \neq i} S h_{i}\left(w_{k}^{C}\right)-\sum_{k \neq i} S h_{k}\left(w_{i}^{C}\right) .
$$

Hence, player $i$ obtains the sum of all his Shapley-Shubik values in the games in which he contributes to controlling the other players, minus the sum of all Shapley-Shubik values of the other players in the game describing the control undergone by $i$.

We conclude this section with, first, showing the logical independence of the axioms in Theorem 4.4.5 and, second, discussing a variation with a variable number of players..

\subsubsection{Independence of the Axioms}

We show the independence of the acioms in Theorem 4.4.5.

Null-Player Fix $\varepsilon>0$ and for every nonempty $S \subseteq N$ and $j \in N$ define

$$
\varphi_{i}\left(U ^ { S , \{ j \} ) } \left\{\begin{array}{cl}
\varepsilon & \text { if } i \notin S \text { and } i \neq j \\
-1 & \text { if } i=j \text { and } j \notin S \\
\frac{1}{|S|}-\frac{|N|-|S|-1}{|S|} \varepsilon & \text { if } i \in S \text { and } j \notin S \\
-\frac{|S|-1}{|S|} & \text { if } i=j \text { and } j \in S \\
\frac{1}{|S|}-\frac{|N|-|S|}{|S|-1} \varepsilon & \text { if } i \in S \backslash\{j\} \text { and } j \in S .
\end{array}\right.\right.
$$


Extend $\varphi$ to $\Gamma^{*}$ by using Proposition 4.4.4. Then $\varphi$ satisfies CS, AN, and TP, but not NP.

Constant Sum Define the power index $\varphi$ by $\varphi_{i}(C)=|C(\{i\})|-w_{i}^{C}(N)$ for all $C \in \Gamma^{*}$ and $i \in N$. Then $\varphi$ satisfies NP and AN. Also, for $C, D \in \Gamma^{*}$,

$$
\begin{aligned}
\phi_{i}(C \cup D)+\phi_{i}(C \cap D)= & |(C(\{i\}) \cup D(\{i\}))|-w_{i}^{C \cup D}(N) \\
& +|(C(\{i\}) \cap D(\{i\}))|-w_{i}^{C \cap D}(N) \\
= & |C(\{i\})|+|D(\{i\})|-\left(w_{i}^{C}(N)+w_{i}^{D}(N)\right) \\
= & \phi_{i}(C)+\phi_{i}(D)
\end{aligned}
$$

for all $i \in N$, so that $\varphi$ satisfies TP by Lemma 4.4.2. Consider $D \in \Gamma^{*}$ defined by $D(S)=N$ for all nonempty $S \subseteq N$. Then $\varphi_{i}(D)=|N|-1$ for all $i \in N$, so that $\varphi$ cannot satisfy CS by Lemma 4.4.1.

Anonymity Let $N=\{1,2\}$ and define $\varphi$ by $\varphi\left(U^{\{1\},\{1\}}\right)=\varphi\left(U^{\{2\},\{2\}}\right)=$ $\varphi\left(U^{\{2\},\{1\}}\right)=(0,0), \varphi\left(U^{\{1,2\},\{2\}}\right)=-\varphi\left(U^{\{1,2\},\{1\}}\right)=\varphi\left(U^{\{1\},\{2\}}\right)=(1,-1) ;$ and by extending $\varphi$ to $\Gamma^{*}$ using Proposition 4.4.4. Then $\varphi$ satisfies NP, CS, and $\mathrm{TP}$, but not AN.

Transfer property Recall the normalized Banzhaf index (cf. Banzhaf, 1965; Dubey et al., 2005)

$$
B z_{i}(v)= \begin{cases}\frac{\mu_{i}(v)}{\sum_{j \in N} \mu_{j}(v)}, & \text { if } \mu_{i} \neq 0 \\ 0, & \text { otherwise }\end{cases}
$$

where $\mu_{i}(v)$ is the number of coalitions $S \subseteq N$ in which player $i$ is pivotal with respect to $v$. Define the power index $\varphi$ by

$$
\varphi_{i}(C)=\sum_{j \neq i} B z_{i}\left(w_{j}^{C}\right)-\sum_{j \neq i} B z_{j}\left(w_{i}^{C}\right)
$$

for all $C \in \Gamma^{*}$ and $i \in N$. It is straightforward to verify that $\varphi$ satisfies NP, $\mathrm{CS}$, and AN. We show that it does not satisfy TP by using Lemma 4.4.2. For $S, S^{\prime} \subseteq N$ define $U^{S, S^{\prime}} \in \Gamma^{*}$ by $U^{S, S^{\prime}}(T)=S^{\prime}$ if $S \subseteq T$ and $U^{S, S^{\prime}}(T)=\emptyset$ otherwise. Now take $N=\{1,2,3\}$ and let $C=U^{\{1,2\}, N}$ and $D=U^{\{1,3\}, N}$. Then $B z_{1}\left(w_{i}^{C}\right)=\frac{1}{2}$ and $B z_{1}\left(w_{i}^{D}\right)=\frac{1}{2}$ for $i=1,2,3$. Hence, $\varphi_{1}(C)=\frac{1}{2}$. Further,

$$
\begin{aligned}
& C \cup D=U^{\{1,2\}, N} \cup U^{\{1,3\}, N}, \\
& C \cap D=U^{N, N} .
\end{aligned}
$$


Now $B z_{1}\left(w_{i}^{C \cup D}\right)=\frac{3}{5}$ for $i=1,2,3$. Hence, $\varphi_{1}(C \cup D)=\frac{4}{5}$. Further, by computation or by $\mathrm{CS}$ and $\mathrm{AN}$, we have $\varphi(C \cap D)=0$. Thus,

$$
\varphi_{1}(C \cup D)+\varphi_{1}(C \cap D)=\frac{4}{5} \neq 1=\varphi_{1}(C)+\varphi_{1}(D) .
$$

So, $\varphi$ does not satisfy TP.

\subsubsection{Variable number of players}

In view of the area of application, it is not unnatural to assume that the number of players can vary. New firms or investment companies may enter the scene, while existing players may disappear. In the Porsche-Volkswagen case, we may choose to regard the small shareholders as a null-player, or simply disregard them, as we did above. In the same case, as noted before, at mid June 2013 Qatar left the scene.

To accommodate this, let a power index $\varphi$ now be defined for every invariant mutual control structure $(N, C)$, where $N$ is an arbitrary but finite set of players, say $N \subseteq \mathbb{N}$ with $|N|<\infty$. We concentrate on a modification of Corollary 4.4.7 about the power index with all weights equal to one, but the other characterization results above can be modified in a similar fashion.

The axioms CS, AN, and TP are basically the same as before; we now call them $\mathrm{CS}^{*}, \mathrm{AN}^{*}$, and $\mathrm{TP}^{*}$. We modify $\mathrm{NP}$ and $\mathrm{CP}$ to $\mathrm{NP}^{*}$ and $\mathrm{CP}^{*}$, as follows.

Null-Player* $\left(\mathbf{N P}^{*}\right) \varphi_{i}(N, C)=0$ and $\varphi_{j}\left(N \backslash\{i\}, C^{\prime}\right)=\varphi_{j}(N, C)$ for every null player $i$ in $(N, C)$ and every player $j \in N \backslash\{i\}$, where $C^{\prime}(S)=C(S)$ for every $S \subseteq N \backslash\{i\}$, for every invariant mutual control structure $(N, C)$.

Controlled Player* $\left(\mathbf{C P}^{*}\right)$ For every invariant mutual control structure

$(N, C)$, every $j \in C(N)$, and every $i \in N \backslash C(N)$, if $\Delta_{i}^{C}(S)=\Delta_{j}^{C}(S)$ for all $S \subseteq N$, then $\varphi_{j}(C)=\varphi_{i}(C)-1$.

The modified null-player axiom $\mathrm{NP}^{*}$ not only says that null-players have zero power, but also that null-players can be left out (or added, for that matter) without affecting the power of the other players. Note that this axiom establishes a link between the values of the power index for mutual control structures with different player sets.

Compared to $\mathrm{CP}$, the $\mathrm{CP}^{*}$ version is simpler: the omitted part of $\mathrm{CP}$ can be obtained by adding a null-player. The following result can be derived almost immediately from Corollary 4.4.7 - we omit the proof.

Corollary 4.4.9. Let the number of players be variable. There is a unique power index $\Phi^{*}$ satisfying $N P^{*}, C S^{*}, A N^{*}, T P^{*}$, and $C P^{*}$. For every invariant mutual control structure $(N, C)$, we have $\Phi^{*}(N, C)=\Phi^{\omega}(N, C)$, where $\omega=(1, \ldots, 1) \in$ $\mathbb{R}^{2|N|-2}$. 


\subsection{Conclusion}

We have introduced a new model to represent mutual control within a set of players. Our model can be understood as a collection of simple games, one for each player. A similar model has been introduced earlier by Gambarelli and Owen (1994) where a set of investors controls a set of firms. However, our model is much more general: Investors in the article of Gambarelli and Owen (1994) are simply uncontrolled players in our model.

Crama and Leruth (2011) point out that the concepts of control and ownership must be carefully distinguished. The following example emphasizes this claim.

Example 4.5.1. Let $a, b, c, d$ be firms such that $a$ owns $80 \%$ of the shares of $b$ and $c$ owns the remaining $20 \%$ of $b$. Let further $a$ 's shares distributed such that $c$ owns $40 \%$ and $d$ owns $60 \%$. See the following diagram.

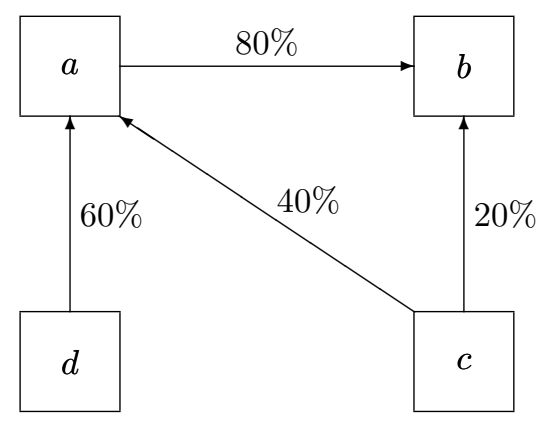

Assume that all decisions can be made by an absolute majority. From a financial point of view $c$ owns $32 \%$ of $b$ indirectly via $a$ and $20 \%$ directly, so all together $52 \%$ which is the majority. On the other hand $d$ owns $48 \%$ of $b$ indirectly via $a$. However, if we build a mutual control structure $C$ to represent this situation, we find $C(\{a\})=\{b\}, C(\{d\})=\{a\}$ and $C(\{c\})=\emptyset$. For the minimal invariant extension of $C$ we have $C^{*}(\{d\})=\{a, b\}$ and $C^{*}(\{c\})=\emptyset$. We see that indirect ownership of firms might not lead to any control at all. In particular, while indirect ownership is proportional to the number of shares, indirect control follows a winner takes all principle: Although firm c owns $52 \%$ of firm $b$ its indirect votes are useless as it can not force firm $a$ to use it.

As we pointed out in the introduction, our theory can be used to evaluate control structures, provided we can model them as a mutual control structure (i.e., an element of $\mathcal{C}$ ). This modelling part is important and should be done with care, as the following example illustrates.

Example 4.5.2. Consider five players, $N=\{1, \ldots, 5\}$, and a mutual control structure $C$ determined by $C(\{1\})=\{2\}$ and $C(\{2\})=\{3,4,5\}$. Then $C^{*}$ is 
determined by $C^{*}(\{1\})=\{2,3,4,5\}$ and $C^{*}(\{2\})=\{3,4,5\}$. One might argue here that player 2 should actually be left out. Indeed, if this player is merely an administrative entity (a mailbox, or an office), then it would be natural to leave it out and consider the mutual control structure $C^{\prime}$ with $C^{\prime}(\{1\})=\{3,4,5\}$ instead of $C$, and thus with player 2 as a null-player. If, however, player 2 is a real player, then it should be left in. In that case, one might argue that a mutual control structure $C^{\prime \prime}$ with $C^{\prime \prime}(\{1\})=\{2,3,4,5\}$ and $C^{\prime \prime}(\{2\})=\emptyset$ would reflect the situation more adequately than $C^{*}$, but this is, indeed, subject to discussion: player 2 would be put at the same level of control as players 3,4 , and 5 , which is questionable. Applying the power index $\varphi$ with $\varphi=\Phi^{\omega}, \omega=(1, \ldots, 1) \in \mathbb{R}^{8}$, one obtains

$$
\begin{aligned}
\varphi\left(C^{*}\right) & =(5 / 2,1 / 2,-1,-1,-1) \\
\varphi\left(C^{\prime}\right) & =(3,0,-1,-1,-1) \\
\varphi\left(C^{\prime \prime}\right) & =(4,-1,-1,-1,-1)
\end{aligned}
$$

reflecting each of the three choices above. In favor of $C^{*}$ one could argue that, although player 2 is completely controlled by player 1 , the fact that he has direct control over 3,4 , and 5 , is reflected by its positive power index which, moreover, would increase if the number of players it directly controls, increases. In general, it seems doubtful whether it is possible to find an updating procedure, leading to 'invariant' mutual control structures, that would adequately cover each and every possible situation. In applications, the modelling phase, resulting in a formal mutual control structure $C$, should therefore be done with care.

There are several interesting avenues for further theoretical research in this area. One obvious question is what becomes feasible in terms of power indices if we change the axioms. Section 4.4.1 already considers some possibilities, e.g., a Banzhaf value based power index becomes possible if the Transfer Property is dropped. Another interesting direction is to consider the combination of mutual control structures with transferable utility games in the spirit of Myerson (1977). A mutual control structure can be seen as a hypergraph, and a value for transferable utility games combined with such a hypergraph can take the imposed control relations into consideration. 


\section{Bibliography}

Arrow, K., 1951. Social Choice and Individual Values. Cowles Foundation for Research in Economics at Yale University.

Aumann, R., Drèze, J., 1974. Cooperative games with coalition structures. International Journal of Game Theory 3, 217-237.

Aumann, R., Maschler, M., 1964. The Bargaining Set for Cooperative Games, in: Dresher, M., Shapley, L., Tucker, A. (Eds.), Advances in Game Theory. Princeton University Press, pp. 443-476.

Aumann, R., Maschler, M., 1985. Game Theoretic Analysis of a Bankruptcy Problem from the Talmud. Journal of Economic Theory 36, 195-213.

Aumann, R., Myerson, R., 1988. Endogenous Formation of Links Between Players and of Coalitions: An Application of the Shapley Value, in: Roth (Ed.), The Shapley value, Essays in Honor of Lloyd Shapley. Cambridge University Press, pp. 175-191.

Axelrod, R. (Ed.), 1970. Conflict of Interests: A Theory of Divergent Goals with Application to Politics. Markham Publishing Company.

Banerjee, S., H., K., Sönmez, T., 2001. Core in a Simple Coalition Formation Game. Social Choice and Welfare 19, 135-153.

Banzhaf, J., 1965. Weighted Voting Doesn't Work: A Mathematical Analysis. Rutgers Law Review 19, 317-343.

Black, D., 1948. On the Rationale of Group Decision-making. Journal of Political Economy 56, 23-34.

Bloch, F., Dutta, B., 2011. Formation of Networks and Coalition, in: Benhabib, J., Bisin, A., Jackson, M. (Eds.), Handbook of Social Economics. Elsevier, pp. 729-779. 
Bogomolnaia, A., Jackson, M., 2002. The Stability of Hedonic Coalition Structurs. Games and Economic Behavior 38, 201-230.

Bondareva, O., 1963. Some Application of Linear Programming Methods to the Theory of Cooperative Games. Problemi Kibernitiki 10, 119-139.

Casajus, A., 2009. Outside Options, Component Efficiency, and Stability. Games and Economics Behavior 65, 49-61.

Chun, Y., Thomson, W., 1992. Bargaining Problems with Claims. Mathematical Social Science 24, 19-33.

de Clippel, G. Serrano, S., 2008. Marginal Contributions and Externalities in the Value. Econometrica 76, 1413-1436.

Coleman, J., 1971. Control of Collectives and the Power of a Collectivity to Act, in: Liebermann, A. (Ed.), Social Choice. Gordon and Breach, pp. 192-225.

Crama, Y., Leruth, L., 2007. Control and Voting Power in Corporate Networks: Concepts and Computational Aspects. European Journal of Operations Research 178, 879-893.

Crama, Y., Leruth, L., 2011. Power Indices and the Measurement of Control in Corporate Structures. International Game Theory Review, forthcoming.

Curiel, I., Maschler, M., Tijs, S., 1987. Bankruptcy Games. Zeitschrift für Operations Research 31, 143-159.

Demange, G., 1994. Intermediate preferences and stable coalition structures. Journal of Mathematical Economics 23, 45-58.

Dimitrov, D., Haake, C., 2006. Government versus Opposition: Who Should be Who in the 16th German Bundestag. Journal of Economics 89, 115-128.

Dimitrov, D., Haake, C., 2008a. A Note on the Paradox of Smaller Coalitions. Social Choice and Welfare 30, 571-579.

Dimitrov, D., Haake, C., 2008b. Stable Governments and the Semistrict Core. Games and Economic Behavior 62, 460-475.

Drèze, J., Greenberg, J., 1980. Hedonic Coalitions: Optimalilty and Stability. Econometrica 48, 987-1003.

Dubey, P., 1975. On the Uniqueness of the Shapley Value. International Journal of Game Theory 4, 131-139. 
Dubey, P., Einy, E., Haimanko, O., 2005. Compound Voting and the Banzhaf Index. Games and Economic Behavior 51, 20-30.

Dubey, P., Shapley, L., 1979. Mathematical Properties of the Banzhaf Power Index. Mathematics of Operations Research 4, 99-131.

Dummet, M., Farquharson, R., 1961. Stability in Voting. Econometrica 29, 33-43.

Dutta, B., Ehlers, L., Kar, A., 2010. Externalities, potential, value, and consistency. Journal of Economic Theory 145, 2380-2411.

Einy, E., 1985. On connected coalitions in dominated simple games. International Journal of Game Theory 14, 103-125.

Einy, E., Wettstein, D., 1996. Equivalence Between Bargaining Sets and the Core in Simple Games. International Journal of Game Theory 25, 65-71.

Gambarelli, G., Owen, G., 1994. Indirect Control of Corporations. International Journal of Game Theory 23, 287-302.

Gillies, D., 1959. Solutions to general non-zero games, in: Kuhn, A., Luce, R. (Eds.), Contributions to the Theory of Games. Princeton University Press, pp. 47-85.

Greenberg, J., 1992. On the Sensitivity of von Neumann and Morgenstern Stable Sets: The Stable and the Individually Stable Bargaining Set. International Journal of Game Theory 21, 41-55.

Greenberg, J., Weber, S., 1986. Strong tiebout equilibrium under restricted preferences domain. Journal of Economic Theory 38, 101-117.

Hart, S., Kurz, M., 1983. Endogenous Formation of Coalitions. Econometrica 51, 1047-1064.

Hart, S., Kurz, M., 1984. Stable Coalition Structures, in: Holler, M. (Ed.), Coalitions and Collective Actions. Physica Verlag, pp. 235-258.

Iehlé, V., 2007. The Core-Partition of Hedonic Games. Mathematical Social Sciences 54, 176-185.

Kalai, E., 1977. Proportional Solutions to Bargaining Situations: Interpersonal Utility Comparisons. Econometrica 45, 1623-1630.

Karos, D., 2012. Coalition Formation in General Apex Games. FEEM Working Paper No. 38.2012. 
Karos, D., 2013. Bargaining and Power. FEEM Working Paper No. 63.2013.

Karos, D., Peters, H., 2013. Mutual Control Structures. Working Paper.

Mas-Colell, A., 1989. An equivalence theorem for a bargaining set. Journal of Mathematical Economics 18, 129-139.

Milchtaich, I., Winter, E., 2002. Stability and segregation in group formation. Games and Economic Behavior 38, 318-346.

Montero, M., 2002. Non-Cooperative Bargaining in Apex Games and the Kernel. Games and Economic Behavior 41, 309-321.

Nash, J., 1950. The Bargaining Problem. Econometrica 18, 155-162.

von Neumann, J., Morgenstern, O., 1944. Theory of Games and Economic Behaviour. Princeton University Press.

Owen, G., 1977. Values of Games with A Priori Unions, in: Hein, R., Moeschlin, O. (Eds.), Essays in Mathematical Economics and Game Theory. Springer, pp. $76-88$.

Owen, G., 1978. Characterization of the Banzhaf-Coleman Index. SIAM Journal on Applied Mathematics 35, 315-327.

Peleg, B., 1981. Coalition Formation in Simple Games with Dominant Players. International Journal of Game Theory 10, 11-33.

Penrose, L., 1946. The Elementary Statistics of Majority Voting. Journal of the Royal Statistical Society 109, 53-57.

Riker, W., 1962. The Theory of Political Coalitions. Yale University Press.

Sagonti, E., 1991. On the Strong Monotonicity of Power Indices. International Journal of Game Theory 20, 13-22.

Shapley, L., 1953. A Value for n-Person Games, in: Kuhn, H., Tucker, A. (Eds.), Contributions to the Theory of Games. Princeton University Press, pp. 307317.

Shapley, L., 1954. Simple Games: An Outline of the Descriptive Theory. RAND Corporation Research Memorandum RM-1384.

Shapley, L., 1962. Simple Games: An Outline of the Descriptive Theory. Behavioral Science 7, 59-66. 
Shapley, L., 1967. On Balanced Sets and the Core. Naval Research Logistics Quarterly 14, 453-460.

Shapley, L., Shubik, M., 1954. A Method for Evaluating the Distribution of Power in a Committee System. The American Political Science Review 48, 787-792.

Shenoy, P., 1979. On Coalition Formation: A Game-Theoretical Approach. International Journal of Game Theory 8, 133-164.

de Swaan, A., 1973. Coalition Theories and Cabinet Formation. Elsevier Scientific Publishing Company.

Young, H., 1985. Monotonic Solutions of Cooperative Games. International Journal of Game Theory 14, 65-72. 



\section{Index}

alternatives, 9

set of, 11,12

anonymity, see power index

apex game, 12, 16, 18, 36, 39, 52, 53,

55,59

general, 16, 19, 23, 24, 28, 29, 31, 33-35

apex player, see player

apex quota, see quota

apex set, 16, 19

balancedness

ordinal, 21

pivot, 21

bankruptcy problem, 41

Banzhaf index, 16, 28, 29, 37

normalized, 16, 32, 34-36, 85

Banzhaf-Coleman index, see Banzhaf index

bargaining problem, 39, 40

with claims, 41

bargaining rule, 39

bargaining set

Aumann-Maschler, 11

individual stable, 11

Mas-Colell, 11

bargaining solution, 40

constrained egalitarian, 43, 45, 61

continuous, $\mathbf{4 2}, 43$

efficient, 42, 43, 46

egalitarian, 42, 45, 54, 55, 59, 61

fair, $\mathbf{4 2}, 43,51$

individually rational, $\mathbf{4 2}$, 43, 46, 51 proportional, 43, 47, 52, 53

symmetric, 42, 46, 47

Bundestag, 38, 60

cabinet, 38,61

Casajus value, 37

coalition, 9, 13, 63

blocking, 21, 52

core stable, 16, 17, 22, 22, 23, 24, $26,27,29,31,35,36, \mathbf{5 2}, 53$, 54,59

formation, 11, 13, 21, 37

individually stable, $\mathbf{5 1}, 54$

infeasible, 39, 60

internally stable, $39,51, \mathbf{5 1}$

minimal controlling, $\mathbf{7 7}$

minimal winning, 13, 77

Nash stable, $39, \mathbf{5 0}, 51,54$

null player free, $\mathbf{1 7}, 22,24,26,35$

restriction, $\mathbf{6 0}$

winning, $9, \mathbf{1 3}, 28,40$

committee, 9, 12, 37

consecutiveness, 21

constrained egalitarian bargaining solution, see bargaining solution

control, $63,65,75,87$

indirect, $63, \mathbf{6 6}, 68,71$

core

of a hedonic game, 21, 22, 24, 26, $29,31,36$

of a TU game, 10

core stable coalition, see coalition

core stable partition, see partition 
corporate finance, 13

corporate governance, 63

deviation, 21, $5 \mathbf{2}$

disagreement point, $\mathbf{4 0}, 41$

dividend, $65,80,80$

drug market, 27

efficient power index, see power index

egalitarian bargaining solution, see bargaining solution

elementary substitution, $\mathbf{7 1}, 72$

invariant under, $\mathbf{7 2}$

equal treatment property, see power index

excess, $7 \mathbf{7}$

executive board, 18

fixed point, 43

general apex game, see apex game grand coalition, 11, 30

hedonic game, 11, 15, 16, 21, 50, 52

with externalities, 62

imcs, see mutual control structure

indirect control, see control

information, 27

invariant mutual control structure, see mutual control structure

investor, 13,87

marginal contribution, 38, 40

in mutual control structures, $\mathbf{7 5}$

mcs, see mutual control structure

minimal invariant extension, see invariant extension

minimal winning coalition, see coalition

minor player, see player

minor quota, see quota

monotonic simple game, see simple game monotonicity of a simple game, see simple game strong, see power index

mutual control structure, 63, 65, 69 associated simple games, 70

intersection, 70

invariant, 63, 66, 68, 69, 75

invariant extension

minimal, 63, 64

monotonic, 63, 65

transitive, $\mathbf{6 7}$

unanimity, $\mathbf{7 7}$

union, 70

normalized Banzhaf index, see Banzhaf index

null player, see player

null player free coalition, see coalition

null player property, see power index

opportunity costs, 62

ordinal balancedness, see balancedness

outside option, $39, \mathbf{4 0}$

Owen value, 37

ownership, 87

partition, 13, 21

blocked, 21

core stable, 11, 12, 21, 21, 22

permutation, 75

pivot balancedness, see balancedness

pivotal player, see player

player, 13

$$
\begin{aligned}
& \text { apex, } 16, \mathbf{1 8}, 23 \\
& \text { controlled, } 83 \\
& \text { minor, } 16, \mathbf{1 8}, \mathbf{1 9}, 23,36 \\
& \text { null } \\
& \quad \text { in } S, \mathbf{1 7}, 22,26,33 \\
& \quad \text { with respect to } C, 64, \mathbf{7 5} \\
& \quad \text { with respect to } v, \mathbf{1 3} \\
& \text { pivotal, } \mathbf{1 3}, 16 \\
& \text { symmetric, } \mathbf{1 4} \\
& \text { uncontrolled, } 87
\end{aligned}
$$


veto

in $S, \mathbf{1 7}$

with respect to $v, \mathbf{1 4}$

power, 9

power configuration, 40, 41

efficient, 40

individually rational, $\mathbf{4 0}, 46,61$

stable, 41, 43, 47, 48, 51

stable under $\mathcal{R}, \mathbf{6 0}$

power index

on mutual control structures, 13 , $64,74, \mathbf{7 5}, 81$

anonymity, $64, \mathbf{7 5}, 81-83,85$

constant sum, $\mathbf{7 5}, 81-83,85$

controlled player condition, 83 ,

83

monotonicity, 65, 82, 82

null player property, $\mathbf{7 5}, 78,81-$ 84

transfer property, 64, 75, 76, 78,

81-83, 85

on simple games, $\mathbf{2 0}, 21$

efficient, 20, 22, 24

equal treatment, 20, 22-24, 28

null player property, 20, 23, 24, 28

strongly efficient, 24

strongly monotonic, 16, 20, 20, $22-24,32,33,36$

preference profile, 21 , see preferences

preferences, $9,11,12, \mathbf{2 1}, 37,39$

profile of, 21

proper simple game, see simple game

proportional bargaining solution, see bargaining solution
Shapley value, see Shapley-Shubik index

Shapley-Shubik index, 10, 16, 26, 36, $37,64,80$

share, 63,65

simple game, $9, \mathbf{1 3}, 64,80$

monotonic, 13, 64

proper, $\mathbf{1 3}$

proper monotonic, 13, 21, 26, 40, $41,43,47,48,51,52$

vector of, 70

associated mutual control structure, 72

extension, $\mathbf{7 3}$

invariant, $73, \mathbf{7 3}$

invariant extension, $73, \mathbf{7 3}$

minimal invariant extension, $\mathbf{7 3}$, 74

social choice, 9

subgame, 11, 17, 23

substitutable, 22

supervisory board, 18

symmetric player, see player

syndicate, 27

unanimity mutual control structure, see mutual control structure

veto player, see player

veto power, 10

weak top coalition property, 21

winner takes all, 87

winning coalition, see coalition

zero game, 13

quota

apex, 16, 19

minor, 16, 19

restriction, $\mathbf{1 7}, 22$ 



\section{Summary}

Each chapter of this dissertation is based on one article. The first two consider simple games and coalitions formation games while the third one introduces a new model of control, namely mutual control structures.

The first chapter is based on Karos (2012). It generalizes the class of apex games games with one apex player to a class of games with a collection of apex sets. These simple games, together with a power index, canonically induce a hedonic coalition formation game. We analyze a monotonicity property of power indices and its meaning for the induced hedonic game. In particular, we give necessary and sufficient conditions for the existence of core stable coalitions in this hedonic game. We also consider the special cases of Shapley-Shubik index, Banzhaf index, and normalized Banzhaf index. The first two are monotonic, while the latter is not. We show that in case of classical apex games with one apex player, there is no core stable coalition if we apply the Shapley-Shubik index or the normalized Banzhaf index.

The second chapter is based on Karos (2013). Here we introduce power configurations. In contrast to a power index a power configuration specifies the power of each player in each winning coalition. We introduce a new power configuration which takes into account bargaining among players in coalitions. We show that under very weak conditions on a bargaining solution there is a power configuration which is stable with respect to renegotiations. We further show that given this power configuration there is a coalition which is both internally and Nash stable. We consider two different bargaining solutions on apex games and show under which conditions there are core stable coalitions. Finally, we investigate how infeasible coalition might affect the outcome and apply our model to the German parliament.

The last chapter is based on Karos and Peters (2013) and introduces the notion of mutual control structures. In a mutual control structure agents exercise control over each other. Typical examples occur in the area of corporate governance: firms and investment companies exercise mutual control, in particular by owning each others' stocks. In this paper we formulate a general model for such situations. There is a fixed set of agents, and a mutual control structure assigns to each subset (coalition) the subset of agents controlled by that coalition. Such a mutual control structure captures direct control. We propose a procedure in order to incorporate indirect control as well: if $S$ controls $T$, and $S$ and $T$ jointly control $R$, then $S$ controls $R$ indirectly. This way, invariant mutual control structures result. Alternatively, mutual control can be described by vectors of simple games - simple game structures, each simple game describing who controls a certain player, and also those simple games can be updated in order to capture indirect control. We show that both approaches lead to equivalent 
invariant structures.

In the second part of the paper, we axiomatically develop a class of power indices for invariant mutual control structures. We impose four axioms with a plausible interpretation in this framework, which together characterize a broad class of power indices based on dividends resulting both from exercising and from undergoing control. By adding an extra condition a unique power index is singled out. In this index, each player accumulates his Shapley-Shubik power index assignments from controlling other players, diminished by the sum of the Shapley-Shubik power index assignments to other players controlling him.

\section{About the Author}

Dominik Karos was born on July 6, 1987 in Saarbrücken, Germany. He studied mathematics at Saarland University and reached his diploma in 2010. During the last two years of his studies he worked as consultant for financial risk management in Luxembourg. After his graduation he quit this position and started as research and teaching assistant at the Chair of Economic Theory at Saarland University under the supervision of Dinko Dimitrov in June 2010. From November 2011 he was under joint supervision of Dinko Dimitrov and Hans Peters, Maastricht University.

His research focuses on cooperative game theory. He is particularly interested in power indices and coalition formation. This dissertation is based on his research from June 2010 to May 2013. 